\title{
Turn-On Sulfide $\pi$ Donors: An Ultrafast Push for Twisted Mechanophores
}

Quentin Verolet, ${ }^{\dagger}$ Arnulf Rosspeintner, ${ }^{\dagger}$ Saeideh Soleimanpour, Naomi Sakai, Eric Vauthey,* and Stefan Matile*

School of Chemistry and Biochemistry, University of Geneva, CH-1211 Geneva, Switzerland

Supporting Information

Table of Content

1. Material and Methods

S2

2. Synthesis

S4

3. Solvatochromism

4. Femtosecond Time-Resolved Broadband Fluorescence Experiments

5. Supporting References

6. NMR Spectra

S26

7. MALDI-TOF MS

S45 


\section{Materials and Methods}

As in ref. S1, Supporting Information. Briefly, reagents for synthesis were purchased from Fluka, Sigma-Aldrich, Acros, and Apollo Scientific; buffers and salts of the best grade available from Fluka or Sigma-Aldrich were used as received. For the ultrafast measurements n-hexane (Roth, Rotisolv 99\% UV/IR grade), cyclohexane (Acros, ExtraDry 99.5\%), hexadecane (Sigma-Aldrich, Reagent Plus 99\%) and ethyl acetate (Acros, 99.5\% for spectroscopy) were used as received.

Unless stated otherwise, column chromatography was carried out on silica gel (SiliaFlash ${ }^{\circledR}$ P60, SILICYCLE, 230-400 mesh). Analytical TLC was performed on silica gel $60 \mathrm{~F}_{254}$ (Merck). UV-Vis spectra were recorded on a JASCO V-650 spectrophotometer equipped with a stirrer and a temperature controller and are reported as maximal absorption wavelength in $\mathrm{nm}$. Fluorescence measurements were performed with a FluoroMax-4 spectrofluorometer (Horiba Scientific) equipped with a stirrer and a temperature controller. Fluorescence spectra were corrected using instrument-supplied correction factors, unless stated otherwise. Melting points (Mp) were recorded on a Büchi Melting Point M-565. IR spectra were recorded on a Perkin Elmer Spectrum One FT-IR spectrometer (ATR, Golden Gate) and are reported as wavenumbers $v$ in $\mathrm{cm}^{-1}$ with band intensities indicated as s (strong), m (medium), w (weak), br (broad). ${ }^{1} \mathrm{H}$ and ${ }^{13} \mathrm{C}$ NMR spectra were recorded (as indicated) either on a Bruker $300 \mathrm{MHz}, 400 \mathrm{MHz}$ or $500 \mathrm{MHz}$ spectrometer and are reported as chemical shifts $(\delta)$ in ppm relative to (residual) solvent signal. Spin multiplicities are reported as a singlet (s), doublet (d), triplet (t), quartet (q), quintet (quint) or multiplet (m), with coupling constants $(J)$ given in $\mathrm{Hz} .{ }^{13} \mathrm{C}$ resonances were assigned with the aid of additional information from DEPT 135 spectra.

ESI-MS for the characterization of new compounds was performed on an ESI API 150EX and are reported as mass-per-charge ratio $\mathrm{m} / \mathrm{z}$ (intensity in \%, [assignment]). ESI-HRMS for 
the characterization of new compounds were performed on a QSTAR Pulsar (AB/MDS Sciex) and are reported as mass-per-charge ratio $\mathrm{m} / \mathrm{z}$ calculated and observed. MALDI-MS analysis were performed using Bruker MALDI Autoflex Speed TOF/TOF.

Abbreviations. $\mathrm{Ac}_{2} \mathrm{O}$ : Acetic anhydride; $\mathrm{ACN}$ : Acetonitrile; $\mathrm{AcOH}$ : Acetic acid; $n$ BuLi: $n$-Butyllithium; $\mathrm{Bu}_{3} \mathrm{SnCl}$ : Tributyltin chloride; Calcd: Calculated; DCM: Dichloromethane; DIPEA: Diisopropyl ethylamine; DMF: $N, N$-Dimethylformamide; DMSO: Dimethylsulfoxide; Et ${ }_{2} \mathrm{O}$ : Diethyl ether; EtOAc: Ethyl acetate; EtOH: Ethanol; LDA: Lithium diisopropyl amide; mCPBA: 3-Chloroperbenzoic acid; $\mathrm{MeOH}$ : Methanol; NBS: $N$ Bromosuccinimide; rt: Room temperature; PE: Petroleum ether; TfOH: Trifluoromethanesulfonic acid, THF: Tetrahydrofuran. 


\section{Synthesis}

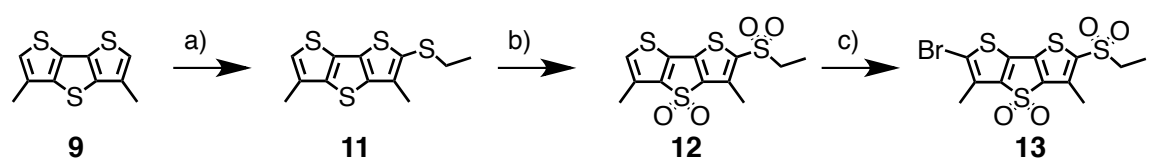

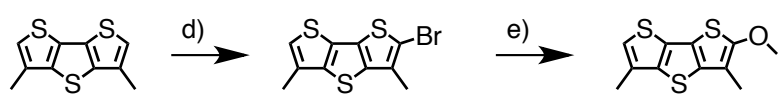

9

14

15

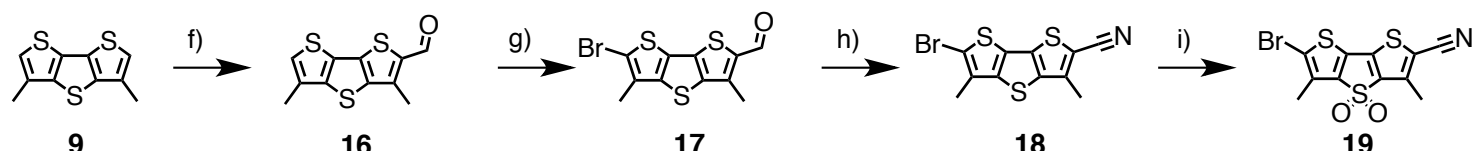

16

17

18

19
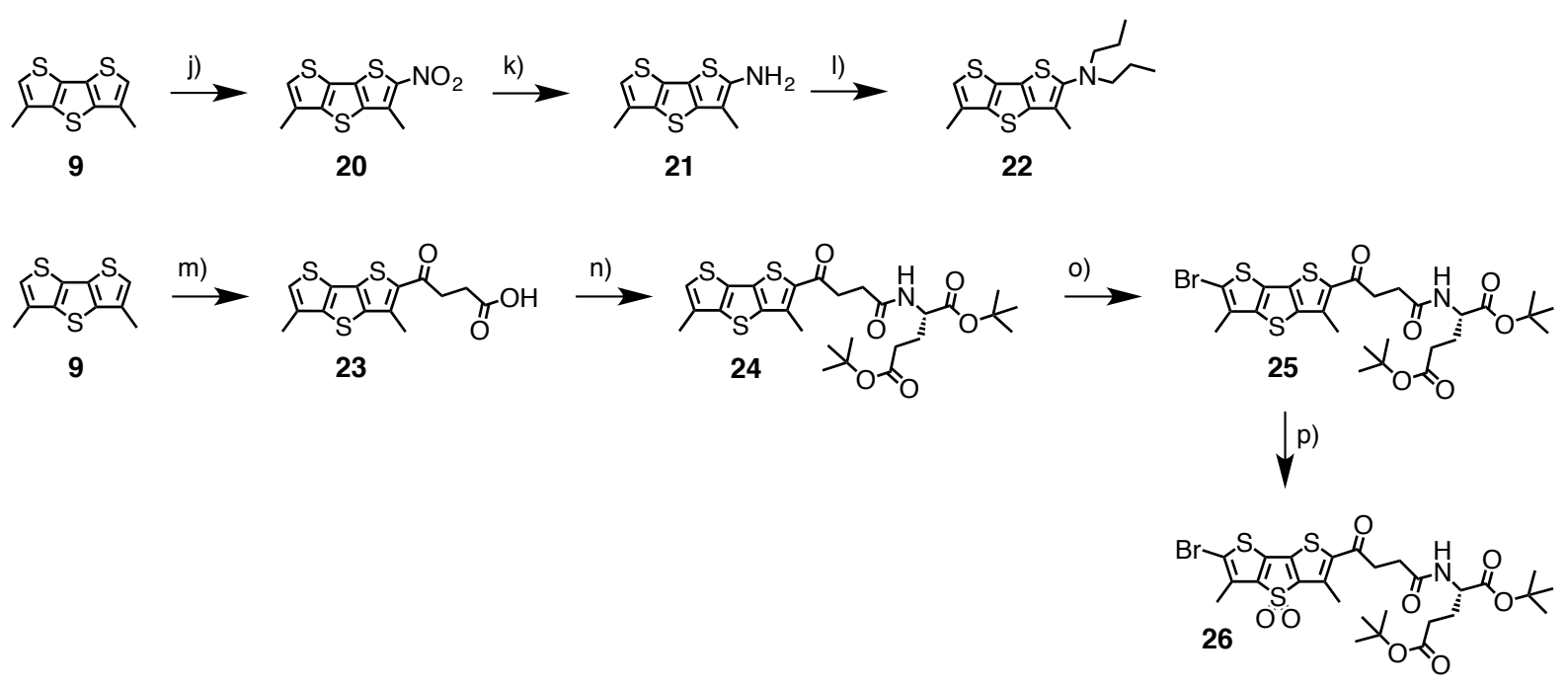

Scheme S1. Reagents and conditions: (a) 1. LDA, THF, $-78^{\circ} \mathrm{C}, 30 \mathrm{~min}$, 2. diethyldisulfide (10), THF, rt, 12 h, 70\%; (b) mCPBA, $\mathrm{CHCl}_{3}, \mathrm{rt}, 12$ h, 53\%; (c) NBS, AcOH, DCM, reflux, 24 h, 66\%; (d) NBS, DMF, $0{ }^{\circ} \mathrm{C}, 30 \mathrm{~min}, 70 \%$; (e) $\mathrm{CuBr}$, NaOMe, methyl formate, toluene, $100{ }^{\circ} \mathrm{C}, 2$ days, 52\%; (f) $\mathrm{POCl}_{3}$, DMF, $50{ }^{\circ} \mathrm{C}, 2 \mathrm{~h}, 75 \%$; (g) NBS, DMF, $80{ }^{\circ} \mathrm{C}, 24 \mathrm{~h}, 80 \%$; (h) $\mathrm{NaN}_{3}, \mathrm{TfOH}, \mathrm{ACN}$, reflux, 4 h, 70\%; (i) mCPBA, DCM, rt, 12 h, 65\%; (j) $\mathrm{Ac}_{2} \mathrm{O}, \mathrm{HNO}_{3}, 0$ ${ }^{\circ} \mathrm{C}, 30 \mathrm{~min}, 45 \%$; (k) $\mathrm{Pd} / \mathrm{C}, \mathrm{H}_{2}$, THF, rt, 5 h; (l) propanal, $\mathrm{NaCNBH}_{3}, \mathrm{AcOH}, \mathrm{THF}, \mathrm{rt}, 3 \mathrm{~h}$, 56\%; (m) $\mathrm{AlCl}_{3}$, succinic anhydride, DCM, rt, 12 h, 10\%; (n) HBTU, DIEPA, H-Glu(t-Bu)-tBu, DMF, rt, 2h, 60\%; (o) NBS, DCM, rt, 6 h, 60\%; (p) mCPBA, DCM, rt, 12 h, 73\%. 


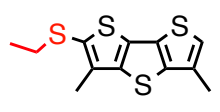

11

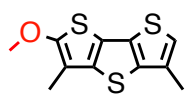

15

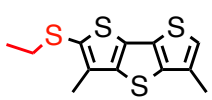

11<smiles>COc1sc2c(sc3c(C)csc32)c1C</smiles>

15

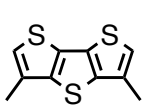

9<smiles></smiles>

22<smiles>CCCN(CCC)Cc1sc2c(c1C)Sc1sc(C)cc1S2</smiles>

22

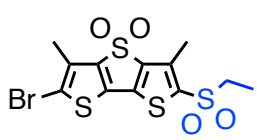

13

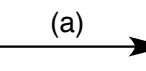

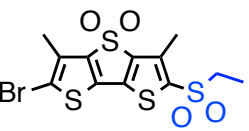

13

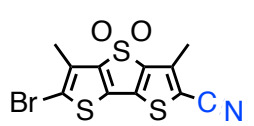

19

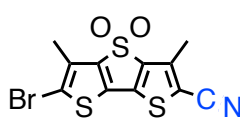

19

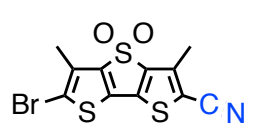

19

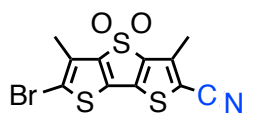

(a)

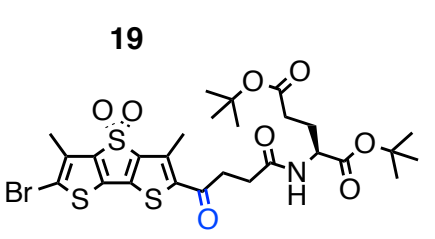

26

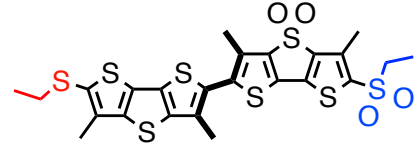

2
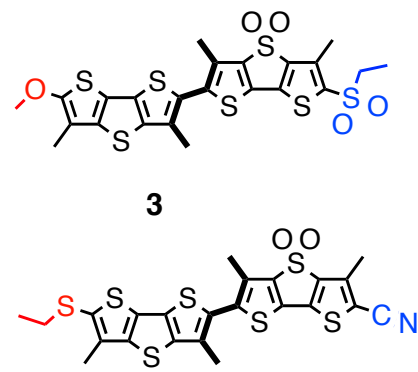

4

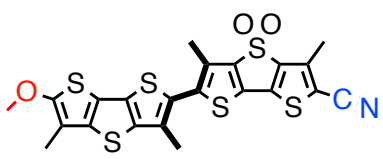

5

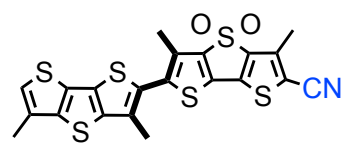

6

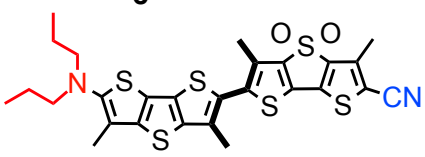

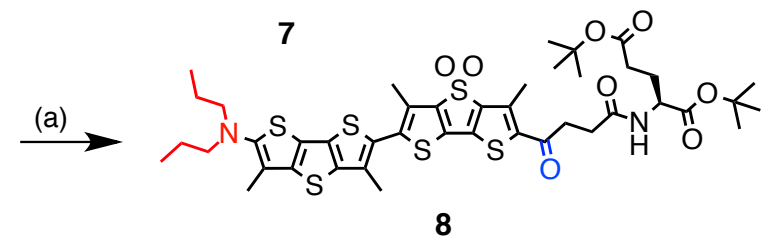

Scheme S2. Reagents and conditions: (a) 1. $\mathrm{LDA}, \mathrm{SnCl}(\mathrm{Bu})_{3}, \mathrm{THF},-78^{\circ} \mathrm{C}$ to $\mathrm{rt}, 1 \mathrm{~h}, 2$. $\mathrm{Pd}\left(\mathrm{PPh}_{3}\right)_{4}, \mathrm{DMF}, 70{ }^{\circ} \mathrm{C}, 48 \mathrm{~h}$. 
Compound 9 was synthesized according to the procedure reported in ref. S2.

Compound 14 was synthesized according to the procedure reported in ref. S3.

Compound 16 was synthesized according to the procedure reported in ref. S1.

Compound 17 was synthesized according to the procedure reported in ref. S1.

Compound 11. To a solution of $9(100 \mathrm{mg}, 0.446 \mathrm{mmol})$ in dry THF $(5 \mathrm{~mL})$ at $-78{ }^{\circ} \mathrm{C}$ under Ar atmosphere, LDA $(0.29 \mathrm{~mL}, 2 \mathrm{M}$ in THF/heptane/ethylbenzene, $0.58 \mathrm{mml})$ was added dropwise. After $30 \mathrm{~min}$, diethyl disulfide (10,80 $\mathrm{mg}, 0.65 \mathrm{mmol})$ was added, and the reaction mixture was allowed to warm to rt and stirred overnight. The crude was diluted with EtOAc $(20 \mathrm{~mL})$ and washed with water $(30 \mathrm{~mL})$ and brine $(30 \mathrm{~mL})$, then the organic phase was dried over $\mathrm{Na}_{2} \mathrm{SO}_{4}$. The crude was purified by flash chromatography $\left(\mathrm{SiO}_{2}, \mathrm{PE}\right)$ to afford compound 11 as a colorless oil (89 mg, 70\%). $R_{\mathrm{f}}(\mathrm{PE}): 0.41$; IR (neat): $2964(\mathrm{w}), 2917(\mathrm{~m})$, 2856 (w), 1436 (m), 1376 (s), 1360 (s), 1255 (m), 1099 (w), 1078 (m), 1025 (m), 997 (w), 970 (m), 914 (m), 829 (m), 730 (s); ${ }^{1} \mathrm{H}$ NMR (400 MHz, $\left.\mathrm{CD}_{2} \mathrm{Cl}_{2}\right): 7.00$ (q, ${ }^{4} J(\mathrm{H}, \mathrm{H})=1.2 \mathrm{~Hz}$, $1 \mathrm{H}), 2.81\left(\mathrm{q},{ }^{3} J(\mathrm{H}, \mathrm{H})=7.4 \mathrm{~Hz}, 3 \mathrm{H}\right), 2.43(\mathrm{~s}, 3 \mathrm{H}), 2.38\left(\mathrm{~d},{ }^{4} J(\mathrm{H}, \mathrm{H})=1.2 \mathrm{~Hz}, 3 \mathrm{H}\right), 1.26\left(\mathrm{t},{ }^{3} J\right.$ $(\mathrm{H}, \mathrm{H})=7.4 \mathrm{~Hz}, 3 \mathrm{H}) ;{ }^{13} \mathrm{C}$ NMR (101 MHz, $\left.\mathrm{CD}_{2} \mathrm{Cl}_{2}\right): 142.6(\mathrm{C}), 142.4(\mathrm{C}), 137.2(\mathrm{C}), 131.7$ (C), $131.6(\mathrm{C}), 131.1(\mathrm{C}), 129.2(\mathrm{C}), 122.1(\mathrm{CH}), 33.6\left(\mathrm{CH}_{2}\right), 15.2\left(\mathrm{CH}_{3}\right), 14.9\left(\mathrm{CH}_{3}\right), 14.1$ $\left(\mathrm{CH}_{3}\right) ; \mathrm{MS}(\mathrm{ESI}+, \mathrm{MeOH}): 568\left(100,[2 \mathrm{M}+\mathrm{H}]^{+}\right), 285\left(80,[\mathrm{M}+\mathrm{H}]^{+}\right)$.

Compound 12. To a solution of $11(500 \mathrm{mg}, 1.76 \mathrm{mmol})$ in $\mathrm{CHCl}_{3}(30 \mathrm{~mL}), \mathrm{mCPBA}$ $(70 \%, 2.17 \mathrm{~g}, 8.79 \mathrm{mmol})$ was added in several portions and the mixture was stirred overnight at $\mathrm{rt}$. The reaction was diluted with DCM $(30 \mathrm{~mL})$ and washed with a saturated solution of $\mathrm{NaHCO}_{3}(3 \times 30 \mathrm{~mL})$ and the aqueous layers were extracted with DCM $(3 \times 30 \mathrm{~mL})$. The combined organic phases were washed with $\mathrm{H}_{2} \mathrm{O}(20 \mathrm{~mL})$ and brine $(20 \mathrm{~mL})$ and finally dried over $\mathrm{Na}_{2} \mathrm{SO}_{4}$. The crude was purified by flash chromatography $\left(\mathrm{SiO}_{2}, \mathrm{DCM}\right)$ to afford 
compound 12 as a yellow solid (325 mg, 53\%). $R_{\mathrm{f}}(\mathrm{DCM}): 0.19$; Mp: decomposition $>200$ ${ }^{\circ} \mathrm{C}$; IR (neat): 2924 (w), 2856 (w), 1412 (w), 1302 (s), 1157 (m), 1130 (s), 1072 (m), 1043 (m), 986 (m), 841 (w), 743 (m), 718 (s), 6648 (s), 539 (s); ${ }^{1} \mathrm{H}$ NMR (500 MHz, $\mathrm{CD}_{2} \mathrm{Cl}_{2}$ ): 7.13 $\left(\mathrm{q},{ }^{4} J(\mathrm{H}, \mathrm{H})=1.1 \mathrm{~Hz}, 1 \mathrm{H}\right), 3.23\left(\mathrm{q},{ }^{3} J(\mathrm{H}, \mathrm{H})=7.4 \mathrm{~Hz}, 2 \mathrm{H}\right), 2.61(\mathrm{~s}, 3 \mathrm{H}), 2.40\left(\mathrm{~d},{ }^{4} J(\mathrm{H}, \mathrm{H})=\right.$ $1.1 \mathrm{~Hz}, 3 \mathrm{H}), 1.34\left(\mathrm{t},{ }^{3} J(\mathrm{H}, \mathrm{H})=7.4 \mathrm{~Hz}, 3 \mathrm{H}\right) ;{ }^{13} \mathrm{C} \mathrm{NMR}\left(126 \mathrm{MHz}, \mathrm{CD}_{2} \mathrm{Cl}_{2}\right): 144.4(\mathrm{C}), 143.9$ (C), $140.6(\mathrm{C}), 138.9(\mathrm{C}), 136.9(\mathrm{C}), 134.3(\mathrm{C}), 133.8(\mathrm{C}), 129.0(\mathrm{CH}), 52.4\left(\mathrm{CH}_{2}\right), 13.3$ $\left(\mathrm{CH}_{3}\right), 12.5\left(\mathrm{CH}_{3}\right), 8.0\left(\mathrm{CH}_{3}\right) ; \mathrm{MS}(\mathrm{ESI}+, \mathrm{ACN}): 1062\left(16,\left[3 \mathrm{M}+\mathrm{NH}_{4}\right]^{+}\right), 714(75$, $\left.\left[2 \mathrm{M}+\mathrm{NH}_{4}\right]^{+}\right), 366\left(100,\left[\mathrm{M}+\mathrm{NH}_{4}\right]^{+}\right)$; HRMS (ESI, +ve) calcd for $\mathrm{C}_{12} \mathrm{H}_{12} \mathrm{O}_{4} \mathrm{~S}_{4}: 348.9691$, found: 348.9691 .

Compound 13. To a solution of $12(325 \mathrm{mg}, 0.933 \mathrm{mmol})$ in DCM/AcOH (10:5 mL), NBS (200 mg, $1.19 \mathrm{mmol}$ ) was added. The reaction mixture was stirred at reflux for $24 \mathrm{~h}$. The solution was cooled to rt, washed with water $(3 \times 30 \mathrm{~mL})$ and the organic phase was dried over $\mathrm{Na}_{2} \mathrm{SO}_{4}$. The crude was purified by flash chromatography $\left(\mathrm{SiO}_{2}\right.$, toluene/EtOAc 10:0.5) to afford compound 13 as a yellow solid (263 mg, 66\%). $R_{\mathrm{f}}$ (toluene/EtOAc 10:0.5): 0.22; Mp: decomposition $>200{ }^{\circ} \mathrm{C}$; IR (neat): 1503 (w), 1450 (w), 1411 (m), 1308 (s), 1159 (m), 1132 (s), $1114(\mathrm{~m}), 1078$ (m), 1044 (m), 994 (m), 951 (w), 789 (m), 726 (s), 639 (s), 541 (s), $543(\mathrm{~m}) ;{ }^{1} \mathrm{H}$ NMR $\left(500 \mathrm{MHz}, \mathrm{CD}_{2} \mathrm{Cl}_{2}\right): 3.24\left(\mathrm{q},{ }^{3} J(\mathrm{H}, \mathrm{H})=7.4 \mathrm{~Hz}, 2 \mathrm{H}\right), 2.63(\mathrm{~s}, 3 \mathrm{H}), 2.35(\mathrm{~s}$, $3 \mathrm{H}), 1.36\left(\mathrm{t},{ }^{3} J(\mathrm{H}, \mathrm{H})=7.4 \mathrm{~Hz}, 3 \mathrm{H}\right) ;{ }^{13} \mathrm{C} \mathrm{NMR}\left(126 \mathrm{MHz}, \mathrm{CD}_{2} \mathrm{Cl}_{2}\right): 143.2(\mathrm{C}), 142.7(\mathrm{C})$, $140.0(\mathrm{C}), 138.9$ (C), $137.4(\mathrm{C}), 133.5(\mathrm{C}), 132.7(\mathrm{C}), 117.8(\mathrm{C}), 52.5\left(\mathrm{CH}_{2}\right), 12.9\left(\mathrm{CH}_{3}\right), 12.5$ $\left(\mathrm{CH}_{3}\right), 8.0\left(\mathrm{CH}_{3}\right)$; MS (ESI+, ACN, $n / n$ : isotopic peaks): 870/872 (50, [2M+NH$\left.]^{+}\right), 444 / 446$ $\left(100,\left[\mathrm{M}+\mathrm{NH}_{4}\right]^{+}\right)$; HRMS (ESI, + ve) calcd for $\mathrm{C}_{12} \mathrm{H}_{11} \mathrm{O}_{4} \mathrm{~S}_{4} \mathrm{BrNa}$ : 448.8616, found: 448.8634 .

Compound 15. In a Schlenk tube under Ar atmosphere, 14 (290 mg, $0.956 \mathrm{mmol})$ was dissolved in dry degassed toluene $(30 \mathrm{~mL})$. To this solution $\mathrm{CuBr}(68 \mathrm{mg}, 0.47 \mathrm{mmol})$, $\mathrm{NaOMe}(2.6 \mathrm{~mL}, 5.4 \mathrm{M}$ in $\mathrm{MeOH}, 14 \mathrm{mmol})$, and methyl formate $(0.05 \mathrm{~mL}, 0.8 \mathrm{mmol})$ were added. The reaction mixture was heated at $100{ }^{\circ} \mathrm{C}$ for two days. The crude mixture was 
diluted with DCM (200 mL) and washed with water $(3 \times 100 \mathrm{~mL})$ and the organic phase was dried over $\mathrm{Na}_{2} \mathrm{SO}_{4}$. Flash chromatography $\left(\mathrm{SiO}_{2}, \mathrm{PE}\right)$ afforded compound $\mathbf{1 5}$ as a white solid (128 mg, 52\%). $R_{\mathrm{f}}(\mathrm{PE}): 0.15 ; \mathrm{Mp}: 116-118^{\circ} \mathrm{C}$; IR (neat): $2921(\mathrm{~m}), 2853(\mathrm{w}), 1562(\mathrm{~m})$, 1455 (m), 1402 (w), $1365(\mathrm{w}), 1237$ (m), $1171(\mathrm{w}), 1059$ (m), $999(\mathrm{~m}), 946(\mathrm{~m}), 824(\mathrm{w}), 736$ (m); ${ }^{1} \mathrm{H}$ NMR $\left(500 \mathrm{MHz}, \mathrm{CD}_{2} \mathrm{Cl}_{2}\right): 6.89\left(\mathrm{q},{ }^{4} J(\mathrm{H}, \mathrm{H})=1.1 \mathrm{~Hz}, 1 \mathrm{H}\right), 3.95(\mathrm{~s}, 3 \mathrm{H}), 2.36\left(\mathrm{~d},{ }^{4} J\right.$ $(\mathrm{H}, \mathrm{H})=1.1 \mathrm{~Hz}, 3 \mathrm{H}), 2.20(\mathrm{~s}, 3 \mathrm{H}) ;{ }^{13} \mathrm{C} \mathrm{NMR}\left(126 \mathrm{MHz}, \mathrm{CD}_{2} \mathrm{Cl}_{2}\right): 159.5(\mathrm{C}), 140.3(\mathrm{C}), 139.6$ (C), $131.9(\mathrm{C}), 131.5(\mathrm{C}), 119.9(\mathrm{C}), 118.4(\mathrm{C}), 113.4(\mathrm{C}), 63.4\left(\mathrm{CH}_{3}\right), 15.0\left(\mathrm{CH}_{3}\right), 10.8$ $\left(\mathrm{CH}_{3}\right) ; \mathrm{MS}(\mathrm{ESI}+\mathrm{MeOH}): 255\left(100,[\mathrm{M}+\mathrm{H}]^{+}\right)$.

Compound 18. A solution of $17(500 \mathrm{mg}, 1.54 \mathrm{mmol})$ in dry ACN (75 mL) was obtained by heating at $90{ }^{\circ} \mathrm{C}$ under $\mathrm{Ar}$ atmosphere. To this solution were added $\mathrm{NaN}_{3}$ (235 mg, 3.62 mmol) and TfOH $(0.67 \mathrm{~mL}, 7.6 \mathrm{mmol})$. The reaction mixture was stirred at reflux for $4 \mathrm{~h}$. The crude was diluted in DCM $(100 \mathrm{~mL})$ and washed with water $(30 \mathrm{~mL})$ and brine $(30 \mathrm{~mL})$, and the aqueous layers were extracted with DCM $(3 \times 30 \mathrm{~mL})$. The combined organic phases were dried over $\mathrm{Na}_{2} \mathrm{SO}_{4}$, filtered and concentrated under vacuum to obtain a dark blue residue. Product was purified by flash chromatography $\left(\mathrm{SiO}_{2}, \mathrm{DCM} / \mathrm{PE} \mathrm{1:1)}\right.$ to afford compound $\mathbf{1 8}$ as a colorless solid (346 mg, 70\%). $R_{\mathrm{f}}(\mathrm{DCM} / \mathrm{PE} 1: 1): 0.3$; Mp: $180-181{ }^{\circ} \mathrm{C}$; IR (neat): 3106 (w), 2923 (w), 2213 (m), 1508 (w), 1414 (m), 1297 (s), 1165 (s), 1092 (w), 843 (m), 766 (s), 665 (m); ${ }^{1} \mathrm{H}$ NMR (400 MHz, $\left.\mathrm{CDCl}_{3}\right): 2.57$ (s, 3H), $2.34(\mathrm{~s}, 3 \mathrm{H}) ;{ }^{13} \mathrm{C}$ NMR $(100 \mathrm{MHz}$, $\left.\mathrm{CDCl}_{3}\right): 144.8(\mathrm{C}), 143.0(\mathrm{C}), 140.1$ (C), 134.1 (C), 131.3 (C), 128.6 (C), 114.7 (C), 113.0 (C), $105.0(\mathrm{C}), 15.0\left(\mathrm{CH}_{3}\right), 14.6\left(\mathrm{CH}_{3}\right)$; $\mathrm{MS}(\mathrm{ESI}+, \mathrm{MeOH}, n / n$ : isotopic peaks): 327/329 $\left(100,[\mathrm{M}+\mathrm{H}]^{+}\right)$.

Compound 19. To a solution of 18 (346 mg, $1.06 \mathrm{mmol})$ in DCM (20 mL), mCPBA (519 $\mathrm{mg}, 2.11 \mathrm{mmol}$ ) was added in several portions and the mixture was stirred overnight at $\mathrm{rt}$. A saturated solution of $\mathrm{NaHCO}_{3}(30 \mathrm{~mL})$ was added and layers were separated. The aqueous layer was extracted with DCM (3 x $30 \mathrm{~mL})$, and the combined organic phase was collected 
and washed with a saturated solution of $\mathrm{NaHCO}_{3}(3 \times 10 \mathrm{~mL})$. The organic phase was dried over $\mathrm{Na}_{2} \mathrm{SO}_{4}$. The crude was purified by flash chromatography $\left(\mathrm{SiO}_{2}, \mathrm{DCM}\right)$ to afford compound 19 as a yellow solid (248 mg, 65\%). $R_{\mathrm{f}}(\mathrm{DCM}): 0.2 ; \mathrm{Mp}$ : decomposition $>200{ }^{\circ} \mathrm{C}$; IR (neat): 2923 (w), 2206 (m), 1504 (w), 1451 (m), 1407 (m), 1380 (s), 1305 (s), 1289 (s), 1144 (s), 1097 (m), 1029 (w), 802 (w), 627 (m); ${ }^{1} \mathrm{H}$ NMR (400 MHz, $\mathrm{CDCl}_{3}$ ): 2.59 (s, 3H), 2.37 (s, 3H); ${ }^{13} \mathrm{C}$ NMR (100 MHz, $\left.\mathrm{CDCl}_{3}\right): 143.9$ (C), 143.8 (C), 140.9 (C), 139.6 (C), 133.4 (C), $131.9(\mathrm{C}), 117.5(\mathrm{C}), 112.5(\mathrm{C}), 109.1(\mathrm{C}), 13.2\left(\mathrm{CH}_{3}\right), 12.8\left(\mathrm{CH}_{3}\right)$; MALDI-TOF (DCTB): $332\left(100,[\mathrm{M}]^{+}\right)$.

Compound 20. To a solution of $9(500 \mathrm{mg}, 2.23 \mathrm{mmol})$ in $\mathrm{Ac}_{2} \mathrm{O}(2 \mathrm{~mL})$, a solution of $\mathrm{HNO}_{3}(0.3 \mathrm{~mL}, 6 \mathrm{mmol}, 65 \%)$ in $\mathrm{Ac}_{2} \mathrm{O}(2 \mathrm{~mL})$ was added dropwise at $0{ }^{\circ} \mathrm{C}$. The reaction mixture was kept at $0{ }^{\circ} \mathrm{C}$ for $30 \mathrm{~min}$. The mixture was poured into ice and water $(200 \mathrm{~mL})$ and stirred for additional $45 \mathrm{~min}$. The mixture was extracted with DCM (3 x $20 \mathrm{~mL})$ and organic layers were separated and dried over $\mathrm{Na}_{2} \mathrm{SO}_{4}$, filtered and concentrated under vacuum to obtain a brown residue. Product was purified by flash chromatography $\left(\mathrm{SiO}_{2}, \mathrm{DCM} / \mathrm{PE}\right.$ 1:2) to afford compound 20 as a yellow solid (361 mg, 45\%). $R_{\mathrm{f}}(\mathrm{DCM} / \mathrm{PE}$ 9:1): 0.2; Mp: 162 - $163{ }^{\circ} \mathrm{C}$; IR (neat): 2921 (w), 2313 (w), 2216 (w), 2024 (w), 1925 (w), 1503 (m), 1452 (m), 1373 (m), 1313 (s), 1283 (s), 1172 (m), 1028 (w), 827 (w), 774 (m), 742 (m), 637 (w); ${ }^{1} \mathrm{H}$ NMR (400 MHz, $\left.\mathrm{CD}_{2} \mathrm{Cl}_{2}\right): 7.28\left(\mathrm{q},{ }^{4} J(\mathrm{H}, \mathrm{H})=1.1 \mathrm{~Hz}, 1 \mathrm{H}\right), 2.79(\mathrm{~s}, 3 \mathrm{H}), 2.41\left(\mathrm{~d},{ }^{4} J(\mathrm{H}, \mathrm{H})=\right.$ $1.1 \mathrm{~Hz}, 3 \mathrm{H}) ;{ }^{13} \mathrm{C}$ NMR (100 MHz, $\left.\mathrm{CD}_{2} \mathrm{Cl}_{2}\right): 147.0(\mathrm{C}), 142.0$ (C), 136.8 (C), 132.7 (C), 131.7 (C), $130.1(\mathrm{C}), 126.4(\mathrm{C}), 100.3(\mathrm{CH}), 15.8\left(\mathrm{CH}_{3}\right), 14.7\left(\mathrm{CH}_{3}\right)$; MS (ESI+, MeOH): $271(50$, $\left.[\mathrm{M}+\mathrm{H}]^{+}\right), 224\left(100,\left[\mathrm{M}-\mathrm{NO}_{2}+\mathrm{H}\right]^{+}\right)$; HRMS (ESI, +ve) calcd for $\mathrm{C}_{10} \mathrm{H}_{7} \mathrm{NO}_{2} \mathrm{~S}_{3}: 268.9712$, found: 268.9714 .

Compound 22. To a solution of $20(200 \mathrm{mg}, 0.743 \mathrm{mmol})$ in dry THF $(10 \mathrm{~mL})$ under $\mathrm{N}_{2}$ atmosphere, $\mathrm{Pd} / \mathrm{C}(20 \mathrm{mg}, 10 \%)$ was added. The reaction mixture was bubbled with $\mathrm{H}_{2}$ for $5 \mathrm{~h}$ 
to afford compound 21. $R_{\mathrm{f}}(\mathrm{PE})$ : 0.2. The reaction mixture was bubbled with Ar for $5 \mathrm{~min}$ and let under Ar atmosphere. Propanal (215 mg, $3.71 \mathrm{mmol}), \mathrm{NaCNBH}_{3}(233 \mathrm{mg}, 3.71 \mathrm{mmol})$ and AcOH $(0.1 \mathrm{~mL}, 2 \mathrm{mmol})$ were added. The reaction was stirred for $3 \mathrm{~h}$, and then filtered through Celite. The filtrate was diluted with DCM $(30 \mathrm{~mL})$ and washed with a saturated aqueous solution of $\mathrm{NaHCO}_{3}(3 \times 30 \mathrm{~mL})$. The organic phase was dried over $\mathrm{Na}_{2} \mathrm{SO}_{4}$ and absorbed on silica. The product was purified by flash chromatography $\left(\mathrm{SiO}_{2}, \mathrm{PE}\right)$ to afford compound 22 as a colorless oil (136 mg, 56\%). $R_{\mathrm{f}}(\mathrm{PE}): 0.7$; IR (neat): 2957 (s), 2855 (s) 1552 (w), 1457 (m), $1379(\mathrm{~m}), 1265$ (w), $1212(\mathrm{~m}), 1140(\mathrm{w}), 1077$ (m) 997 (w), 964 (m), 887 (m), $830(\mathrm{w}), 722(\mathrm{~m}) ;{ }^{1} \mathrm{H}$ NMR $\left(400 \mathrm{MHz}, \mathrm{CD}_{2} \mathrm{Cl}_{2}\right): 6.92\left(\mathrm{q},{ }^{4} \mathrm{~J}(\mathrm{H}, \mathrm{H})=1.2 \mathrm{~Hz}, 1 \mathrm{H}\right), 2.92-$ $2.89(\mathrm{~m}, 4 \mathrm{H}), 2.38\left(\mathrm{~d},{ }^{4} J(\mathrm{H}, \mathrm{H})=1.2 \mathrm{~Hz}, 3 \mathrm{H}\right), 2.25(\mathrm{~s}, 3 \mathrm{H}), 1.54-1.45(\mathrm{~m}, 4 \mathrm{H}), 0.91\left(\mathrm{t},{ }^{3} J\right.$ $(\mathrm{H}, \mathrm{H})=7.4 \mathrm{~Hz}, 6 \mathrm{H}) ;{ }^{13} \mathrm{C}$ NMR $\left(100 \mathrm{MHz}, \mathrm{CD}_{2} \mathrm{Cl}_{2}\right): 153.3(\mathrm{C}), 140.8(\mathrm{C}), 140.5(\mathrm{C}), 132.4$ (C), $131.5(\mathrm{C}), 124.9(\mathrm{C}), 124.8(\mathrm{C}), 120.3(\mathrm{CH}), 60.6\left(2 \times \mathrm{CH}_{2}\right), 21.8\left(2 \mathrm{x} \mathrm{CH}_{2}\right), 15.0\left(\mathrm{CH}_{3}\right)$, $13.0\left(\mathrm{CH}_{3}\right), 12.0\left(2 \times \mathrm{CH}_{3}\right) ; \mathrm{MS}(\mathrm{ESI}+, \mathrm{MeOH}): 324\left(50,[\mathrm{M}+\mathrm{H}]^{+}\right), 282\left(100,\left[\mathrm{M}-\mathrm{C}_{3} \mathrm{H}_{7}+\mathrm{H}\right]^{+}\right)$.

Compound 23. To a solution of $9(500 \mathrm{mg}, 2.22 \mathrm{mmol})$ in dry DCM (20 mL) under Ar atmosphere, a solution of $\mathrm{AlCl}_{3}(445 \mathrm{mg}, 3.34 \mathrm{mmol})$ and succinic anhydride (290 mg, 2.89 mmol) was added dropwise at $0{ }^{\circ} \mathrm{C}$. After 10 min the mixture was allowed to come back to rt and stirred overnight. The crude was diluted with DCM $(100 \mathrm{~mL})$ and washed with $\mathrm{HCl}(100$ $\mathrm{mL}, 1 \mathrm{M})$. The aqueous phase was extracted twice with DCM $(100 \mathrm{~mL})$. The combined organic phase was dried over $\mathrm{Na}_{2} \mathrm{SO}_{4}$ and absorbed on silica. The product was purified by flash chromatography $\left(\mathrm{SiO}_{2}, \mathrm{DCM} / \mathrm{PE} / \mathrm{AcOH}\right.$ 9:1:0.1) to afford compound 23 as a yellow solid (75 mg, 10\%). $R_{\mathrm{f}}\left(\mathrm{DCM} / \mathrm{PE} / \mathrm{AcOH}\right.$ 9:1:0.1): 0.24; Mp: decomposes $>200{ }^{\circ} \mathrm{C}$; IR (neat): 2919 (w), 1702 (s), 1619 (s), 1496 (w), 1431 (m), 1391 (m), 1383 (m), 1356 (m), 1245 (m), 1212 (m), 1169 (m), 1060 (w), 932 (w), 883 (w); ${ }^{1} \mathrm{H}$ NMR (400 MHz, DMSO-d $): 12.19$ (s, 1H), $7.55-5.76(\mathrm{~m}, 1 \mathrm{H}), 3.19-3.16(\mathrm{~m}, 2 \mathrm{H}), 2.67(\mathrm{~s}, 3 \mathrm{H}), 2.61-2.58(\mathrm{~m}, 2 \mathrm{H}), 2.37\left(\mathrm{~d},{ }^{4} J\right.$ $(\mathrm{H}, \mathrm{H})=1.1 \mathrm{~Hz}, 3 \mathrm{H}) ;{ }^{13} \mathrm{C}$ NMR $\left(100 \mathrm{MHz}, \mathrm{DMSO}-d_{6}\right): 192.1(\mathrm{C}), 173.7$ (C), $144.6(\mathrm{C}), 143.7$ 
(C), 136.9 (C), 136.3 (C), 132.8 (C), 130.7 (C), 129.7 (C), $126.0(\mathrm{CH}), 35.7\left(\mathrm{CH}_{2}\right), 28.0$ $\left(\mathrm{CH}_{2}\right), 15.7\left(\mathrm{CH}_{3}\right), 14.1\left(\mathrm{CH}_{3}\right)$; MS (ESI+, MeOH): $326\left(100,[\mathrm{M}+\mathrm{H}]^{+}\right)$; HRMS (ESI, +ve) calcd for $\mathrm{C}_{14} \mathrm{H}_{13} \mathrm{O}_{3} \mathrm{~S}_{3}: 325.0021$, found: 325.0023 .

Compound 24. HBTU (263 mg, $0.693 \mathrm{mmol})$ and DIPEA $(0.28 \mathrm{~mL}, 1.6 \mathrm{mmol})$ were sequentially added to a solution of $\mathbf{2 3}(75 \mathrm{mg}, 0.23 \mathrm{mmol})$ in dry DMF $(10 \mathrm{~mL})$ under $\mathrm{N}_{2}$. The reaction mixture was stirred for $30 \mathrm{sec}$ and a solution of $\mathrm{H}-\mathrm{Glu}(t-\mathrm{Bu})-t-\mathrm{Bu}(205 \mathrm{mg}, 0.693$ mmol) in dry DMF (6 mL) was added dropwise. The reaction mixture was stirred at $\mathrm{rt}$ for $2 \mathrm{~h}$ and diluted with EtOAc $(80 \mathrm{~mL})$. The mixture was washed with $\mathrm{LiCl}(5 \%, 5 \times 50 \mathrm{~mL})$. The organic phase was dried over $\mathrm{Na}_{2} \mathrm{SO}_{4}$ and absorbed on silica. The product was purified by flash chromatography $\left(\mathrm{SiO}_{2}, \mathrm{PE} /\right.$ acetone $\left.10: 1.4\right)$ to afford compound 24 as a yellow solid (79 $\mathrm{mg}, 60 \%) . R_{\mathrm{f}}\left(\mathrm{PE} /\right.$ acetone 10:1.4): 0.22; Mp: $109-110{ }^{\circ} \mathrm{C}$; IR (neat): $3343(\mathrm{w}), 2977(\mathrm{w})$, $2927(\mathrm{w}), 1727$ (m), $1713(\mathrm{~m}), 1671$ (m), 1655 (m), 1527 (m), $1496(\mathrm{w}), 1451(\mathrm{w}), 1388(\mathrm{~m})$, 1367 (m), 1243 (m), 1204 (m), 1148 (s), 1073 (w), 845 (m), 788 (w); ${ }^{1} \mathrm{H}$ NMR (400 MHz, $\left.\mathrm{CD}_{2} \mathrm{Cl}_{2}\right): 7.15-7.14(\mathrm{~m}, 1 \mathrm{H}), 6.39-6.32(\mathrm{~m}, 1 \mathrm{H}), 4.47-4.41(\mathrm{~m}, 1 \mathrm{H}), 3.23-3.20(\mathrm{~m}, 2 \mathrm{H})$, $2.67(\mathrm{~s}, 3 \mathrm{H}), 2.66-2.62(\mathrm{~m}, 2 \mathrm{H}), 2.38\left(\mathrm{~d},{ }^{4} \mathrm{~J}(\mathrm{H}, \mathrm{H})=1.2 \mathrm{~Hz}, 3 \mathrm{H}\right), 2.34-2.26(\mathrm{~m}, 2 \mathrm{H}), 2.14-$ $2.05(\mathrm{~m}, 1 \mathrm{H}), 1.95-1.87(\mathrm{~m}, 1 \mathrm{H}), 1.47(\mathrm{~s}, 9 \mathrm{H}), 1.43(\mathrm{~s}, 9 \mathrm{H}) ;{ }^{13} \mathrm{C} \mathrm{NMR}\left(100 \mathrm{MHz}, \mathrm{CD}_{2} \mathrm{Cl}_{2}\right)$ : 192.8 (C), 172.7 (C), 171.9 (C), 171.7 (C), 145.9 (C), 145.2 (C), 138.5 (C), 136.0 (C), 133.9 (C), $131.9(\mathrm{C}), 130.7(\mathrm{C}), 124.7(\mathrm{CH}), 82.5(\mathrm{C}), 80.9(\mathrm{C}), 52.9(\mathrm{CH}), 36.8\left(\mathrm{CH}_{2}\right), 31.9\left(\mathrm{CH}_{2}\right)$, 30.6 $\left(\mathrm{CH}_{2}\right), 28.4\left(3 \times \mathrm{CH}_{3}\right), 28.35\left(\mathrm{CH}_{2}\right), 28.3\left(3 \times \mathrm{CH}_{3}\right), 16.3\left(\mathrm{CH}_{3}\right), 14.8\left(\mathrm{CH}_{3}\right)$; MS (ESI+, MeOH): $1132\left(16,[2 \mathrm{M}+\mathrm{H}]^{+}\right), 567\left(100,[\mathrm{M}+\mathrm{H}]^{+}\right)$; HRMS (ESI, +ve) calcd for $\mathrm{C}_{27} \mathrm{H}_{36} \mathrm{NO}_{6} \mathrm{~S}_{3}$ : 566.1699, found: 566.1686 .

Compound 25. To a solution of 24 (206 mg, $0.364 \mathrm{mmol})$ in DCM (10 mL), NBS (78 $\mathrm{mg}, 0.44 \mathrm{mmol}$ ) was added. The resulting solution was stirred at $\mathrm{rt}$ for $6 \mathrm{~h}$ in the dark. The reaction mixture was washed with water $(3 \times 30 \mathrm{~mL})$ and the organic phase was dried over $\mathrm{Na}_{2} \mathrm{SO}_{4}$. The crude was purified by flash chromatography $\left(\mathrm{SiO}_{2}, \mathrm{PE} /\right.$ acetone $\left.10: 0.25\right)$ to 
afford compound 25 as a yellow solid (152 mg, 60\%). $R_{\mathrm{f}}(\mathrm{PE} /$ acetone 10:0.25): 0.27; Mp: 124 - $125{ }^{\circ} \mathrm{C}$; IR (neat): 3315 (w), 2978 (w), 2927 (w), 1727 (s), 1653 (m), 1641 (m), 1534 (w), 1494 (w), 1450 (w), 1367 (m), 1251 (m), 1149 (s), 979 (w), 847 (m), 751 (w); ${ }^{1}$ H NMR (400 $\left.\mathrm{MHz}, \mathrm{CD}_{2} \mathrm{Cl}_{2}\right): 6.34\left(\mathrm{~d},{ }^{3} J(\mathrm{H}, \mathrm{H})=7.9 \mathrm{~Hz}, 1 \mathrm{H}\right), 4.43\left(\mathrm{td},{ }^{3} J(\mathrm{H}, \mathrm{H})=7.9,5.0 \mathrm{~Hz}, 1 \mathrm{H}\right), 3.28-$ $2.15(\mathrm{~m}, 2 \mathrm{H}), 2.68(\mathrm{~s}, 3 \mathrm{H}), 2.66-2.62(\mathrm{~m}, 2 \mathrm{H}), 2.32(\mathrm{~s}, 3 \mathrm{H}), 2.31-2.17(\mathrm{~m}, 2 \mathrm{H}), 2.13-2.05$ (m, 1H), 1.93 - $1.84(\mathrm{~m}, 1 \mathrm{H}), 1.47(\mathrm{~s}, 9 \mathrm{H}), 1.43(\mathrm{~s}, 9 \mathrm{H}) ;{ }^{13} \mathrm{C} \mathrm{NMR}\left(100 \mathrm{MHz}, \mathrm{CD}_{2} \mathrm{Cl}_{2}\right): 192.8$ (C), 172.7 (C), 171.8 (C), 171.7 (C), 144.1 (C), 144.0 (C), 138.2 (C), 136.4 (C), 133.4 (C), $131.7(\mathrm{C}), 129.1(\mathrm{C}), 112.7(\mathrm{C}), 82.6(\mathrm{C}), 80.9(\mathrm{C}), 52.9(\mathrm{CH}), 36.8\left(\mathrm{CH}_{2}\right), 31.9\left(\mathrm{CH}_{2}\right), 30.6$ $\left(\mathrm{CH}_{2}\right), 28.4\left(3 \times \mathrm{CH}_{3}\right), 28.3\left(\mathrm{CH}_{2}\right), 28.3\left(3 \times \mathrm{CH}_{3}\right), 16.4\left(\mathrm{CH}_{3}\right), 14.5\left(\mathrm{CH}_{3}\right)$; MS (ESI+, $\mathrm{MeOH}, n / n$ : isotopic peaks): 645/647 $\left(100,[\mathrm{M}+\mathrm{H}]^{+}\right), 589 / 591\left(28,[\mathrm{M}-t \mathrm{Bu}+\mathrm{H}]^{+}\right), 533 / 535$ (11, [M-2tBu+H] $]^{+}$); HRMS (ESI, +ve) calcd for $\mathrm{C}_{27} \mathrm{H}_{34} \mathrm{BrO}_{6} \mathrm{NS}_{3}: 644.0804$, found: 644.0804 .

Compound 26. To a solution of 25 (152 mg, $0.236 \mathrm{mmol})$ in DCM (25 mL), mCPBA (124 mg, $0.718 \mathrm{mmol}$ ) was added in several portions and the mixture was stirred overnight at rt. The reaction was diluted with DCM $(30 \mathrm{~mL})$ and extracted with a saturated solution of $\mathrm{NaHCO}_{3}(3 \times 30 \mathrm{~mL})$. The aqueous layer was extracted with DCM (3 x $\left.30 \mathrm{~mL}\right)$. Combined organic phase was washed with $\mathrm{H}_{2} \mathrm{O}(20 \mathrm{~mL})$ and brine $(20 \mathrm{~mL})$ and finally dried over $\mathrm{Na}_{2} \mathrm{SO}_{4}$. The crude was purified by flash chromatography $\left(\mathrm{SiO}_{2}, \mathrm{PE} /\right.$ acetone 10:0.25) to afford compound 26 as a yellow solid (116 mg, 73\%). $R_{\mathrm{f}}(\mathrm{PE} /$ acetone 10:0.25): 0.1; Mp: 129 $130{ }^{\circ} \mathrm{C}$; IR (neat): 2979 (w), 2927 (w), 2861 (w), 1729 (m), 1654 (m), $15335(\mathrm{w}), 1523(\mathrm{w})$, $1451(\mathrm{w}), 1413(\mathrm{w}), 1392(\mathrm{w}), 1368(\mathrm{~m}), 1310(\mathrm{~m}), 1250(\mathrm{~m}), 1146(\mathrm{~s}), 1102(\mathrm{~m}), 845(\mathrm{w}) ;{ }^{1} \mathrm{H}$ NMR (400 MHz, $\left.\mathrm{CD}_{2} \mathrm{Cl}_{2}\right): 6.30\left(\mathrm{~d},{ }^{3} J(\mathrm{H}, \mathrm{H})=7.9 \mathrm{~Hz}, 1 \mathrm{H}\right), 4.41\left(\mathrm{td},{ }^{3} J(\mathrm{H}, \mathrm{H})=7.9,5.0 \mathrm{~Hz}\right.$, $1 \mathrm{H}), 3.15-3.11(\mathrm{~m}, 2 \mathrm{H}), 2.67(\mathrm{~s}, 3 \mathrm{H}), 2.65-2.62(\mathrm{~m}, 2 \mathrm{H}), 2.34(\mathrm{~s}, 3 \mathrm{H}), 2.31-2.24(\mathrm{~m}, 2 \mathrm{H})$, $2.12-2.03(\mathrm{~m}, 1 \mathrm{H}), 1.91-1.83(\mathrm{~m}, 1 \mathrm{H}), 1.46(\mathrm{~s}, 9 \mathrm{H}), 1.43(\mathrm{~s}, 9 \mathrm{H}) ;{ }^{13} \mathrm{C}$ NMR $(101 \mathrm{MHz}$, $\mathrm{CD}_{2} \mathrm{Cl}_{2}$ ): 192.1 (C), 172.7 (C), 171.6 (C), 171.4 (C), 143.5 (C), 143.5 (C), 141.1 (C), 138.5 (C), $138.4(\mathrm{C}), 133.5(\mathrm{C}), 133.4(\mathrm{C}), 117.2(\mathrm{C}), 82.6(\mathrm{C}), 81.0(\mathrm{C}), 53.0(\mathrm{CH}), 36.9\left(\mathrm{CH}_{2}\right)$, 
$31.9\left(\mathrm{CH}_{2}\right), 30.3\left(\mathrm{CH}_{2}\right), 28.4\left(3 \times \mathrm{CH}_{3}\right), 28.3\left(\mathrm{CH}_{2}\right), 28.2\left(3 \times \mathrm{CH}_{3}\right), 14.5\left(\mathrm{CH}_{3}\right), 12.9\left(\mathrm{CH}_{3}\right)$; MS (ESI+, MeOH, $n / n$ : isotopic peaks): 677/679 $\left(100,[\mathrm{M}+\mathrm{H}]^{+}\right), 621 / 623\left(28,[\mathrm{M}-t \mathrm{Bu}+\mathrm{H}]^{+}\right)$, 565/567 (11, [M-2tBu+H] $]^{+}$); HRMS (ESI, +ve) calcd for $\mathrm{C}_{27} \mathrm{H}_{34} \mathrm{BrO}_{8} \mathrm{~S}_{3}$ : 676.0703, found: 676.0705 .

General Procedure for Stille Coupling (2-8): In a two neck round bottom flask equipped with a stirrer, non-oxidized derivative $(\mathbf{9}, \mathbf{1 1}, \mathbf{1 5}$ or $\mathbf{2 2}, 0.063 \mathrm{M}, 1$ eq) was added and dissolved in dry THF under Ar. The obtained solution was cooled to $-78{ }^{\circ} \mathrm{C}$ and LDA (2 $\mathrm{M}$ in THF, $1.4 \mathrm{eq}$ ) was added. The obtained bright green solution was stirred at $-78{ }^{\circ} \mathrm{C}$ for 60 min, then tributyltin chloride (1.7 eq) was added to obtain a yellow solution which was stirred at $\mathrm{rt}$ for $2 \mathrm{~h}$ under Ar. The solution was then carefully transferred via cannula under Ar in a two neck round bottom flask containing a solution of oxidized derivative $(\mathbf{1 3}, 19$ or $\mathbf{2 6}, 0.076$ $\mathrm{M}, 1.2 \mathrm{eq})$ in freshly distilled $\mathrm{DMF} . \mathrm{Pd}\left(\mathrm{PPh}_{3}\right)_{4}(0.5 \mathrm{eq})$ was added as a solid and the resulting solution was stirred at $70{ }^{\circ} \mathrm{C}$ for 2 days. The mixture was cooled to rt, diluted with $\mathrm{H}_{2} \mathrm{O}$ and extracted with DCM $(3 \mathrm{x})$. The combined organic fractions were washed with $\mathrm{LiCl}$ solution $(5 \%, 4 \mathrm{x})$, dried over $\mathrm{Na}_{2} \mathrm{SO}_{4}$, filtered and concentrated under vacuum to obtain a residue which was purified by flash chromatography $\left(\mathrm{SiO}_{2}+10 \% \mathrm{KF}\right)$ to afford a desired product (28).

Compound 2. Flash chromatography $\left(\mathrm{SiO}_{2}+10 \% \mathrm{KF}\right.$, toluene) to afford compound 2 as an orange solid (38\%). $R_{\mathrm{f}}$ (toluene): 0.15; Mp: decomposes $>230{ }^{\circ} \mathrm{C}$; IR (neat): $2921(\mathrm{w})$, $3862(\mathrm{w}), 1498(\mathrm{w}), 1437(\mathrm{w}), 1403(\mathrm{w}), 1380(\mathrm{~m}), 1310(\mathrm{~s}), 1237(\mathrm{w}), 1160(\mathrm{~m}), 1136(\mathrm{~s})$, 1111 (m), 1077 (w), 1046 (w), 983 (w), 789 (w), 720 (m), 655 (m); ${ }^{1} \mathrm{H}$ NMR (500 MHz, $\left.\mathrm{CD}_{2} \mathrm{Cl}_{2}\right): 3.26\left(\mathrm{q},{ }^{3} J(\mathrm{H}, \mathrm{H})=7.4 \mathrm{~Hz}, 2 \mathrm{H}\right), 2.83\left(\mathrm{q},{ }^{3} J(\mathrm{H}, \mathrm{H})=7.3 \mathrm{~Hz}, 2 \mathrm{H}\right), 2.65(\mathrm{~s}, 3 \mathrm{H}), 2.45$ (s, 3H), $2.39(\mathrm{~s}, 3 \mathrm{H}), 2.36(\mathrm{~s}, 3 \mathrm{H}), 1.38\left(\mathrm{t},{ }^{3} J(\mathrm{H}, \mathrm{H})=7.4 \mathrm{~Hz}, 3 \mathrm{H}\right), 1.27\left(\mathrm{t},{ }^{3} J(\mathrm{H}, \mathrm{H})=7.3 \mathrm{~Hz}\right.$, $3 \mathrm{H}) ;{ }^{13} \mathrm{C}$ NMR (126 MHz, $\mathrm{CD}_{2} \mathrm{Cl}_{2}$ ): 144.7 (C), 143.2 (C), 143.0 (C), 142.6 (C), 140.5 (C), 
138.9 (C), 138.3 (C), 137.2 (C), 137.1 (C), 133.2 (C), 132.8 (C), 132.6 (C), 131.4 (C), 131.1 (C), $131.0(\mathrm{C}), 126.8(\mathrm{C}), 52.4\left(\mathrm{CH}_{2}\right), 33.5\left(\mathrm{CH}_{2}\right), 15.2\left(\mathrm{CH}_{3}\right), 14.4\left(\mathrm{CH}_{3}\right), 14.1\left(\mathrm{CH}_{3}\right), 12.7$ $\left(\mathrm{CH}_{3}\right), 12.6\left(\mathrm{CH}_{3}\right), 8.0\left(\mathrm{CH}_{3}\right)$; MS (ESI+, DCM): $539\left(100,\left[\mathrm{M}-\mathrm{SO}_{2} \mathrm{C}_{2} \mathrm{H}_{6}\right]^{+}\right), 632(100$, $[\mathrm{M}+\mathrm{H}]^{+}$); HRMS (ESI, +ve) calcd for $\mathrm{C}_{24} \mathrm{H}_{22} \mathrm{O}_{4} \mathrm{~S}_{8}^{+}:$629.9278, found: 629.9278 .

Compound 3. Flash chromatography $\left(\mathrm{SiO}_{2}+10 \% \mathrm{KF}\right.$, toluene/EtOAc 10:0.5) to afford compound 3 as a red solid (51\%). $R_{\mathrm{f}}$ (toluene/EtOAc 10:0.5): 0.22; IR (neat): 2924 (w), 1556 (m), 1455 (w), 1434 (w), 1380 (m), 1304 (s), 1247 (m), 1158 (s), 1134 (s), 1069 (m), 1050 (m), 976 (m), 792 (m), 724 (s), 648 (s); ${ }^{1} \mathrm{H}$ NMR (500 MHz, $\mathrm{CD}_{2} \mathrm{Cl}_{2}$ ): 3.98 (s, 3H), 3.26 (q, ${ }^{3} J$ $(\mathrm{H}, \mathrm{H})=7.4 \mathrm{~Hz}, 2 \mathrm{H}), 2.65(\mathrm{~s}, 3 \mathrm{H}), 2.39(\mathrm{~s}, 3 \mathrm{H}), 2.35(\mathrm{~s}, 3 \mathrm{H}), 2.22(\mathrm{~s}, 3 \mathrm{H}), 1.38\left(\mathrm{t},{ }^{3} J(\mathrm{H}, \mathrm{H})=\right.$ 7.4 Hz, 3H); ${ }^{13} \mathrm{C}$ NMR (126 MHz, $\mathrm{CD}_{2} \mathrm{Cl}_{2}$ ): 160.8 (C), 144.8 (C), 143.2 (C), 141.1 (C), 140.7 (C), 140.1 (C), 138.9 (C), 137.2 (C), 132.9 (C), 132.6 (C), 132.5 (C), 132.4 (C), 124.5 (C), $117.7(\mathrm{C}), 113.0(\mathrm{C}), 63.2\left(\mathrm{CH}_{3}\right), 52.5\left(\mathrm{CH}_{2}\right), 14.5\left(\mathrm{CH}_{3}\right), 12.7\left(\mathrm{CH}_{3}\right), 12.6\left(\mathrm{CH}_{3}\right), 10.9\left(\mathrm{CH}_{3}\right)$, $8.0\left(\mathrm{CH}_{3}\right) ; \mathrm{MS}(\mathrm{ESI}+, \mathrm{MeOH}): 601\left(100,[\mathrm{M}+\mathrm{H}]^{+}\right), 509\left(100,\left[\mathrm{C}_{21} \mathrm{H}_{15} \mathrm{O}_{3} \mathrm{~S}_{6}+\mathrm{H}\right]^{+}\right) ; \mathrm{HRMS}(\mathrm{ESI}$, +ve) calcd for $\mathrm{C}_{23} \mathrm{H}_{20} \mathrm{O}_{5} \mathrm{~S}_{7}$ : 599.9350, found: 599.9353 .

Compound 4. Flash chromatography $\left(\mathrm{SiO}_{2}+10 \% \mathrm{KF}\right.$, toluene) to afford compound 4 as a red solid (32\%). $R_{\mathrm{f}}$ (toluene): 0.45; Mp: decomposes $>250{ }^{\circ} \mathrm{C}$; IR (neat): 2962 (w), 2922 (w), 2852 (w), $2214(\mathrm{~m}), 1506(\mathrm{w}), 14401(\mathrm{w}), 1441(\mathrm{~m}), 1415$ (m), $1379(\mathrm{w}), 1318$ (s), 1145 (s), $1088(\mathrm{w}), 655$ (w); ${ }^{1} \mathrm{H}$ NMR (500 MHz, $\left.\mathrm{CD}_{2} \mathrm{Cl}_{2}\right): 2.83\left(\mathrm{q},{ }^{3} J(\mathrm{H}, \mathrm{H})=7.3 \mathrm{~Hz}, 2 \mathrm{H}\right), 2.58(\mathrm{~s}$, 3H), $2.45(\mathrm{~s}, 3 \mathrm{H}), 2.40(\mathrm{~s}, 3 \mathrm{H}), 2.36(\mathrm{~s}, 3 \mathrm{H}), 1.27\left(\mathrm{t},{ }^{3} J(\mathrm{H}, \mathrm{H})=7.3 \mathrm{~Hz}, 3 \mathrm{H}\right) ;{ }^{13} \mathrm{C}$ NMR $(126$ $\mathrm{MHz}, \mathrm{CD}_{2} \mathrm{Cl}_{2}$ ): 145.7 (C), 144.0 (C), 143.1 (C), 142.7 (C), 141.7 (C), 140.8 (C), 138.4 (C), 137.1 (C), 133.3 (C), 133.0 (C), 132.7 (C), 131.5 (C), 131.2 (C), 131.1 (C), 126.7 (C), 113.0 (C), $109.7(\mathrm{C}), 33.6\left(\mathrm{CH}_{2}\right), 15.2\left(\mathrm{CH}_{3}\right), 14.4\left(\mathrm{CH}_{3}\right), 14.1\left(\mathrm{CH}_{3}\right), 13.5\left(\mathrm{CH}_{3}\right), 12.7\left(\mathrm{CH}_{3}\right)$; $\mathrm{MS}$ $(\mathrm{ESI}+, \mathrm{DCM}): 564\left(100,[\mathrm{M}+\mathrm{H}]^{+}\right)$; HRMS (ESI, +ve) calcd for $\mathrm{C}_{23} \mathrm{H}_{17} \mathrm{NO}_{2} \mathrm{~S}_{7}^{+}: 562.9299$ found: 562.9299 . 
Compound 5. Flash chromatography $\left(\mathrm{SiO}_{2}+10 \% \mathrm{KF}\right.$, toluene/DCM 1:0.25) and final wash with acetone to afford compound 5 as a red solid (44\%). $R_{\mathrm{f}}$ (toluene/EtOAc 1:0.25): 0.42; IR (neat): 3736 (w), 2926 (w), 2212 (w), 1556 (m), 1456 (w), 1425 (w), 1379 (m), 1312 (s), 1250 (m), 1143 (s), 1058 (w), 1003 (w); ${ }^{1} \mathrm{H}$ NMR (500 MHz, $\mathrm{CD}_{2} \mathrm{Cl}_{2}$ ): 3.98 (s, 3H), 2.58 (s, 3H), $2.40(\mathrm{~s}, 3 \mathrm{H}), 2.35(\mathrm{~s}, 3 \mathrm{H}), 2.22(\mathrm{~s}, 3 \mathrm{H}) ;{ }^{13} \mathrm{C} \mathrm{NMR}\left(126 \mathrm{MHz}, \mathrm{CD}_{2} \mathrm{Cl}_{2}\right): 160.9(\mathrm{C})$, 145.7 (C), 144.0 (C), 141.7 (C), 141.2 (C), 140.9 (C), 140.1 (C), 139.0 (C), 132.9 (C), 132.7 (C), $132.5(\mathrm{C}), 132.5(\mathrm{C}), 124.5(\mathrm{C}), 117.7(\mathrm{C}), 113.1(\mathrm{C}), 109.5(\mathrm{C}), 63.2\left(\mathrm{CH}_{3}\right), 14.5\left(\mathrm{CH}_{3}\right)$, $13.5\left(\mathrm{CH}_{3}\right), 12.7\left(\mathrm{CH}_{3}\right), 10.9\left(\mathrm{CH}_{3}\right)$; MS (ESI+, DCM): $534\left(100,[\mathrm{M}+\mathrm{H}]^{+}\right)$, MALDI-TOF (DCTB): $532\left(100,[\mathrm{M}]^{+}\right)$.

Compound 6. Flash chromatography $\left(\mathrm{SiO}_{2}+10 \% \mathrm{KF}\right.$, toluene) to afford compound 6 as an orange solid (35\%). $R_{\mathrm{f}}$ (toluene): $0.2 ; \mathrm{Mp}$ : decomposes $>200{ }^{\circ} \mathrm{C}$; IR (neat): $3748(\mathrm{~m})$, 2859 (m), 2240 (m), 2163 (m), 1425 (s), 1318 (s), 1146 (s), 1097 (s), 823 (m), 739 (m), 703 (s), $612(\mathrm{~m}), 573(\mathrm{~m}) ;{ }^{1} \mathrm{H}$ NMR $\left(400 \mathrm{MHz}, \mathrm{CD}_{2} \mathrm{Cl}_{2}\right): 7.13\left(\mathrm{q},{ }^{4} J(\mathrm{H}, \mathrm{H})=0.7 \mathrm{~Hz}, 1 \mathrm{H}\right), 2.57(\mathrm{~s}$, $3 \mathrm{H}), 2.44\left(\mathrm{~d},{ }^{3} J(\mathrm{H}, \mathrm{H})=0.7 \mathrm{~Hz}, 3 \mathrm{H}\right), 2.29(\mathrm{~s}, 3 \mathrm{H}), 2.18(\mathrm{~s}, 3 \mathrm{H}) ;{ }^{13} \mathrm{C} \mathrm{NMR}\left(100 \mathrm{MHz}, \mathrm{CD}_{2} \mathrm{Cl}_{2}\right)$ : 147.9 (C), 143.5 (C), 142.8 (C), 142.2 (C), 140.5 (C), 139.2 (C), 138.8 (C), 138.3 (C), 137.0 (C), $136.4(\mathrm{C}), 133.4(\mathrm{C}), 131.6(\mathrm{C}), 127.6(\mathrm{C}), 119.3\left(\mathrm{CH}_{2}\right), 117.3$ (C), 111.9 (C), 18.4 $\left(\mathrm{CH}_{3}\right), 15.3\left(\mathrm{CH}_{3}\right), 13.4\left(\mathrm{CH}_{3}\right), 12.4\left(\mathrm{CH}_{3}\right)$; MALDI-TOF (DCTB): 503 (100, [M] $\left.{ }^{+}\right)$.

Compound 7. Flash chromatography $\left(\mathrm{SiO}_{2}+10 \% \mathrm{KF}\right.$, toluene/PE $\left.1: 1+10 \% \mathrm{KF}\right)$ to afford compound 7 as a red solid (40\%). $R_{\mathrm{f}}$ (toluene/PE 1:1): $0.25 ; \mathrm{Mp}: 180-181{ }^{\circ} \mathrm{C}$; IR (neat): 2923 (s), 2853 (s), 1679 (m), 1319 (m), 1145 (m), 580 (m), 553 (s); ${ }^{1} \mathrm{H}$ NMR (400 $\mathrm{MHz}, \mathrm{CD}_{2} \mathrm{Cl}_{2}$ ): 2.92-2.98 (m, 4H), 2.58. (s, 3H), 2.39 (s, 3H), 2.35 (s, 3H), $2.26(\mathrm{~s}, 3 \mathrm{H}), 1.47-$ $1.50(\mathrm{~m}, 4 \mathrm{H}), 0.91\left(\mathrm{t},{ }^{3} J(\mathrm{H}, \mathrm{H})=7.3 \mathrm{~Hz}, 6 \mathrm{H}\right),{ }^{13} \mathrm{C} \mathrm{NMR}\left(100 \mathrm{MHz}, \mathrm{CD}_{2} \mathrm{Cl}_{2}\right): 154.8(\mathrm{C}), 145.5$ (C), 143.8 (C), 143.8 (C), 141.1 (C), 140.8 (C), 138.9 (C), 132.8 (C), 132.5 (C), 132.4 (C), 131.3 (C), 129.1 (C), 124.7 (C), 124.1 (C), 124.0 (C), 112.9 (C), 109.3 (C), 60.1 (2 x CH 2 , 
$21.6\left(2 \times \mathrm{CH}_{2}\right), 14.3\left(\mathrm{CH}_{3}\right), 13.3\left(\mathrm{CH}_{3}\right), 12.9\left(\mathrm{CH}_{3}\right), 12.5\left(\mathrm{CH}_{3}\right), 11.7\left(2 \times \mathrm{CH}_{3}\right)$; MALDITOF (DCTB): $602\left(100,[\mathrm{M}]^{+}\right)$.

Compound 8. Flash chromatography $\left(\mathrm{SiO}_{2}, \mathrm{PE} /\right.$ acetone 8.2:1.8) to afford compound $\mathbf{8}$ as a red solid (14\%). $R_{\mathrm{f}}\left(\mathrm{PE} /\right.$ acetone 8.2:1.8): 0.21; Mp: $113-114{ }^{\circ} \mathrm{C}$; IR (neat): $3317(\mathrm{w}), 2966$ (w), 2923 (m), 2855 (w), 1730 (m), 1654 (m), 1543 (w), 1534 (w), 1448 (w), 1376 (m), 1312 (m), 1248 (m), 1145 (s), 1096 (m); ${ }^{1} \mathrm{H}$ NMR (400 MHz, DMSO-d $): 8.22\left(\mathrm{~d},{ }^{3} J(\mathrm{H}, \mathrm{H})=7.7\right.$ Hz, 1H), $4.15-410(\mathrm{~m}, 1 \mathrm{H}), 3.14-3.10(\mathrm{~m}, 2 \mathrm{H}), 2.94-2.91(\mathrm{~m}, 4 \mathrm{H}), 2.62(\mathrm{~s}, 3 \mathrm{H}), 2.59-2.51$ (m, 2H), $2.35(\mathrm{~s}, 3 \mathrm{H}), 2.33(\mathrm{~s}, 3 \mathrm{H}), 2.29-2.25(\mathrm{~m}, 2 \mathrm{H}), 2.23(\mathrm{~s}, 3 \mathrm{H}), 1.94-1.86(\mathrm{~m}, 1 \mathrm{H}), 1.78$ - $1.69(\mathrm{~m}, 1 \mathrm{H}), 1.50-1.40(\mathrm{~m}, 4 \mathrm{H}), 1.39(\mathrm{~s}, 9 \mathrm{H}), 1.39(\mathrm{~s}, 9 \mathrm{H}), 0.88\left(\mathrm{t},{ }^{3} J(\mathrm{H}, \mathrm{H})=7.4 \mathrm{~Hz}, 6 \mathrm{H}\right)$; ${ }^{13} \mathrm{C}$ NMR (126 MHz, DMSO-d $\left.{ }_{6}\right): 192.2(\mathrm{C}), 171.4(\mathrm{C}), 171.0(2 \times \mathrm{C}), 153.8(\mathrm{C}), 143.5$ (C), 142.4 (C), 141.8 (C), 140.2 (C), 140.0 (C), 137.9 (C), 137.6 (C), 135.9 (C), 133.3 (C), 131.4 (C), $131.3(\mathrm{C}), 130.6(\mathrm{C}), 124.7(\mathrm{C}), 122.8(\mathrm{C}), 122.7(\mathrm{C}), 80.6(\mathrm{C}), 79.8(\mathrm{C}), 58.9\left(2 \times \mathrm{CH}_{2}\right)$, $51.8(\mathrm{CH}), 35.9\left(\mathrm{CH}_{2}\right), 31.0\left(\mathrm{CH}_{2}\right), 29.6\left(\mathrm{CH}_{2}\right), 27.7\left(3 \times \mathrm{CH}_{3}\right), 27.6\left(3 \times \mathrm{CH}_{3}\right), 26.4\left(\mathrm{CH}_{2}\right)$, $20.7\left(2 \times \mathrm{CH}_{2}\right), 13.9\left(\mathrm{CH}_{3}\right), 13.7\left(\mathrm{CH}_{3}\right), 12.5\left(\mathrm{CH}_{3}\right), 12.0\left(\mathrm{CH}_{3}\right), 11.4\left(2 \times \mathrm{CH}_{3}\right)$; MS (ESI+, MeOH): $920\left(100,[\mathrm{M}+\mathrm{H}]^{+}\right), 864\left(51,[\mathrm{M}-t \mathrm{Bu}+\mathrm{H}]^{+}\right), 808\left(48,[\mathrm{M}-2 t \mathrm{Bu}+\mathrm{H}]^{+}\right) ;$MALDI-TOF (DCTB): $918\left(100,[\mathrm{M}]^{+}\right)$. 


\section{Solvatochromism}

In a typical experiment, aliquots of a solution of probe in $\mathrm{CHCl}_{3}$ were diluted with air saturated solvents of interest. The solutions were stirred at $25 \pm 0.1{ }^{\circ} \mathrm{C}$ for 5 minutes before the spectra (absorption and emission) were acquired. The concentration of the samples was adjusted to have absorbance $<0.1$.
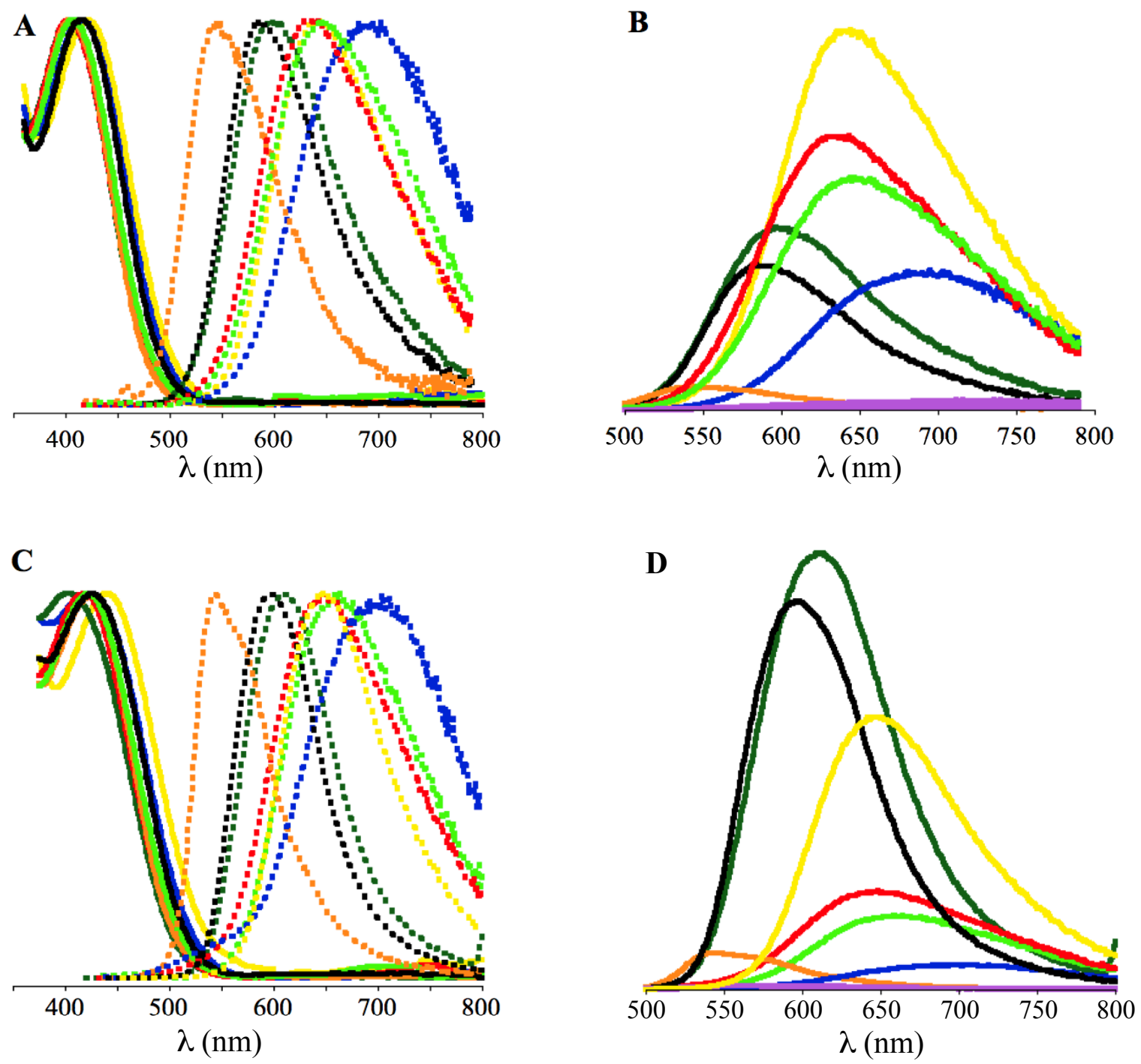

Figure S1. (A and C) Normalized absorption (solid) and emission spectra (dashed) in $\mathrm{CHCl}_{3}$ (yellow), EtOAc (red), toluene (black), THF (light green), DCM (blue), $\mathrm{Et}_{2} \mathrm{O}$ (dark green) and hexane (orange), (B and D) not normalized emission spectra in the same solvent as in (A and B) (same color) and $\mathrm{ACN}$, acetone, $\mathrm{MeOH}, \mathrm{DMF}$ and DMSO in purple of compound 2 (A and $\mathrm{B})$ and $\mathbf{3}(\mathrm{C}$ and $\mathrm{D})$. 

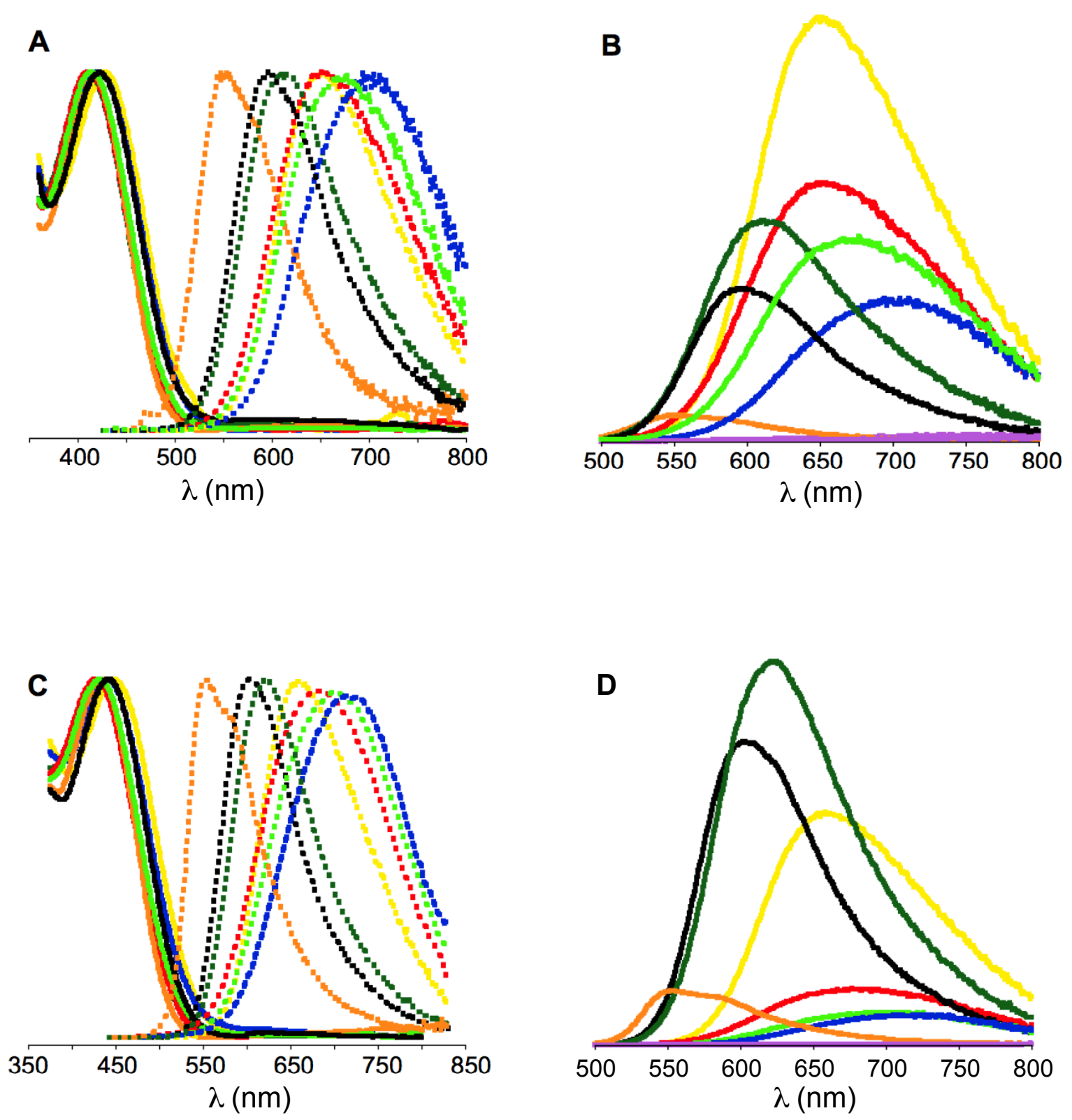

Figure S2. (A and C) Normalized absorption (solid) and emission spectra (dashed) in $\mathrm{CHCl}_{3}$ (yellow), EtOAc (red), toluene (black), THF (light green), DCM (blue), $\mathrm{Et}_{2} \mathrm{O}$ (dark green) and hexane (orange), (B and D) not normalized emission spectra in the same solvent as in (A and B) (same color) and $\mathrm{ACN}$, acetone, $\mathrm{MeOH}, \mathrm{DMF}$ and DMSO in purple of compound 4 (A and $\mathrm{B})$ and $\mathbf{5}(\mathrm{C}$ and $\mathrm{D})$. 
A

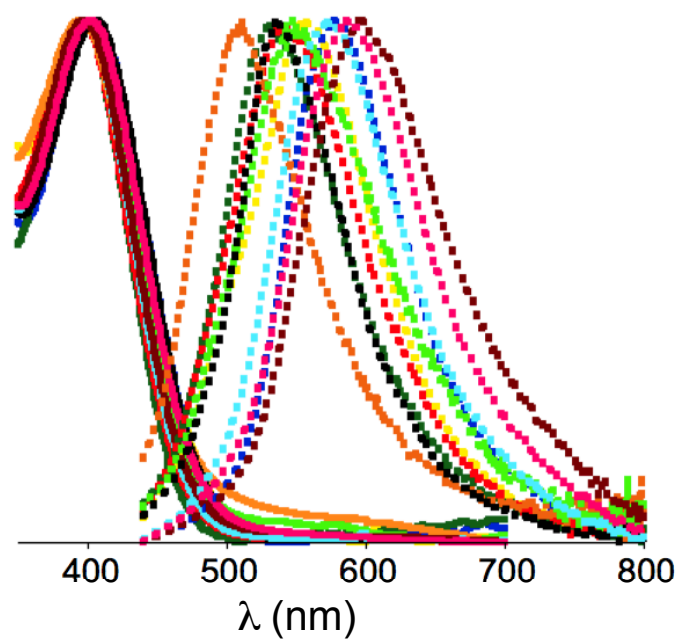

B

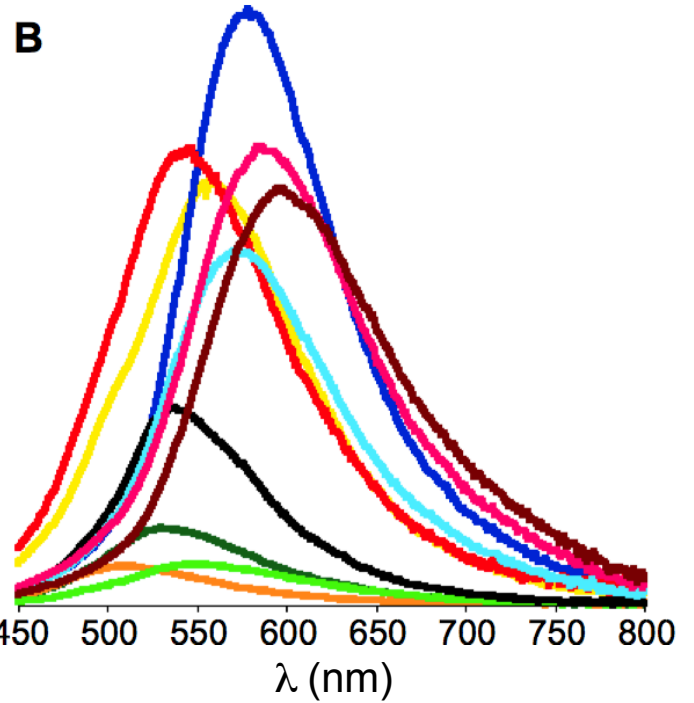

Figure S3. A) Normalized absorption (solid) and emission spectra (dashed) of compound 6 recorded in $\mathrm{CHCl}_{3}$ (yellow), EtOAc (red), toluene (black), THF (light green), DCM (blue), $\mathrm{Et}_{2} \mathrm{O}$ (dark green), hexane (orange), acetone (light blue), $\mathrm{MeOH}$ (carmine) and DMF (pink), B) not normalized emission spectra in the same solvent as in (A) (same color).
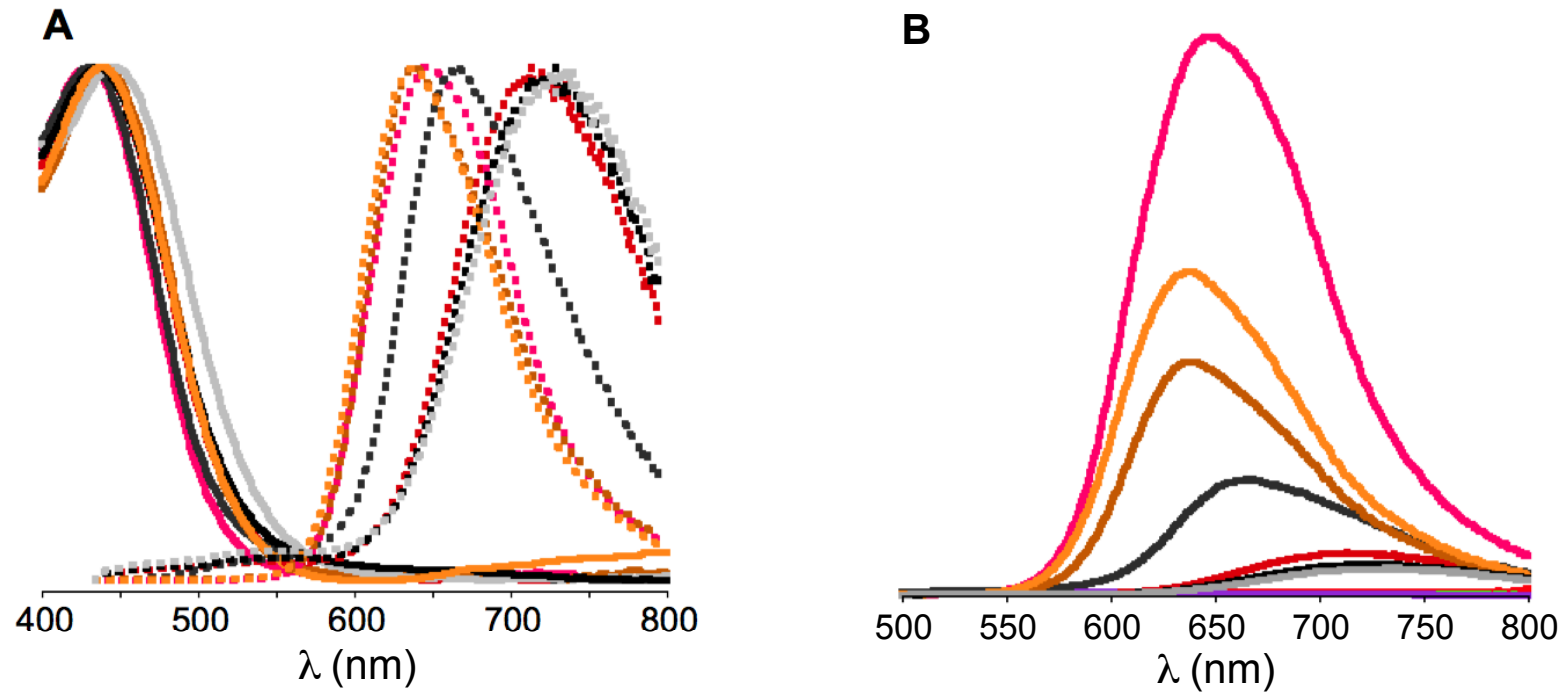

Figure S4. A) Normalized absorption (solid) and emission spectra (dashed) of compound 7 recorded in xylene (dark red), heptane (dark pink), toluene (black), $\mathrm{CCl}_{4}$ (dark grey), cyclohexane (light brown), benzene (light grey), hexane (orange B) not normalized emission spectra in the same solvent as in (A) (same color) and $\mathrm{ACN}$, acetone, $\mathrm{MeOH}, \mathrm{DMF}, \mathrm{CHCl}_{3}$, EtOAc, DCM and $\mathrm{Et}_{2} \mathrm{O}$ in purple. 
A

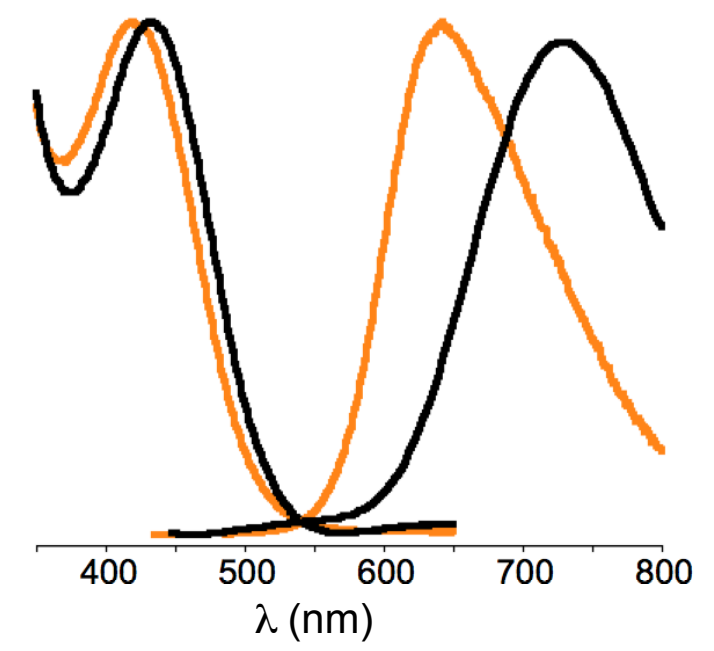

B

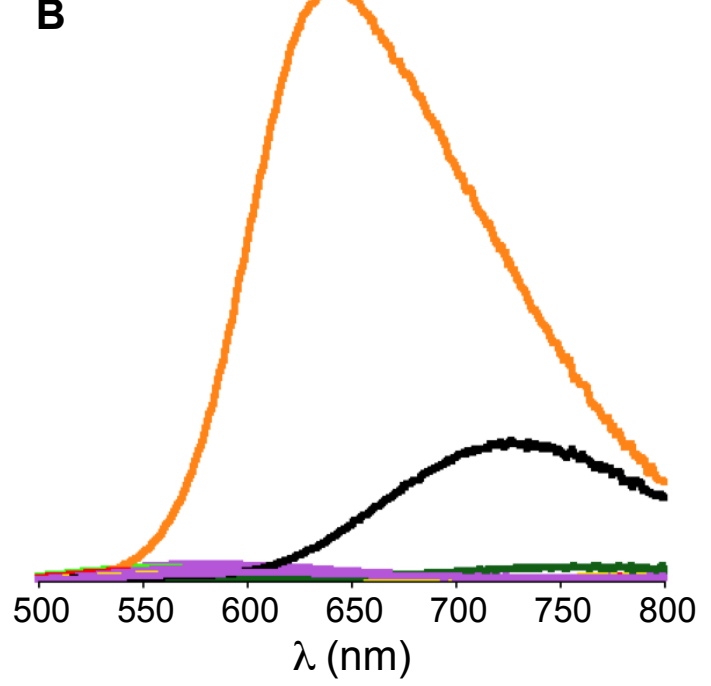

Figure S5. A) Normalized absorption (solid) and emission spectra (dashed) of compound 8 recorded in toluene (black), hexane (orange), B) not normalized emission spectra in the same solvent as in (A) (same color) and $\mathrm{CHCl}_{3}$ (yellow), EtOAc (red), THF (light green), DCM (blue), $\mathrm{Et}_{2} \mathrm{O}$ (dark green), and $\mathrm{ACN}$, acetone, $\mathrm{MeOH}, \mathrm{DMF}$ and DMSO in purple. 
Table S1. Absorption and emission maxima in different solvents.

\begin{tabular}{|c|c|c|c|c|c|c|c|c|}
\hline solvant & Cpd & $\begin{array}{l}\lambda_{\mathrm{abs}} \\
(\mathrm{nm})^{\mathrm{a}}\end{array}$ & $\begin{array}{l}\lambda_{\mathrm{em}} \\
(\mathrm{nm})^{\mathrm{b}}\end{array}$ & $\begin{array}{l}\Delta v \\
\left(\mathrm{~cm}^{-1}\right)^{\mathrm{c}}\end{array}$ & Cpd & $\begin{array}{l}\lambda_{\mathrm{abs}} \\
(\mathrm{nm})^{\mathrm{a}}\end{array}$ & $\begin{array}{l}\lambda_{\mathrm{em}} \\
(\mathrm{nm})^{\mathrm{b}}\end{array}$ & $\begin{array}{l}\Delta v \\
\left(\mathrm{~cm}^{-1}\right)^{\mathrm{c}}\end{array}$ \\
\hline $\mathrm{CHCl}_{3}$ & 2 & 422 & 643 & 8300 & 3 & 440 & 648 & 7500 \\
\hline DCM & & 416 & 690 & 9900 & & 424 & 704 & 9600 \\
\hline hexane & & 404 & 549 & 6500 & & 422 & 545 & 5400 \\
\hline toluene & & 416 & 584 & 7200 & & 426 & 596 & 6900 \\
\hline THF & & 407 & 646 & 9300 & & 421 & 658 & 8900 \\
\hline EtOAc & & 403 & 635 & 9300 & & 418 & 648 & 8700 \\
\hline ether & & 403 & 597 & 8300 & & 419 & 613 & 7700 \\
\hline $\mathrm{CHCl}_{3}$ & 4 & 428 & 650 & 8300 & 5 & 449 & 658 & 7400 \\
\hline DCM & & 421 & 700 & 9800 & & 440 & 717 & 9000 \\
\hline hexane & & 414 & 554 & 6200 & & 433 & 553 & 5200 \\
\hline toluene & & 422 & 596 & 7100 & & 442 & 603 & 6300 \\
\hline THF & & 414 & 674 & 9700 & & 433 & 694 & 9100 \\
\hline EtOAc & & 411 & 650 & 9400 & & 428 & 681 & 9000 \\
\hline ether & & 411 & 611 & 8300 & & 428 & 621 & 7400 \\
\hline $\mathrm{CHCl}_{3}$ & 6 & 403 & 557 & 7100 & & & & \\
\hline DCM & & 406 & 578 & 7500 & & & & \\
\hline hexane & & 395 & 508 & 5700 & & & & \\
\hline toluene & & 406 & 536 & 6000 & & & & \\
\hline THF & & 400 & 548 & 7000 & & & & \\
\hline EtOAc & & 396 & 543 & 7000 & & & & \\
\hline ether & & 396 & 531 & 6800 & & & & \\
\hline xylene & 7 & 437 & 710 & 9100 & 8 & - & - & - \\
\hline cyclohexane & & 431 & 637 & 7700 & & - & - & - \\
\hline hexane & & 445 & 635 & 6900 & & 420 & 642 & 8400 \\
\hline toluene & & 437 & 729 & 9200 & & 432 & 727 & 9600 \\
\hline $\mathrm{CCl}_{4}$ & & 439 & 664 & 7800 & & - & - & - \\
\hline EtOAc & & 421 & 629 & 7900 & & 418 & 554 & 6400 \\
\hline benzene & & 437 & 738 & 9300 & & - & - & - \\
\hline heptane & & 427 & 647 & 8200 & & - & - & - \\
\hline
\end{tabular}

${ }^{\mathrm{a}}$ Wavelength $\lambda_{\mathrm{abs}}$ of absorption maximum. ${ }^{\mathrm{b}}$ Wavelength $\lambda_{\mathrm{em}}$ of emission maximum (excitation at $\left.\lambda_{\mathrm{abs}}\right){ }^{\mathrm{c}}$ Stokes shift in frequency (wavenumbers, $v_{\mathrm{abs}}-v_{\mathrm{em}}$ ). 
The polarity index $f(\varepsilon)$ was calculated for each solvent with the Equation S1. The wavenumber $\left(\mathrm{cm}^{-1}\right)$ of absorption maximum $v_{\mathrm{a}}$ and that of emission maximum $v_{\mathrm{f}}$ of each solvent are plotted against $f(\varepsilon)$ and linearly correlated (Figure S6). The obtained slopes of the dependence of emission maxima to $f(\varepsilon)$ are summarized in Table S2. Corresponding slopes for absorption maxima were $\sim 0$.

$$
f(\varepsilon)=\frac{\varepsilon_{\mathrm{r}}-1}{2 \varepsilon_{\mathrm{r}}+1}
$$

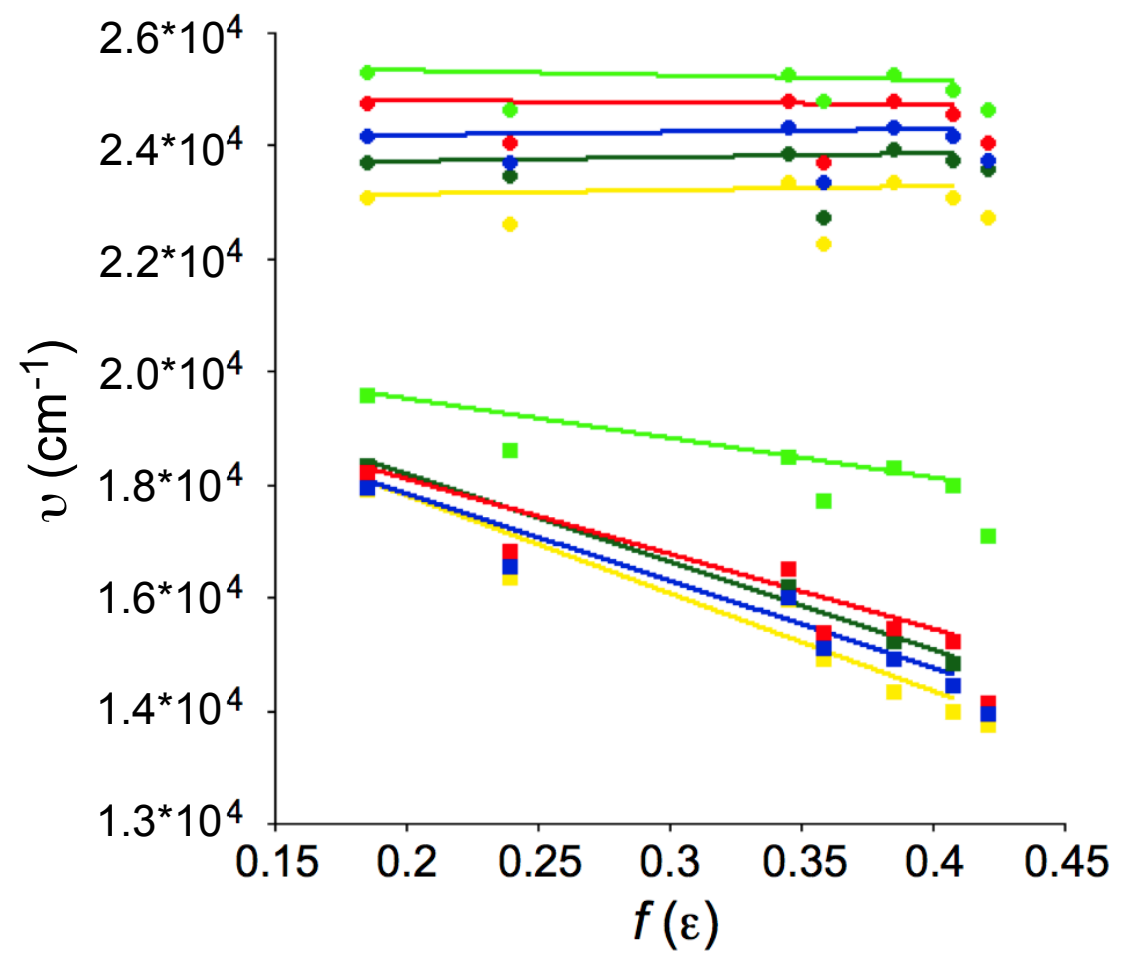

Figure S6. Wavenumber of absorption maximum (circles) and that of emission maximum (square) of $\mathbf{2}$ (red), $\mathbf{3}$ (green), 4 (blue), $\mathbf{5}$ (yellow), $\mathbf{6}$ (light green).

Table S2. Slope of the dependence of emission maxima to the polarity index.

$\begin{array}{cc}\text { cpd } & \text { slope } v_{\mathrm{f}}\left(\mathrm{cm}^{-1}\right) \\ \mathbf{2} & 13300 \\ \mathbf{3} & 15500 \\ \mathbf{4} & 15300 \\ \mathbf{5} & 17400 \\ \mathbf{6} & 6900\end{array}$


Comment: Independence of absorption maxima to the polarity of solvents indicates the absence of push-pull polarization of the chromophore, and thus is consistent with the twisted conformation in the ground state. In contrast, the slopes obtained for the emission spectra correlate well with the expected strength of dipole moment, and are consistent with the polarization of the chromophore upon planarization in the excited state. Fitting of the slopes was done without the solvents with higher refracting index $\left(\mathrm{CHCl}_{3}, \mathrm{DCM}\right.$, toluene).

\section{Femtosecond Time-Resolved Broadband Fluorescence Experiments}

Fluorescence up-conversion with broadband detection was performed with a setup similar to that reported in reference S4 and will be described in detail in future work. In brief, excitation was performed with $100 \mathrm{fs}$ pulses at $400 \mathrm{~nm}$ generated by frequency doubling part of the output of a standard $1 \mathrm{kHz}$ Ti:Sapphire amplified system. The pump intensity on the sample was below $1 \mathrm{~mJ} / \mathrm{cm}^{2}$. The gate pulses were at $1340 \mathrm{~nm}$ and were produced by an optical parametric amplifier (TOPAS C, Light Conversion). Detection of the up-converted spectra was performed with a CCD camera (Andor, DV420A-BU). The full width at half maximum of the cross correlation of the gate with the solvent Raman signal was approximately 170 fs. Corrected time-resolved emission spectra were obtained by calibration with secondary emissive standards as described in reference S4. Time-resolved emission spectra were recorded in two sequential measurements: a linear time-step range from -2 to 2 ps and a logarithmic time-grid covering the remainder of the time-window.

The temporal chirp was determined measuring the instantaneous response of BBOT (Radiant Dyes, used as received) in all of the used solvents. The experimental raw-data were henceforth corrected for the temporal chirp and then transferred into corrected spectra vs. wavelength or wavenumber according to reference S4. The emission maxima were determined by fitting a lognorm-function to the experimental emission spectra vs. 
wavenumber at each time-delay ref. S5.

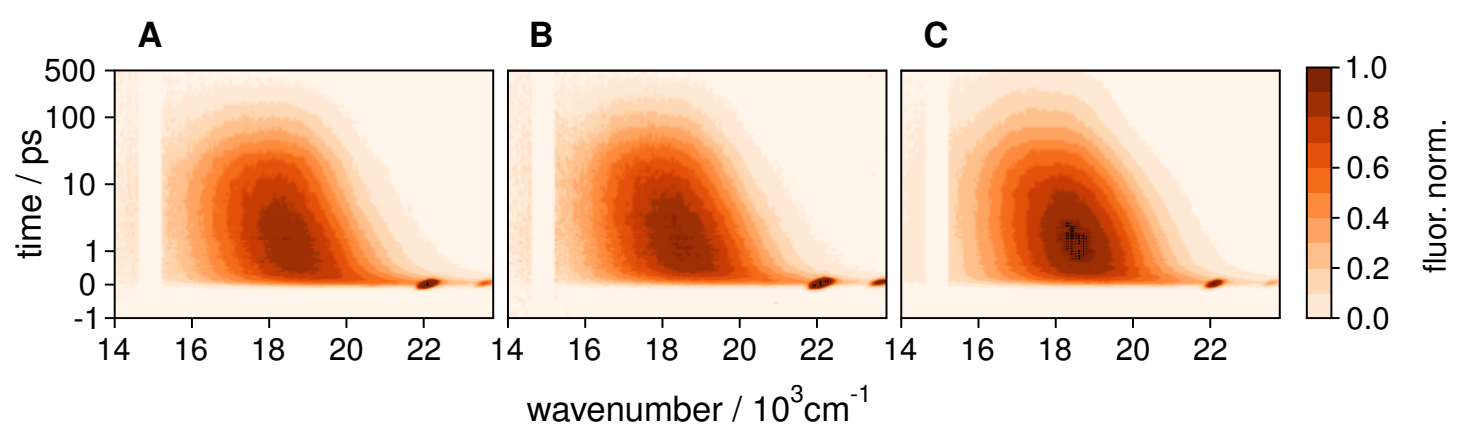

Figure S7. Broadband fluorescence spectra of cpd. 2 in $n$-hexane (A), cyclohexane (B) and $n$ hexadecane (C).

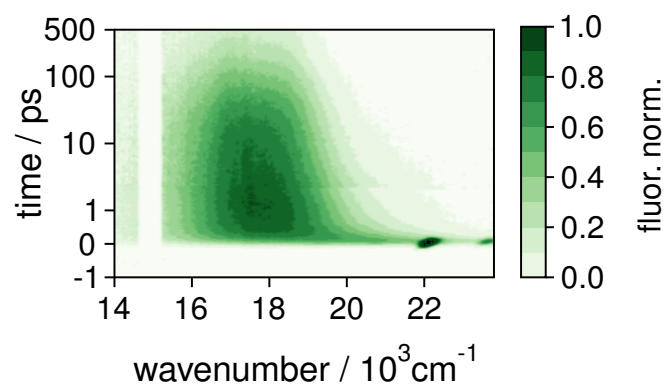

Figure S8. Broadband fluorescence spectrum of cpd. 3 cyclohexane. 


\section{Supporting References}

S1. Dal Molin, M.; Verolet, Q.; Colom, A.; Letrun, R.; Derivery, E.; Gonzalez-Gaitan, M.; Vauthey, E.; Roux, A.; Sakai, N.; Matile, S. J. Am. Chem. Soc. 2015, 137, 568 - 571.

S2. Barbarella, G.; Favaretto, L.; Sotgiu, G. Chem. Mater. 2001, 13, 4112 - 4122.

S3. Wang, H.; Lin, H.; Xu, W.; Zhu, D. Chem. Eur. J. 2013, 19, 3366 - 3373.

S4. Zhang, X.-X.; Würth, C.; Zhao, L.; Resch-Genger, U.; Ernsting, N. P.; Sajadi, M. Rev. Sci. Instr. 2011, 82, 063108.

S5. Horng, M. L.; Gardecki, J. A.; Papazyan, A.; Maroncelli, M. J. Phys. Chem. 1995, 99, $17311-17337$. 
6. NMR Spectra

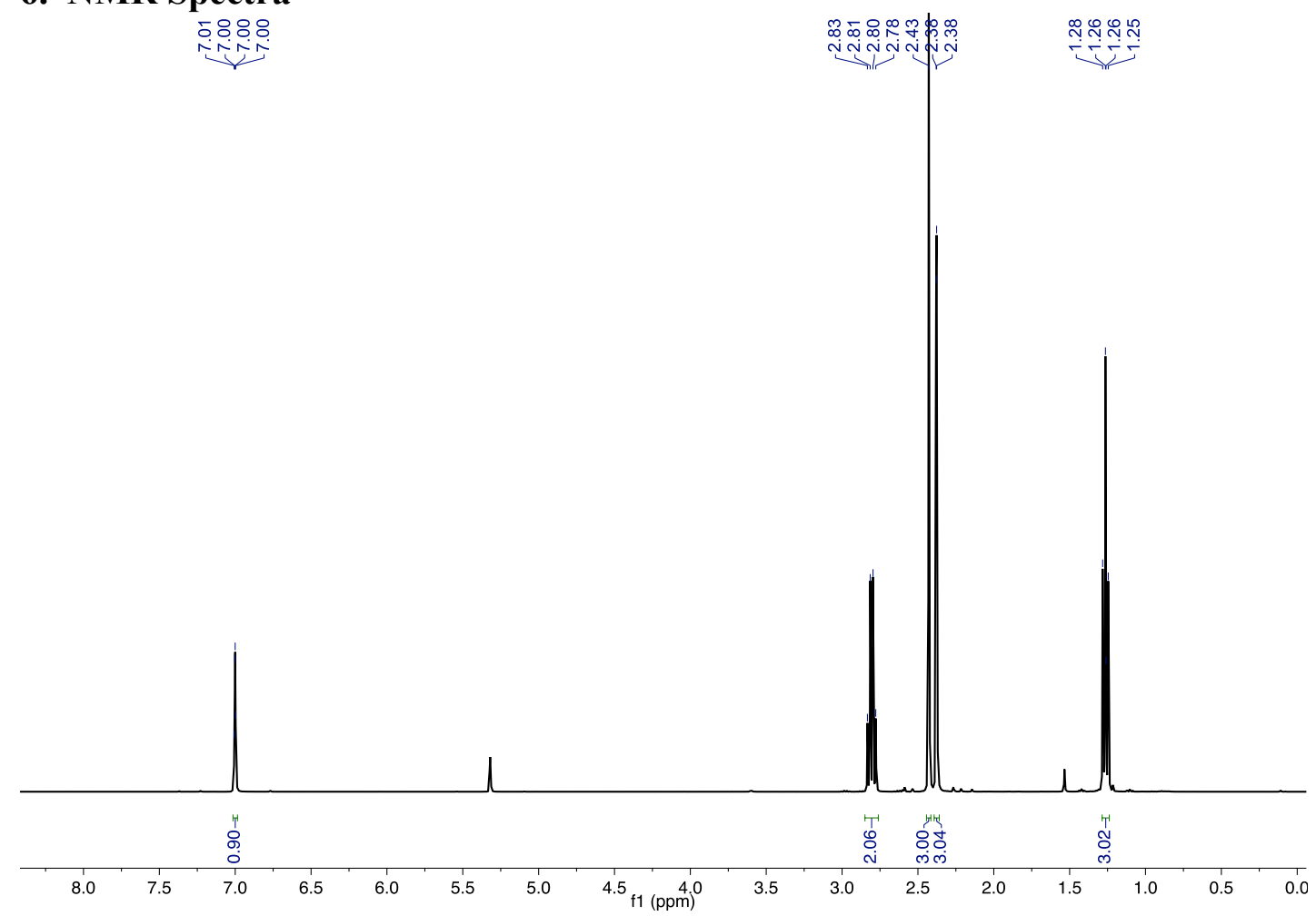

Figure S9. ${ }^{1} \mathrm{H}$ NMR spectrum of 11 in $\mathrm{CD}_{2} \mathrm{Cl}_{2}$.

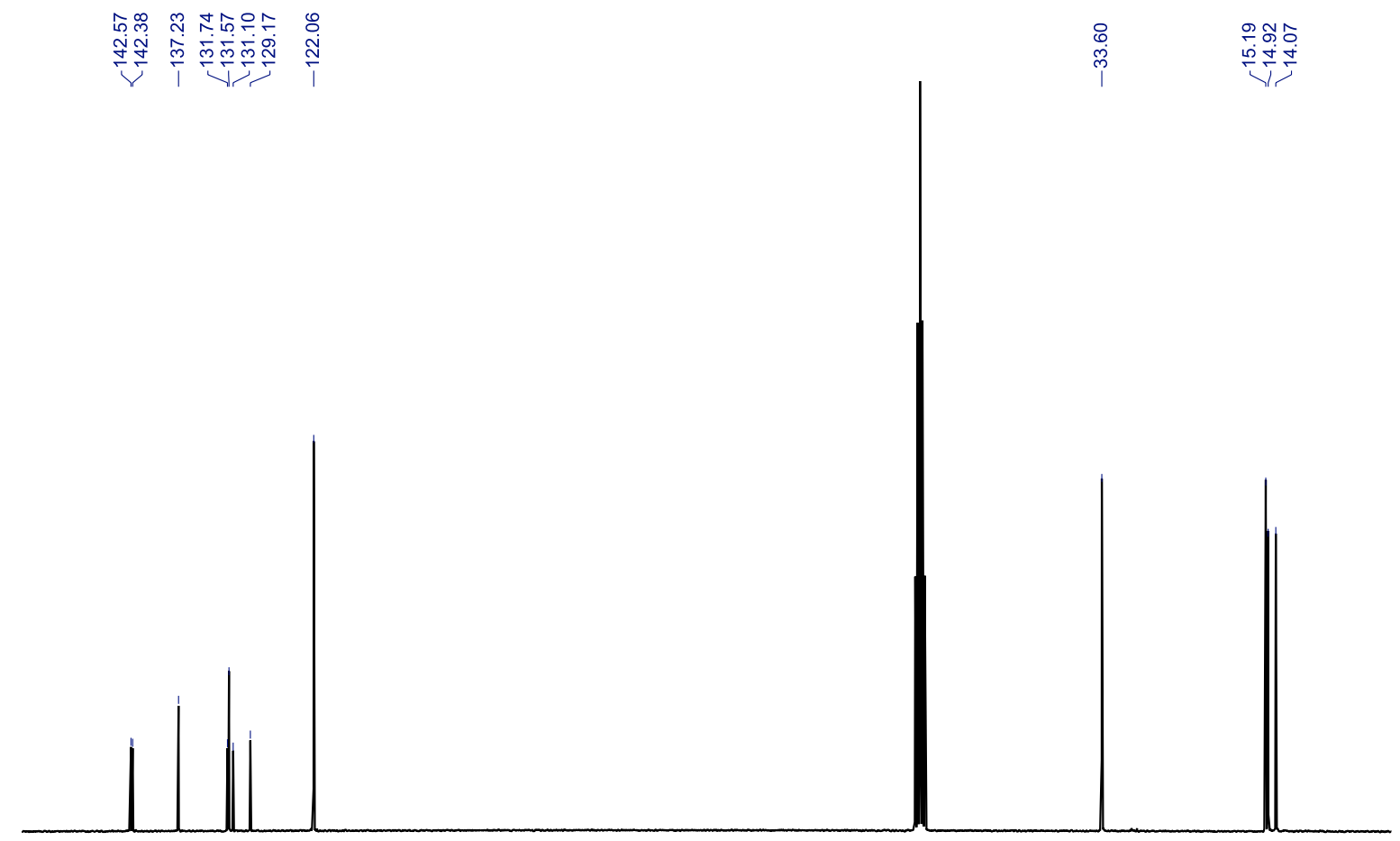

$\begin{array}{llllllllllllllllllllllllllllllllllllllll}150 & 145 & 140 & 135 & 130 & 125 & 120 & 115 & 110 & 105 & 100 & 95 & 90 & 85 & 80 & 75 & 70 & 65 & 60 & 55 & 50 & 45 & 40 & 35 & 30 & 25 & 20 & 15 & 10 & 5\end{array}$

Figure S10. ${ }^{13} \mathrm{C}$ NMR spectrum of 11 in $\mathrm{CD}_{2} \mathrm{Cl}_{2}$. 


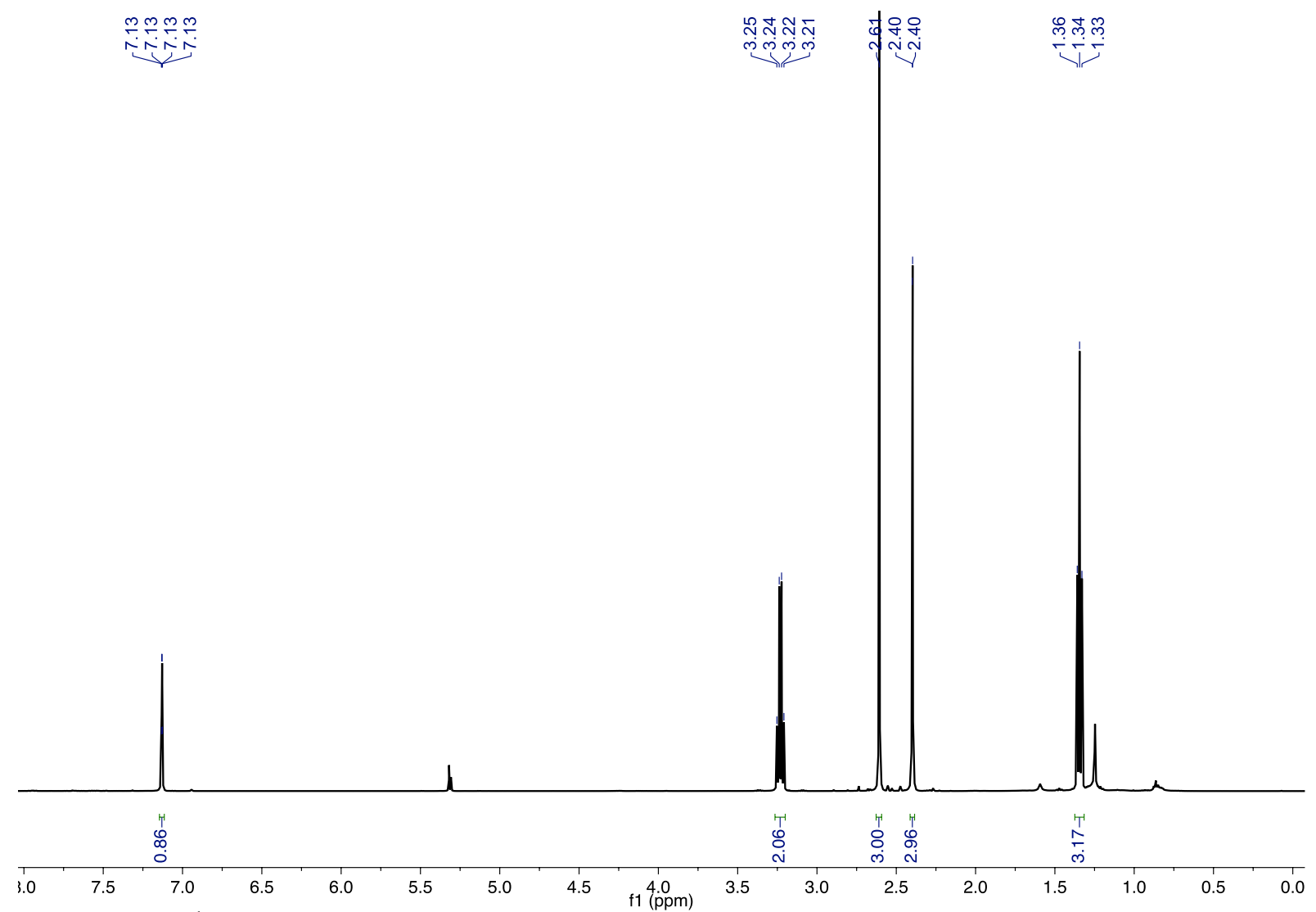

Figure S11. ${ }^{1} \mathrm{H}$ NMR spectrum of 12 in $\mathrm{CD}_{2} \mathrm{Cl}_{2}$.

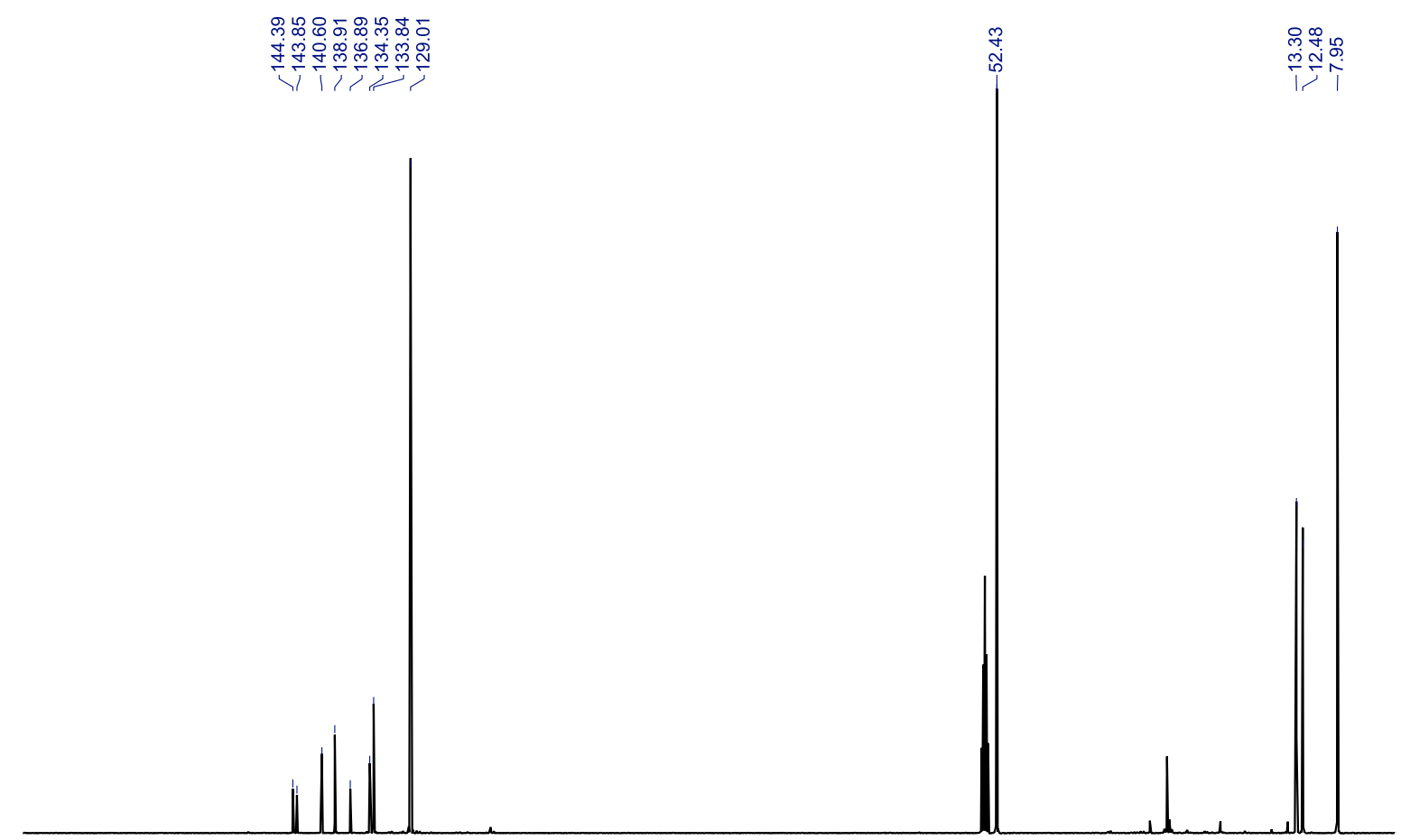

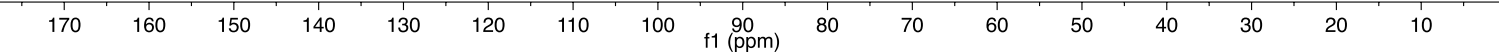

Figure S12. ${ }^{13} \mathrm{C}$ NMR spectrum of 12 in $\mathrm{CD}_{2} \mathrm{Cl}_{2}$. 


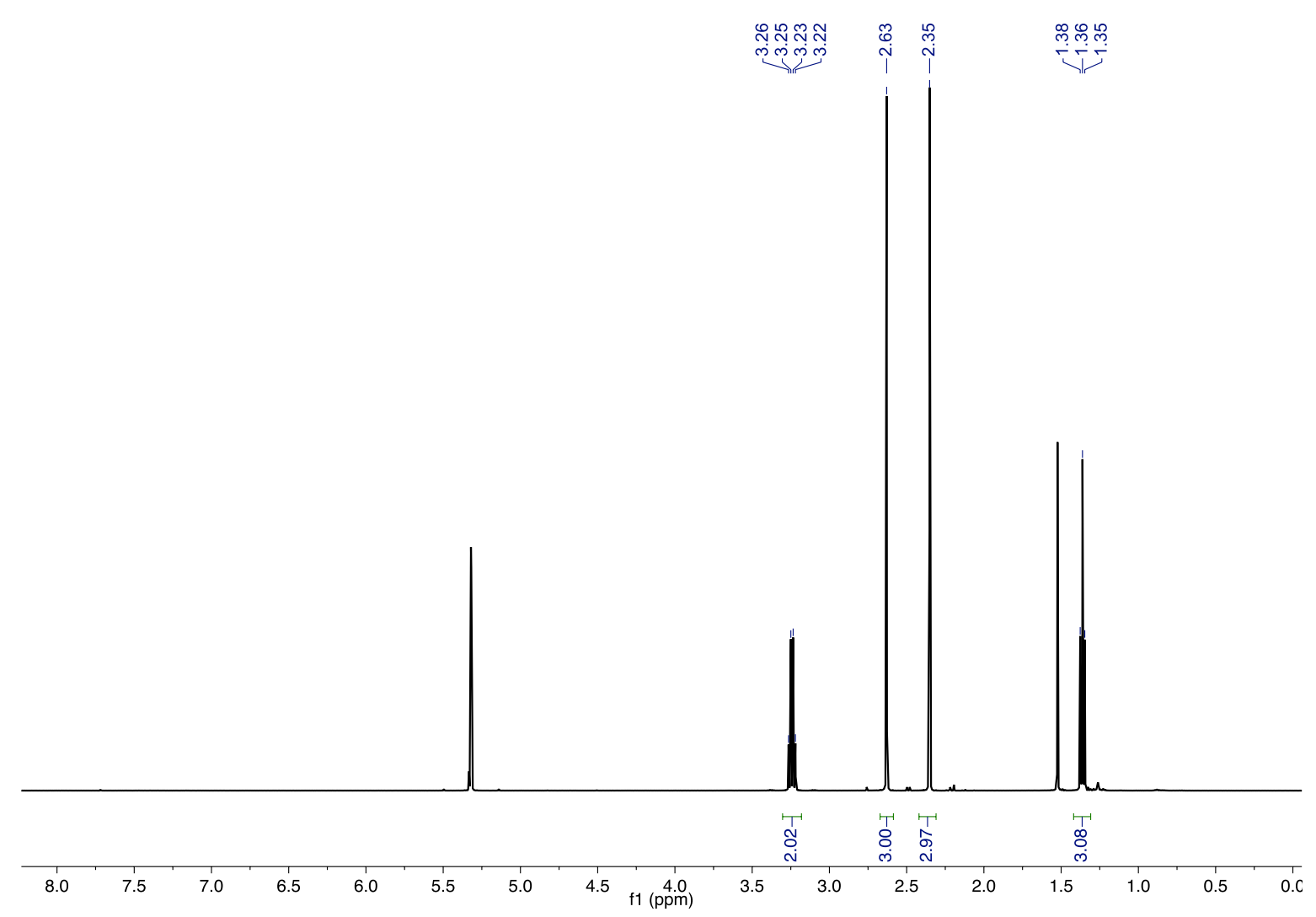

Figure S13. ${ }^{1} \mathrm{H}$ NMR spectrum of 13 in $\mathrm{CD}_{2} \mathrm{Cl}_{2}$.

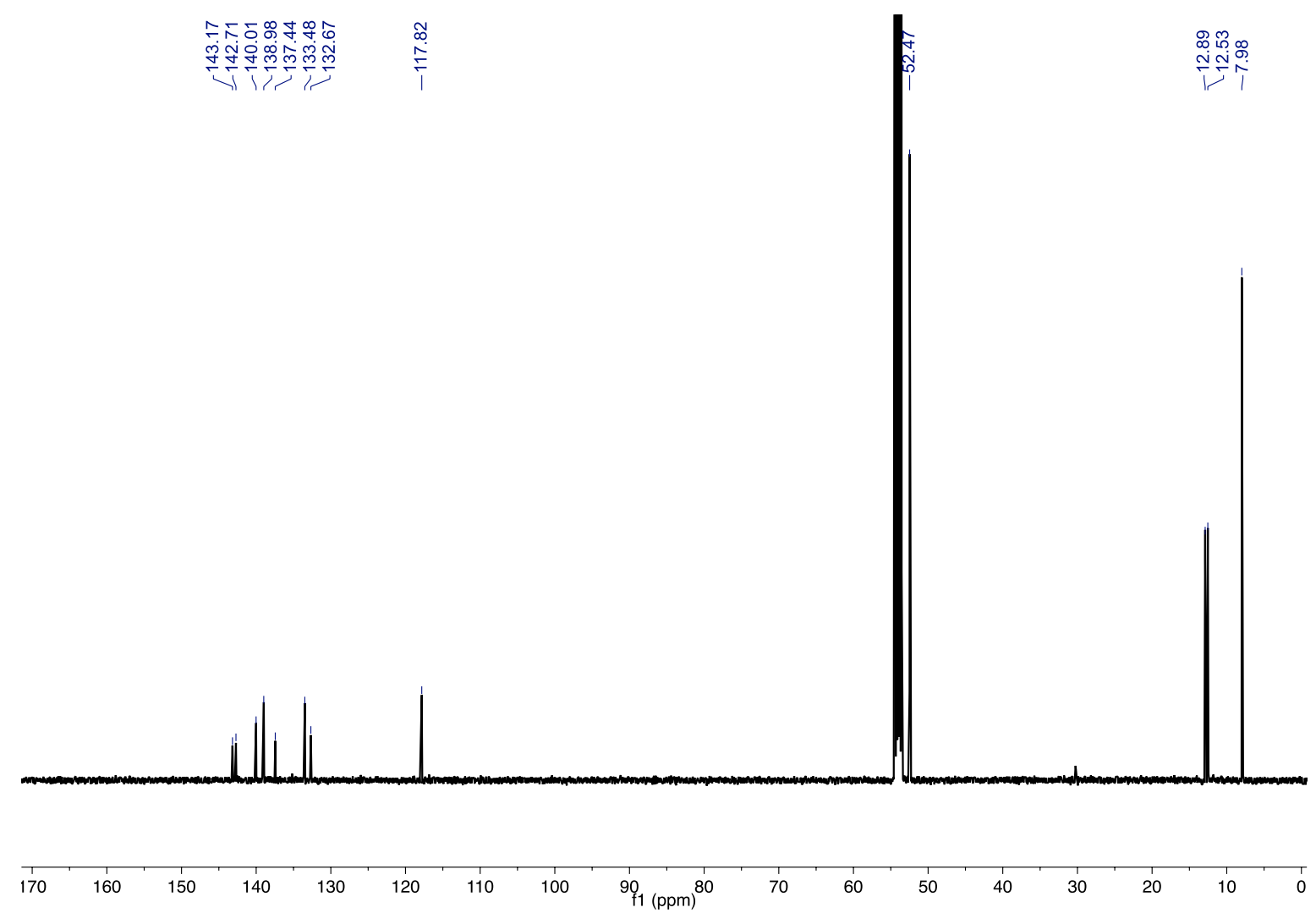

Figure S14. ${ }^{13} \mathrm{C}$ NMR spectrum of 13 in $\mathrm{CD}_{2} \mathrm{Cl}_{2}$. 


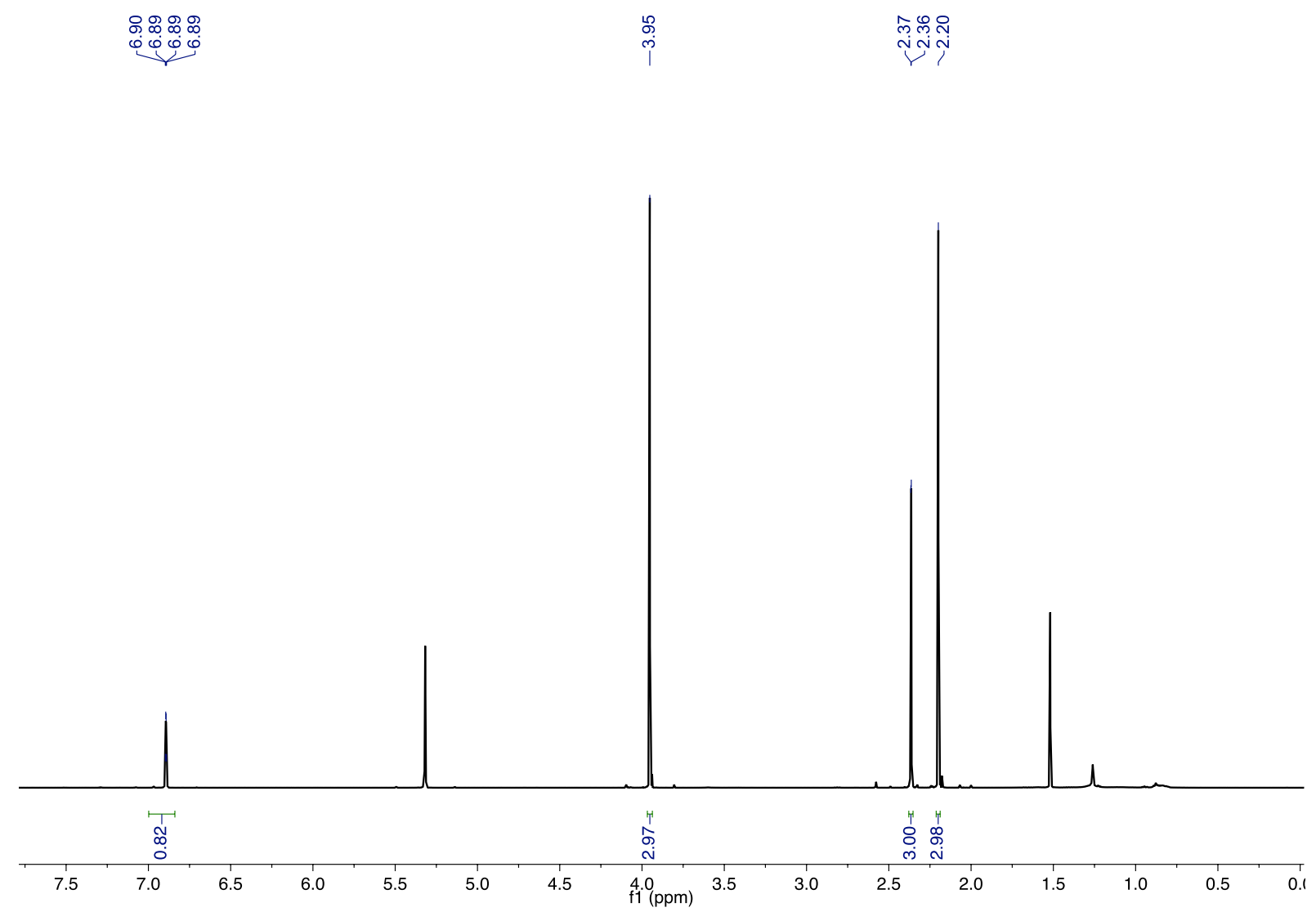

Figure S15. ${ }^{1} \mathrm{H}$ NMR spectrum of 15 in $\mathrm{CD}_{2} \mathrm{Cl}_{2}$.

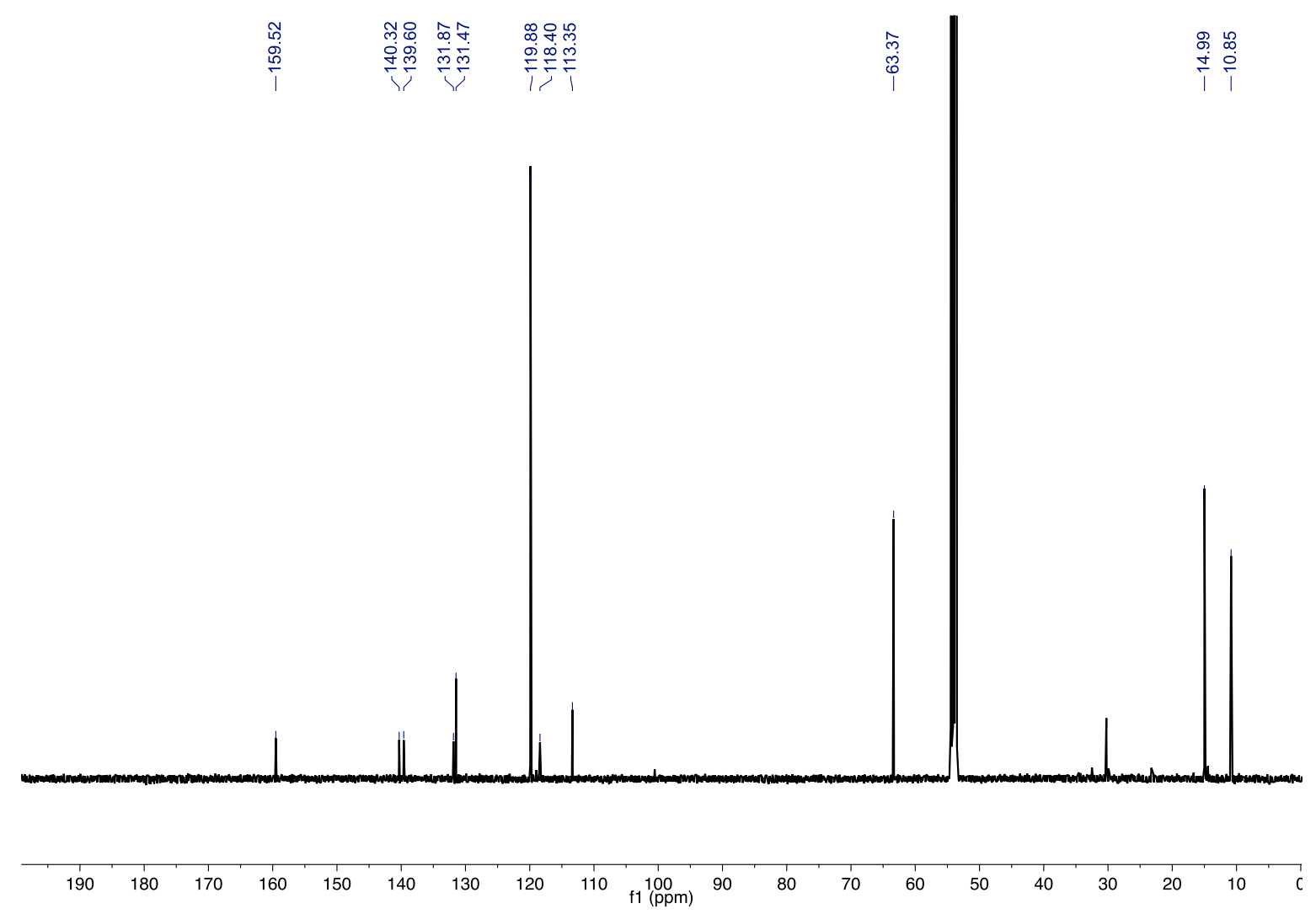

Figure S16. ${ }^{13} \mathrm{C}$ NMR spectrum of 15 in $\mathrm{CD}_{2} \mathrm{Cl}_{2}$. 


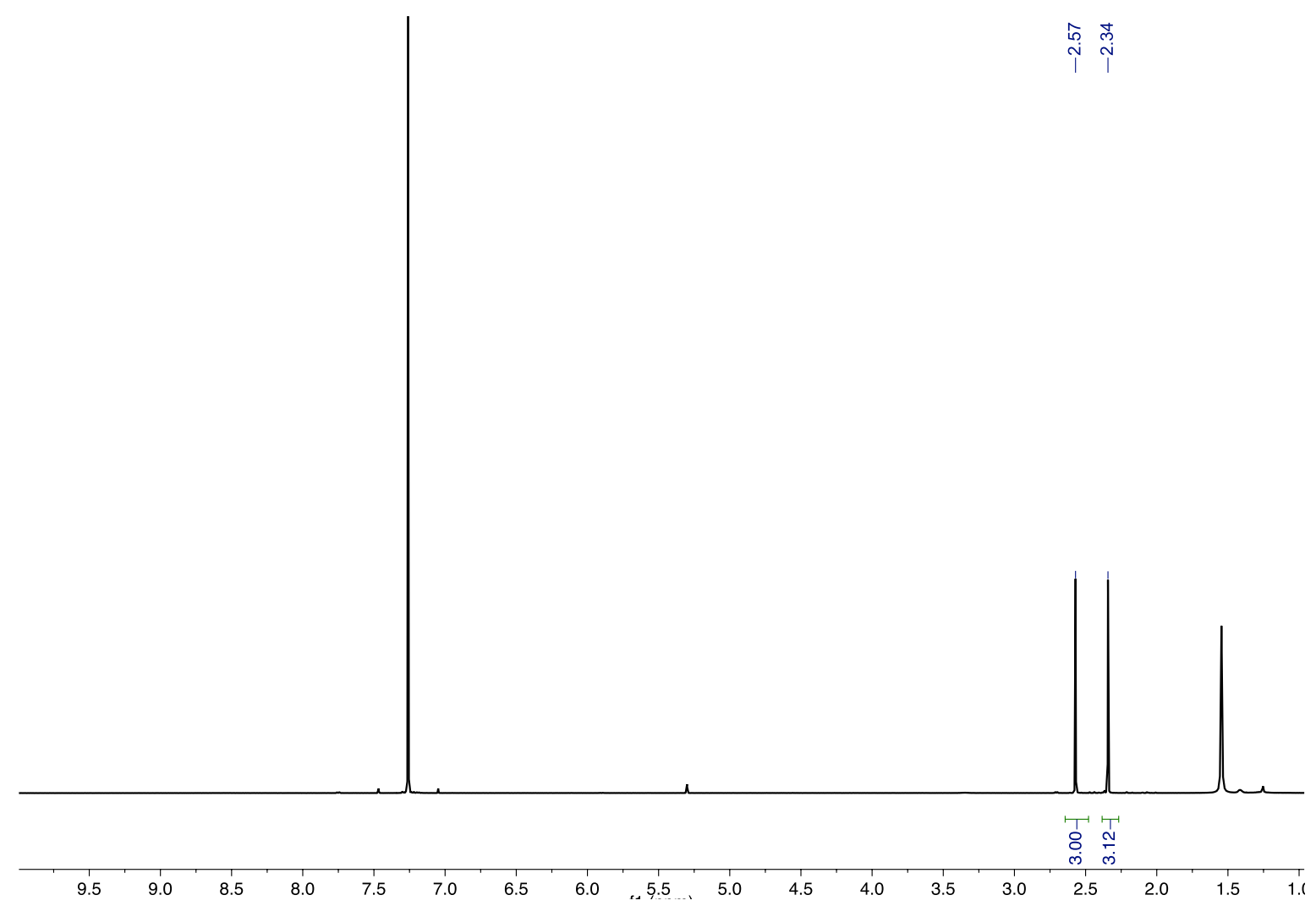

Figure S17. ${ }^{1} \mathrm{H}$ NMR spectrum of 18 in $\mathrm{CDCl}_{3}$.

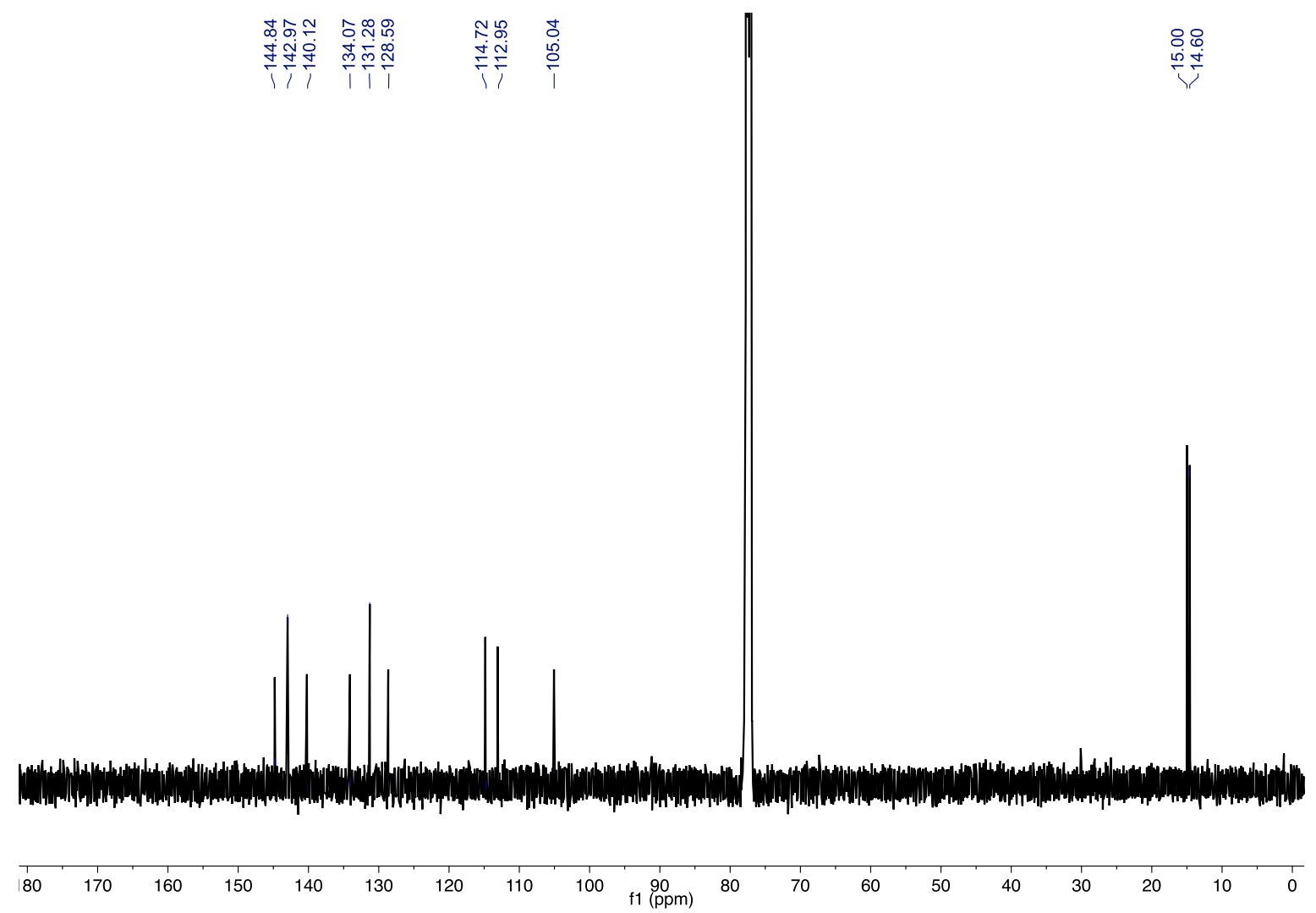

Figure S18. ${ }^{13} \mathrm{C}$ NMR spectrum of 18 in $\mathrm{CDCl}_{3}$. 


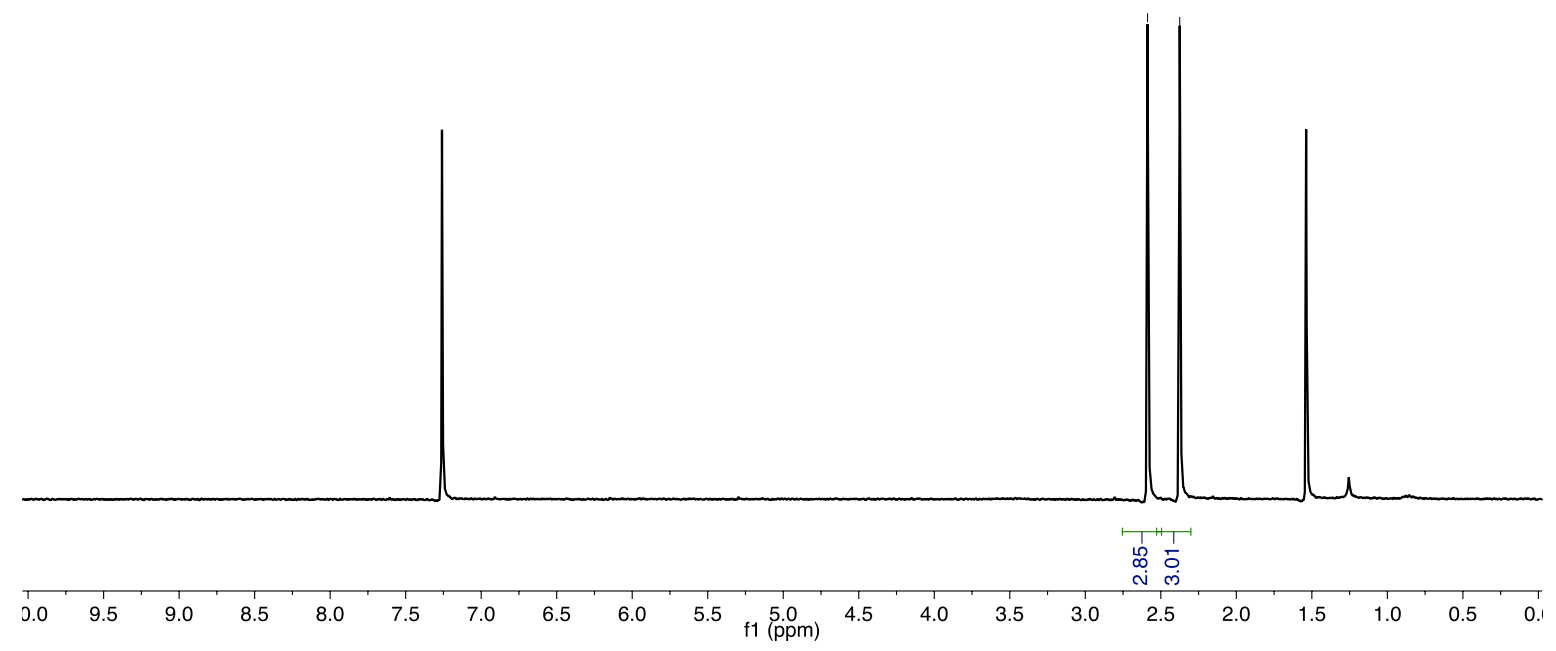

Figure S19. ${ }^{1} \mathrm{H}$ NMR spectrum of 19 in $\mathrm{CDCl}_{3}$.

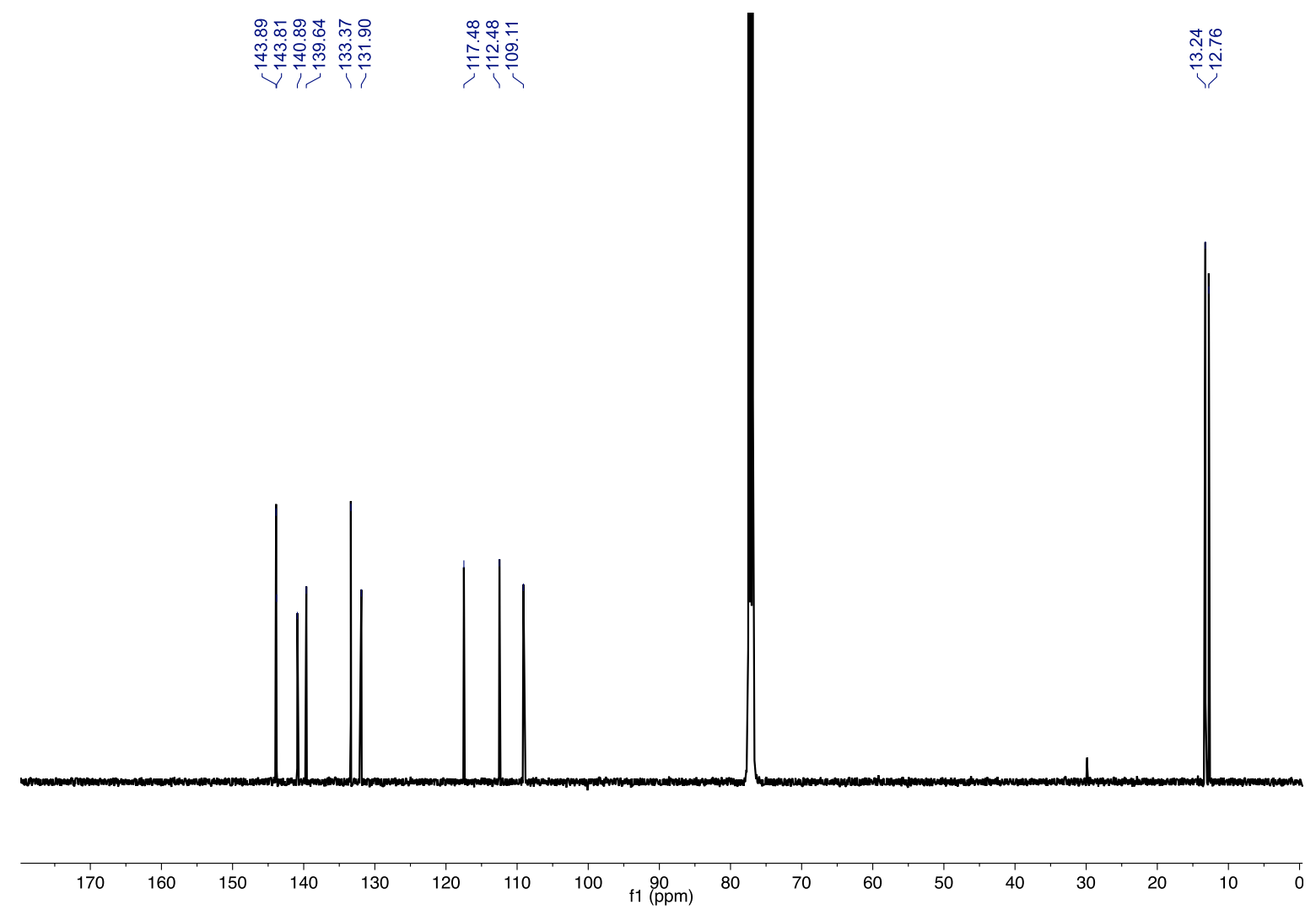

Figure S20. ${ }^{13} \mathrm{C}$ NMR spectrum of 19 in $\mathrm{CDCl}_{3}$. 


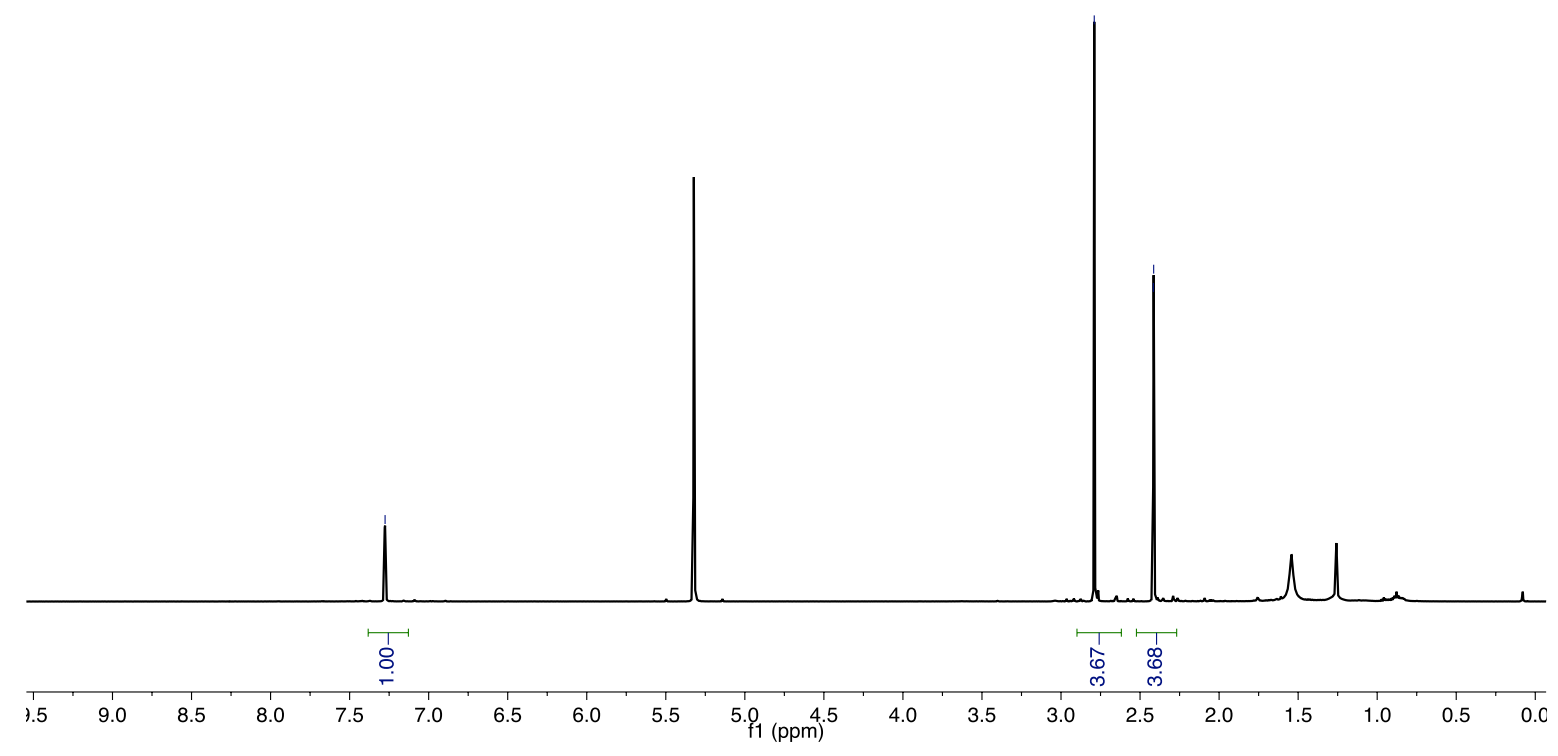

Figure S21. ${ }^{1} \mathrm{H}$ NMR spectrum of 20 in $\mathrm{CDCl}_{3}$.

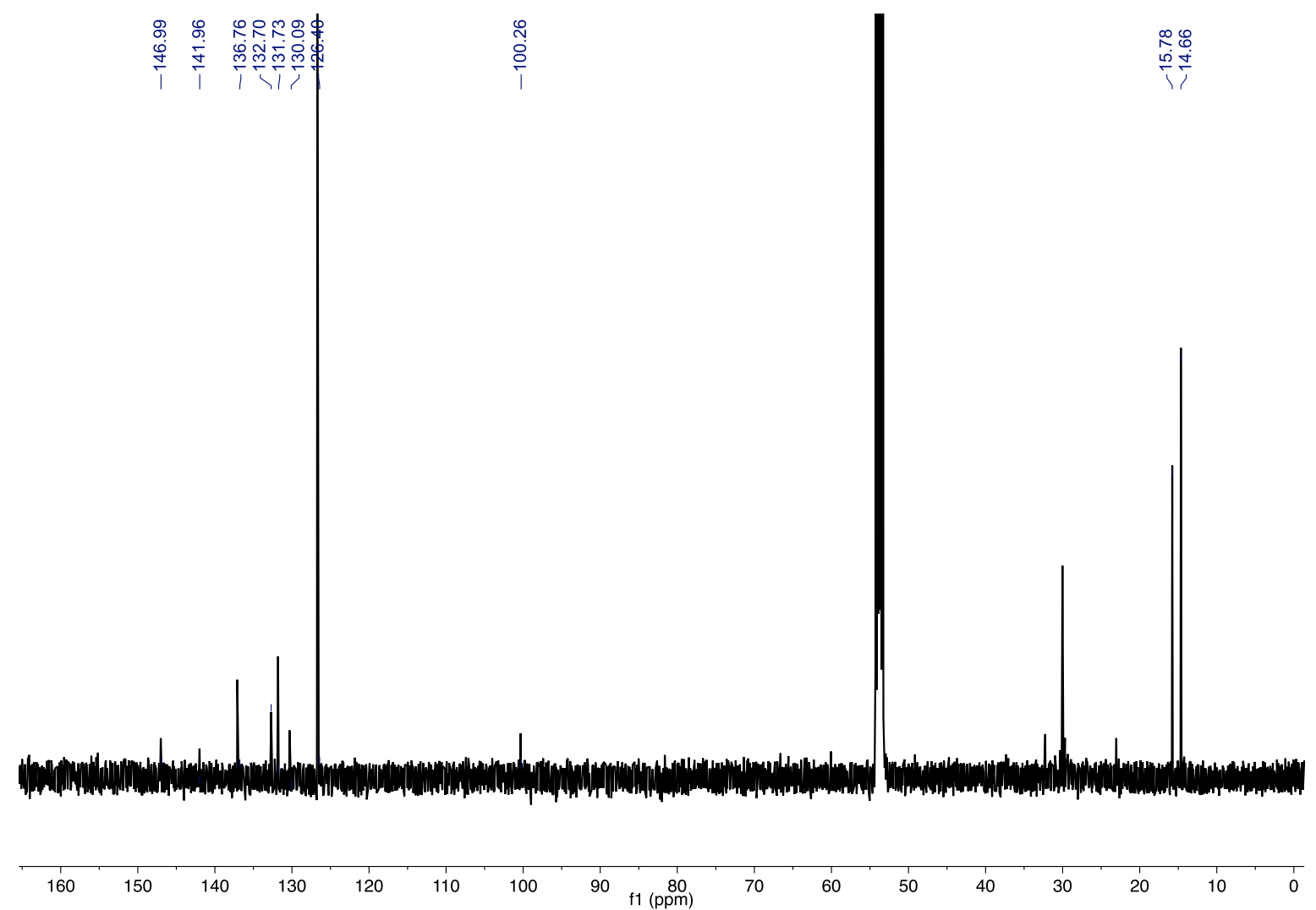

Figure S22. ${ }^{13} \mathrm{C}$ NMR spectrum of 20 in $\mathrm{CDCl}_{3}$ 


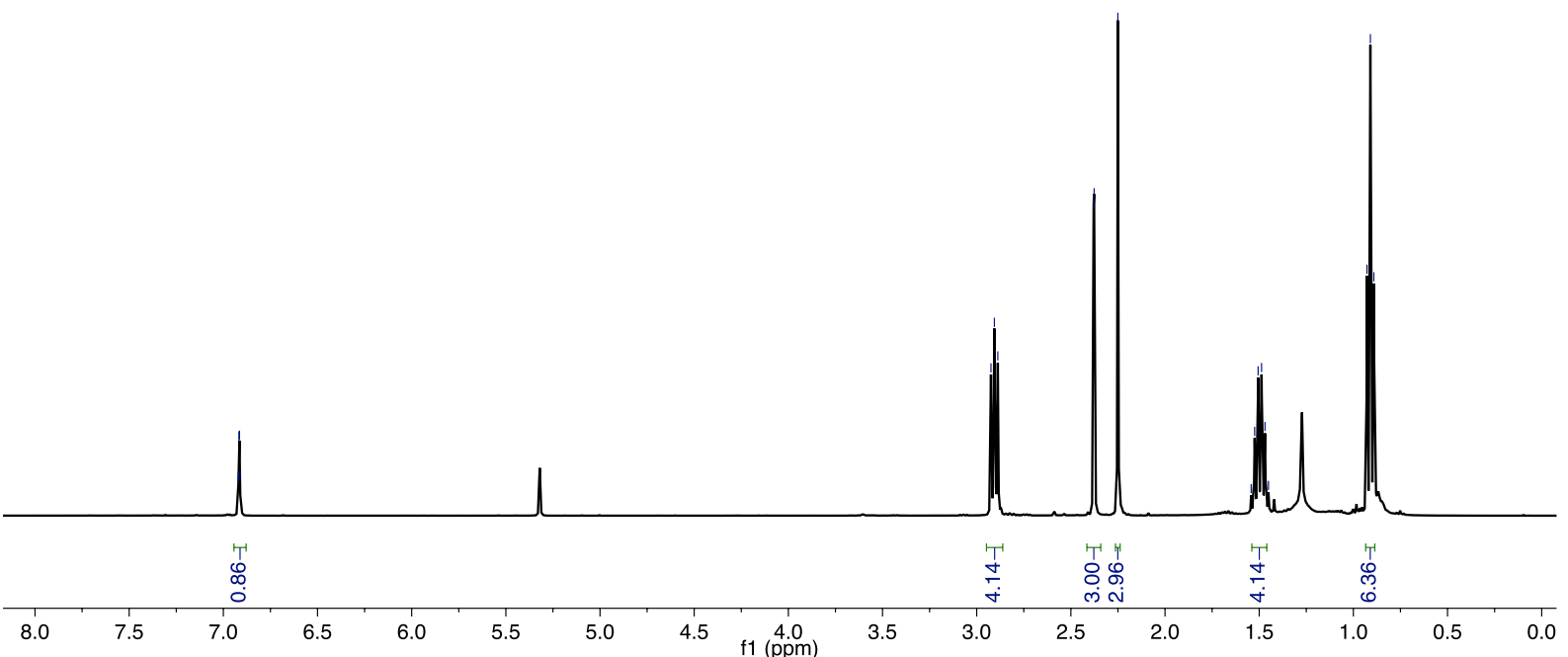

Figure S23. ${ }^{1} \mathrm{H}$ NMR spectrum of 22 in $\mathrm{CD}_{2} \mathrm{Cl}_{2}$.

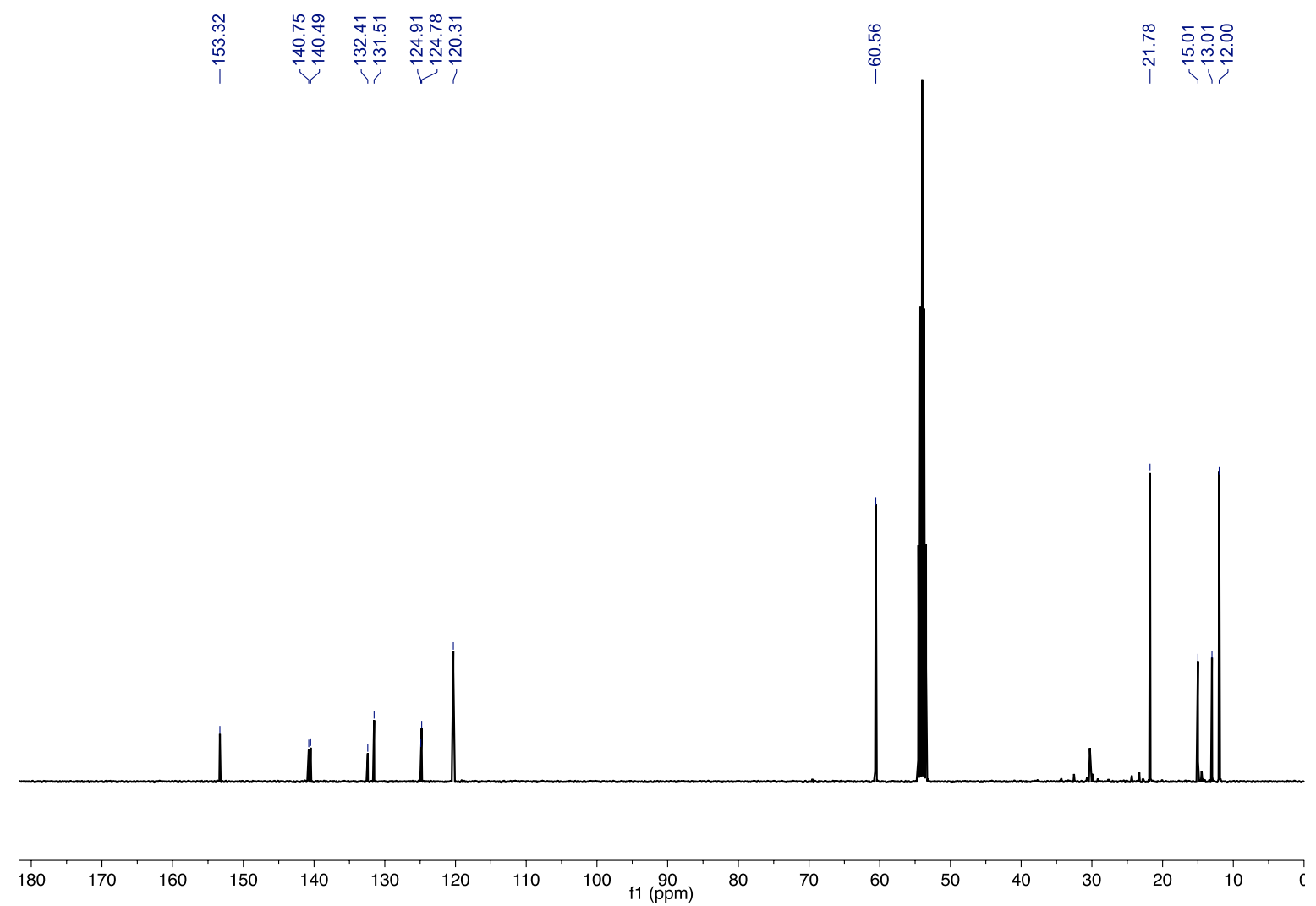

Figure S24. ${ }^{13} \mathrm{C}$ NMR spectrum of 22 in $\mathrm{CD}_{2} \mathrm{Cl}_{2}$. 


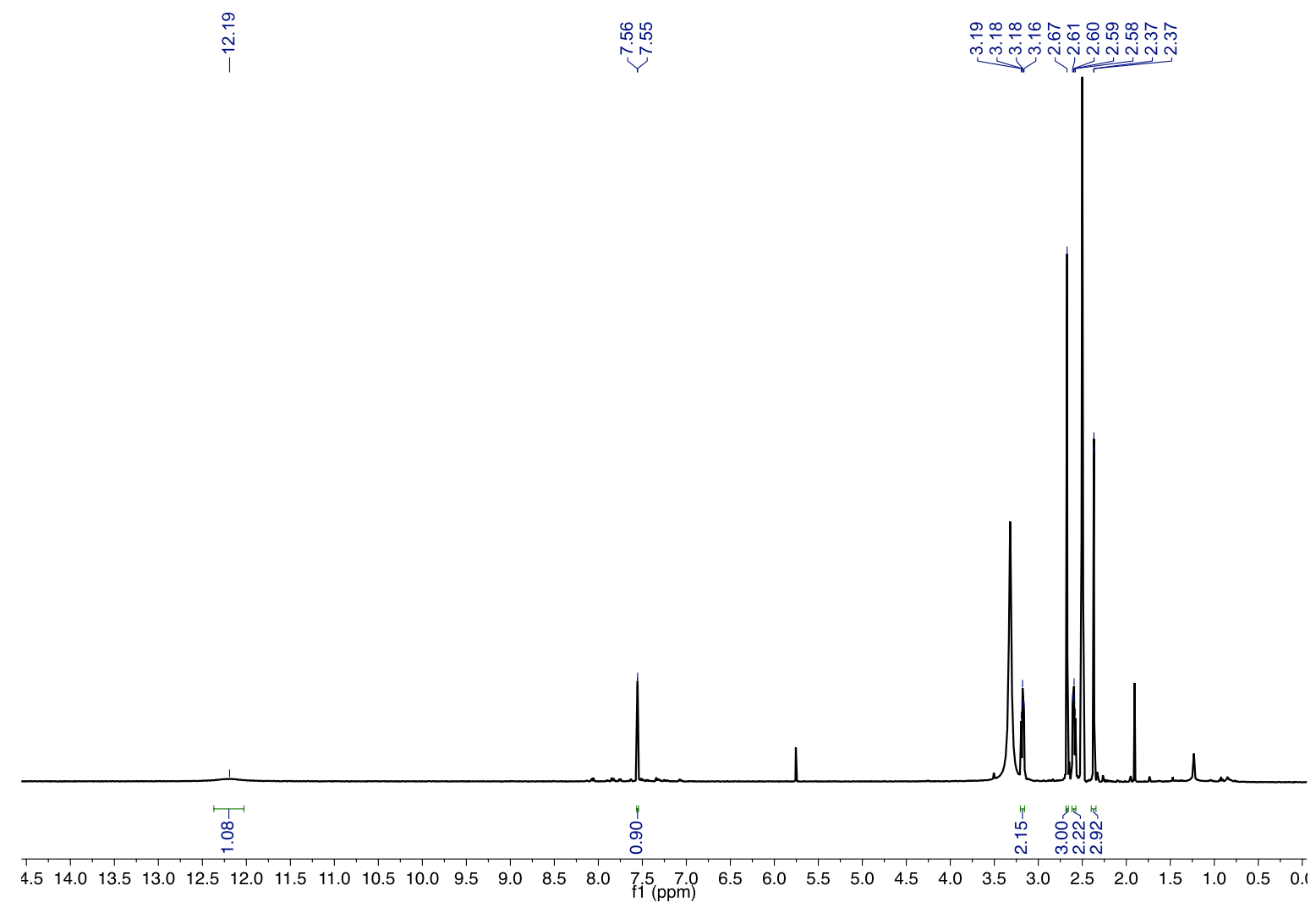

Figure S25. ${ }^{1} \mathrm{H}$ NMR spectrum of 23 in DMSO- $d_{6}$.

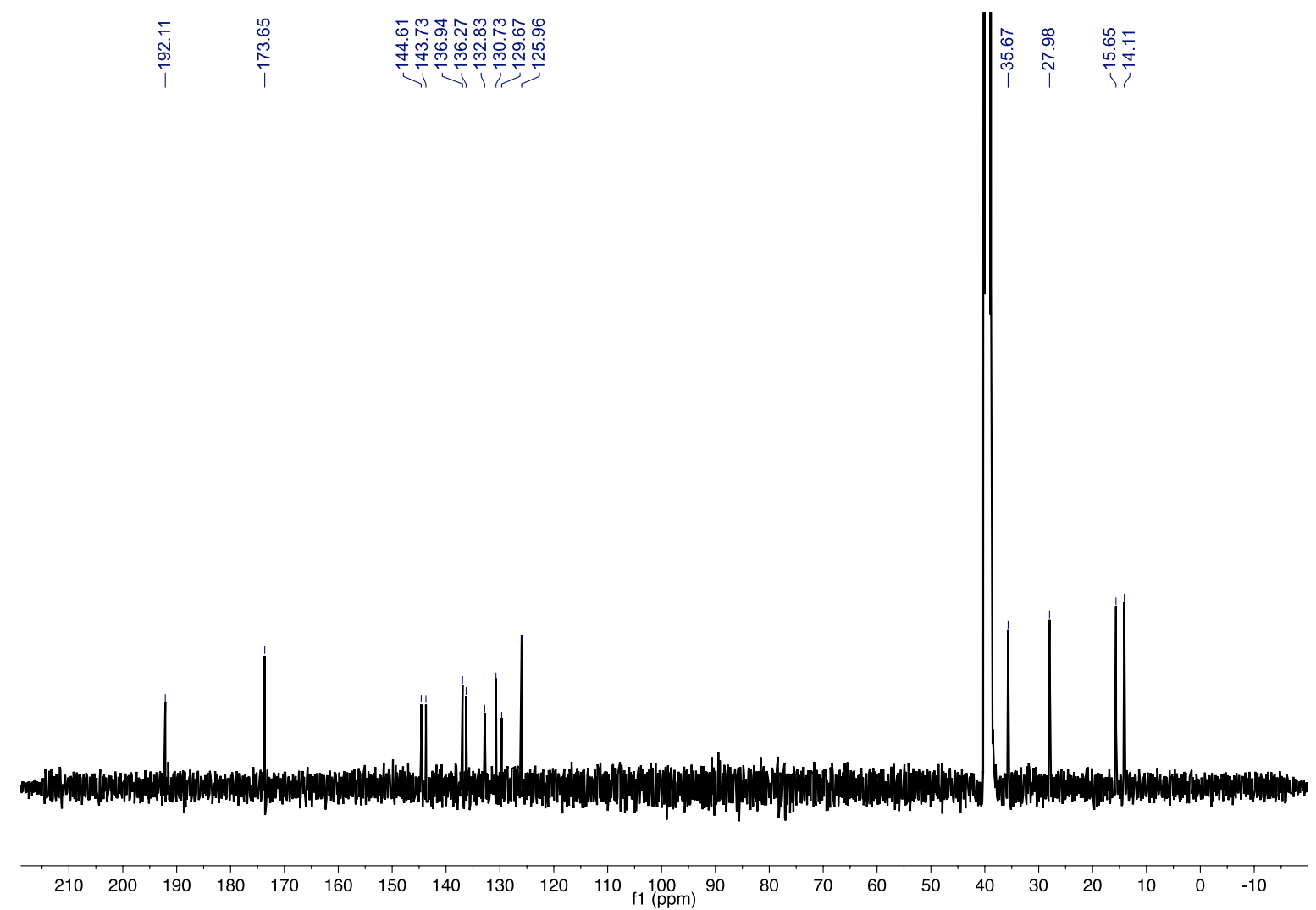

Figure S26. ${ }^{13} \mathrm{C}$ NMR spectrum of 23 in DMSO- $d_{6}$. 


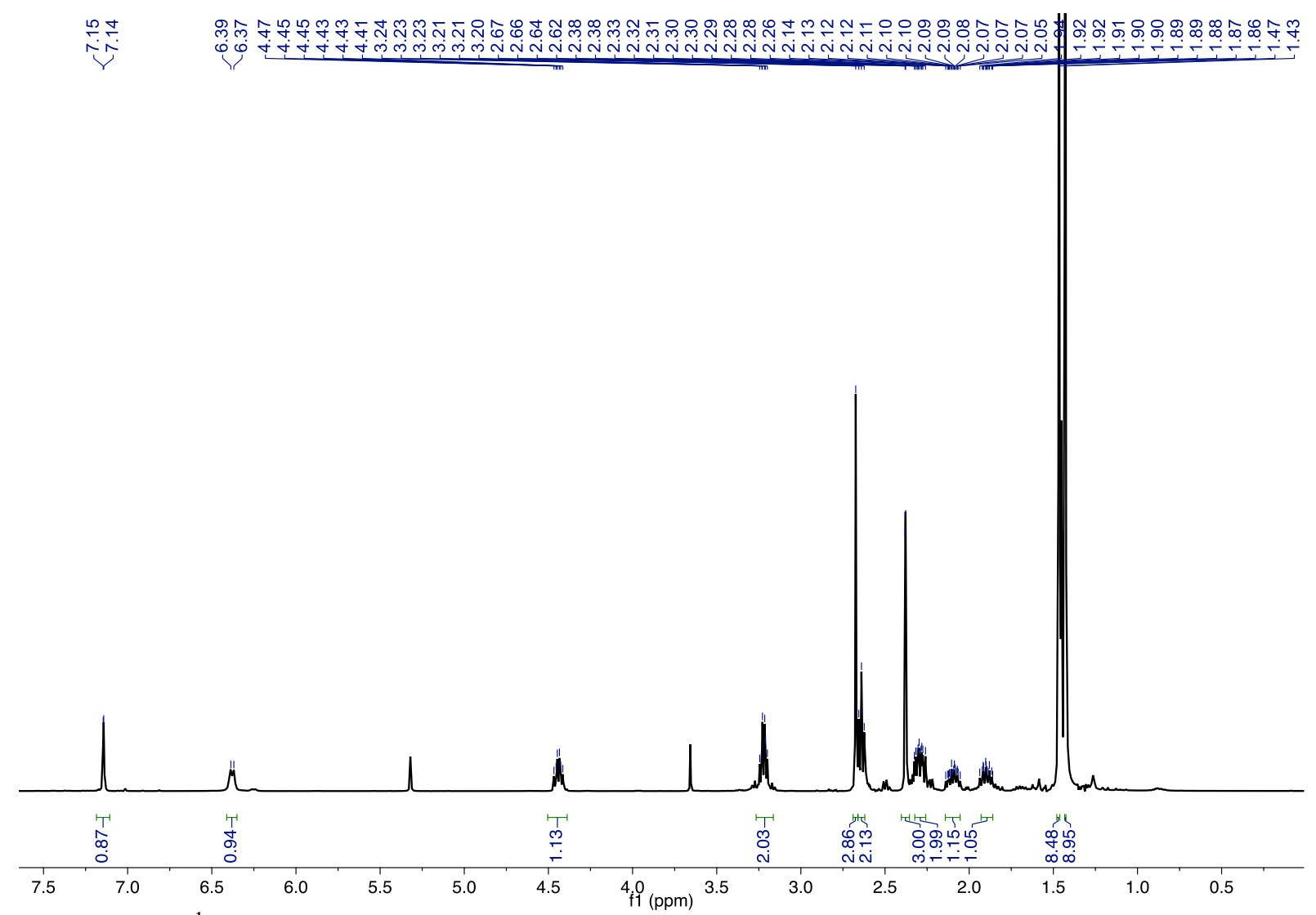

Figure S27. ${ }^{1} \mathrm{H} \mathrm{NMR}$ spectrum of 24 in $\mathrm{CD}_{2} \mathrm{Cl}_{2}$.

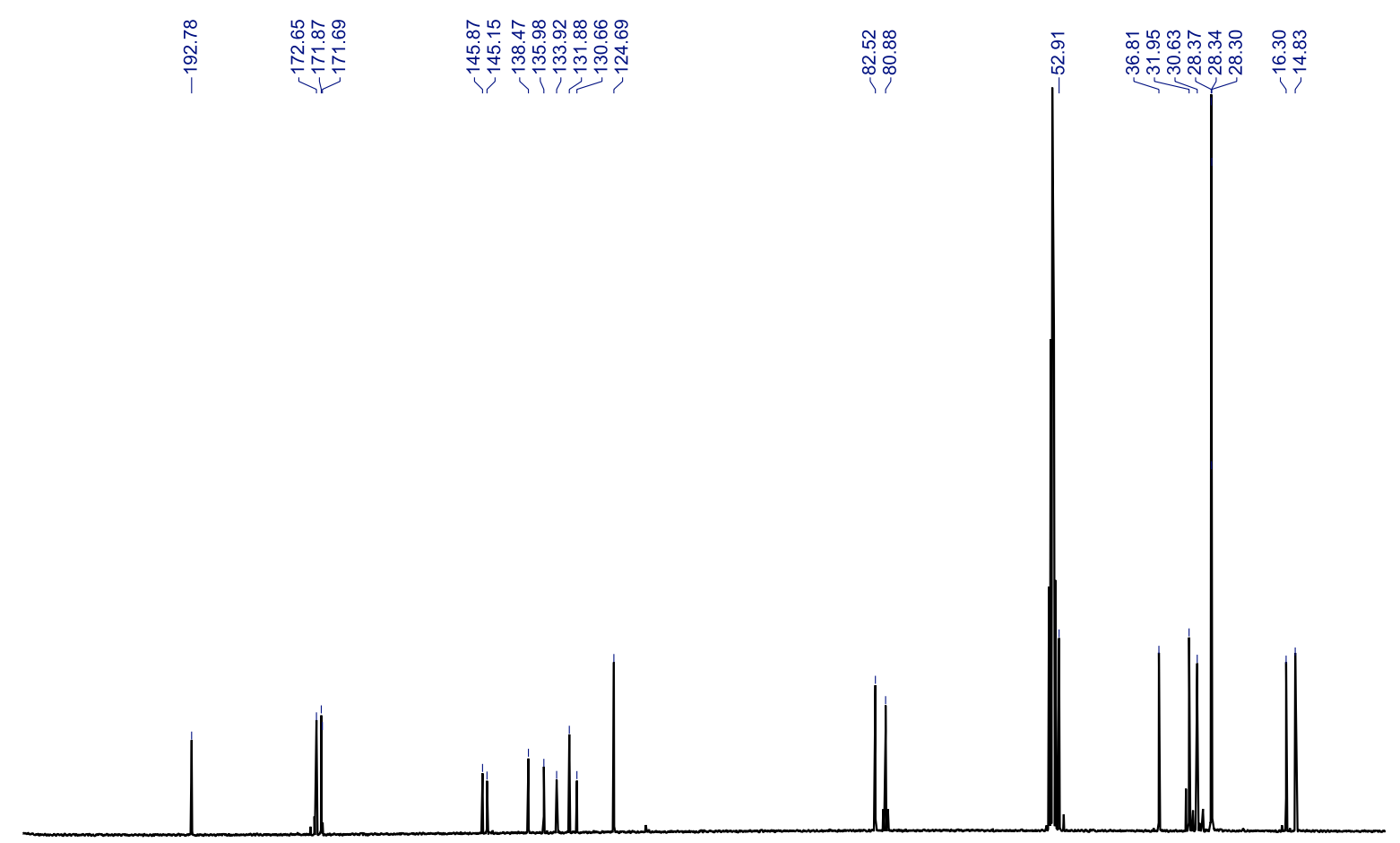

$\begin{array}{lllllllllllllllllll}20 & 210 & 200 & 190 & 180 & 170 & 160 & 150 & 140 & 130 & 120 & 1110 \\ \mathrm{f} 1(\mathrm{ppm}) & 100 & 90 & 80 & 70 & 60 & 50 & 40 & 30 & 20 & 10\end{array}$

Figure S28. ${ }^{13} \mathrm{C}$ NMR spectrum of 24 in $\mathrm{CD}_{2} \mathrm{Cl}_{2}$. 


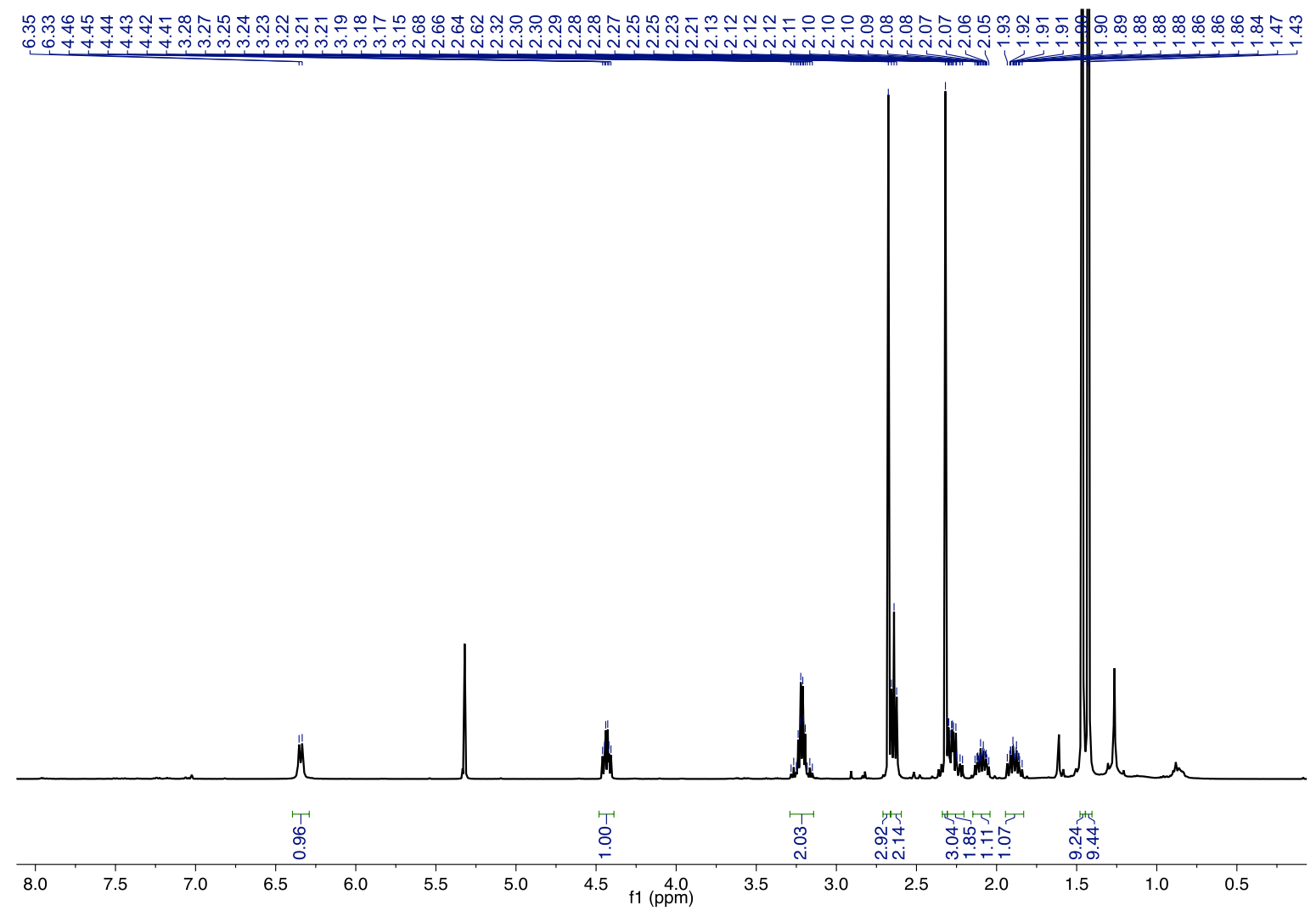

Figure S29. ${ }^{1} \mathrm{H}$ NMR spectrum of 25 in $\mathrm{CD}_{2} \mathrm{Cl}_{2}$.

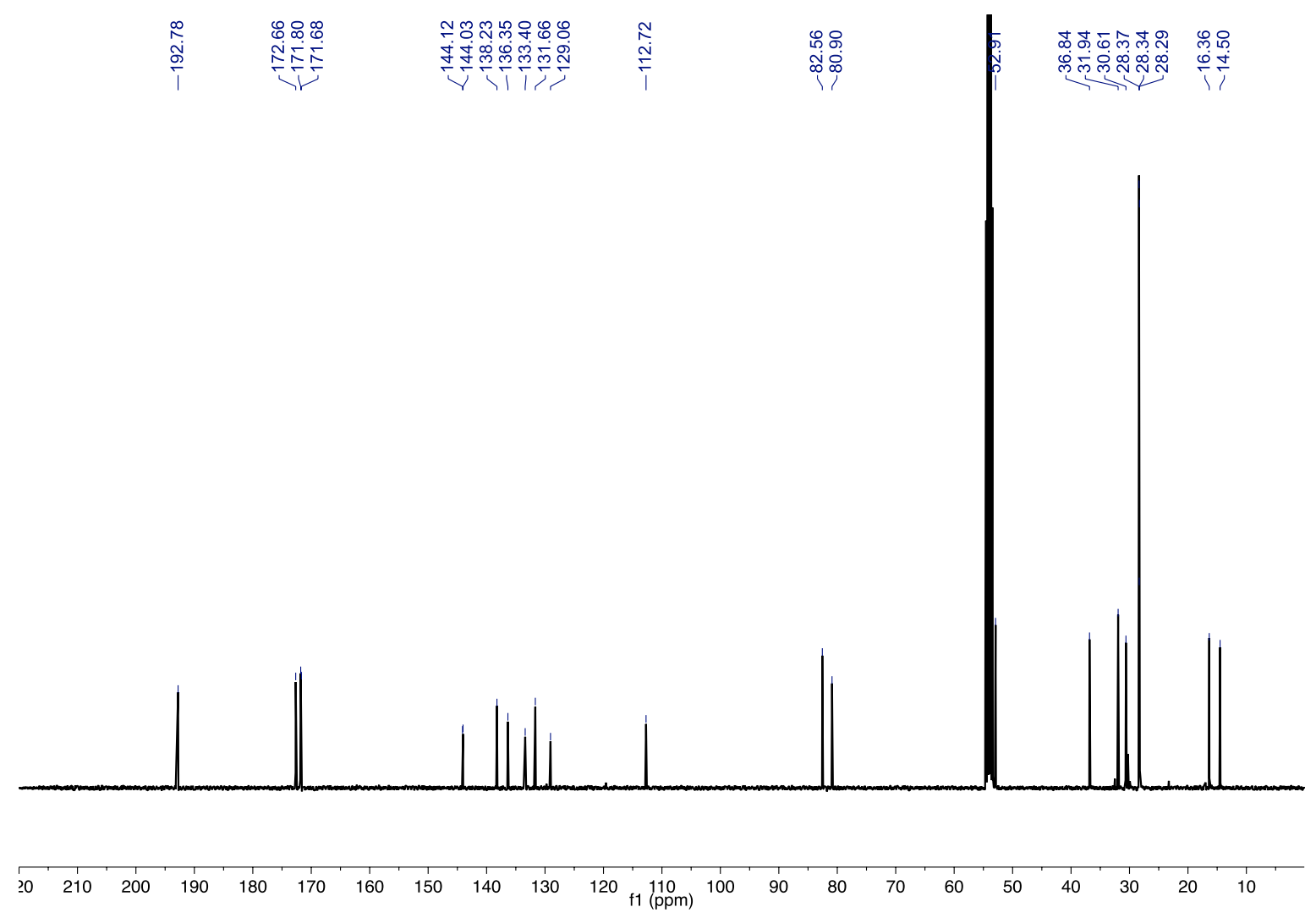

Figure S30. ${ }^{13} \mathrm{C}$ NMR spectrum of 25 in $\mathrm{CD}_{2} \mathrm{Cl}_{2}$. 


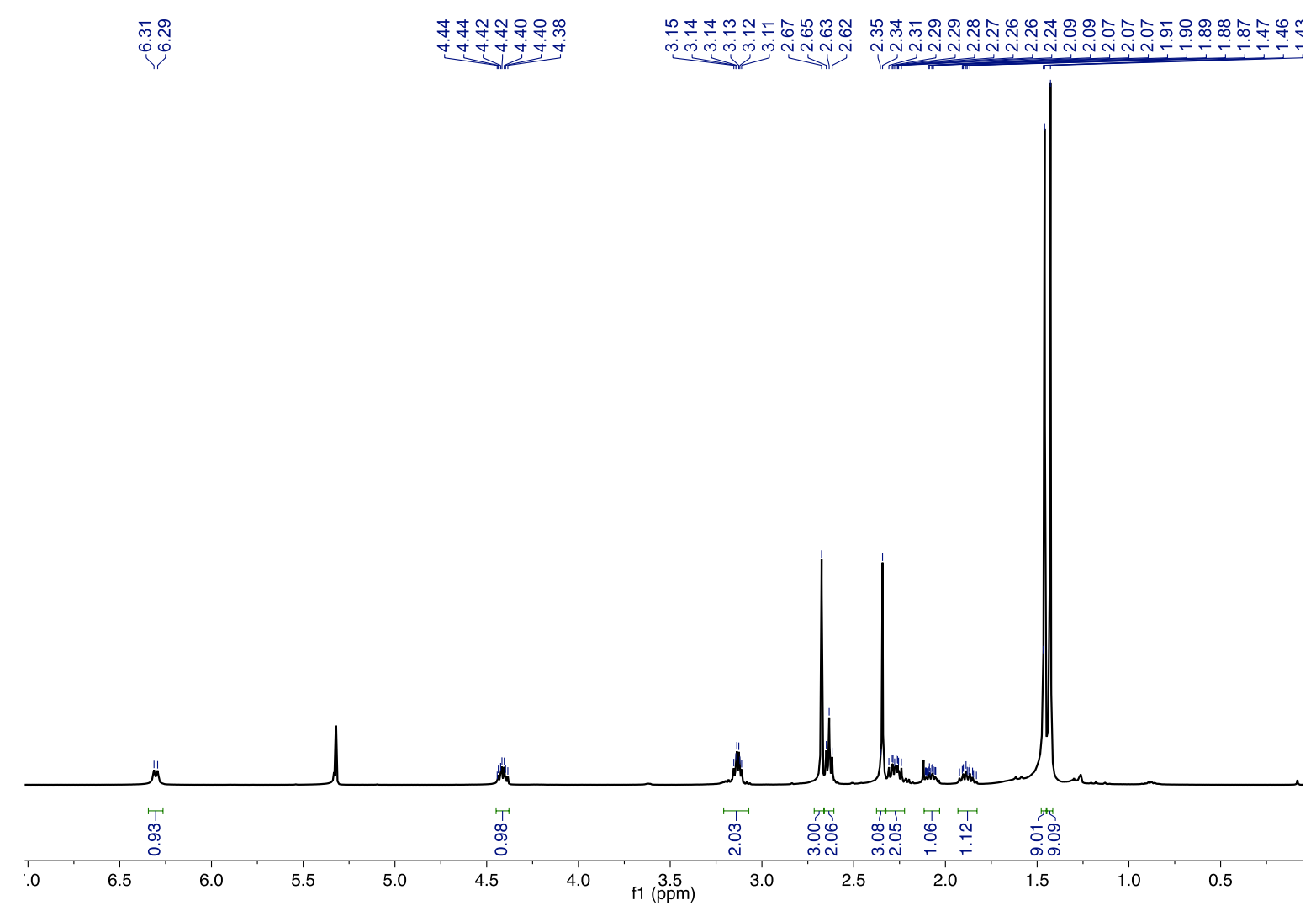

Figure S31. ${ }^{1} \mathrm{H}$ NMR spectrum of 26 in $\mathrm{CD}_{2} \mathrm{Cl}_{2}$.

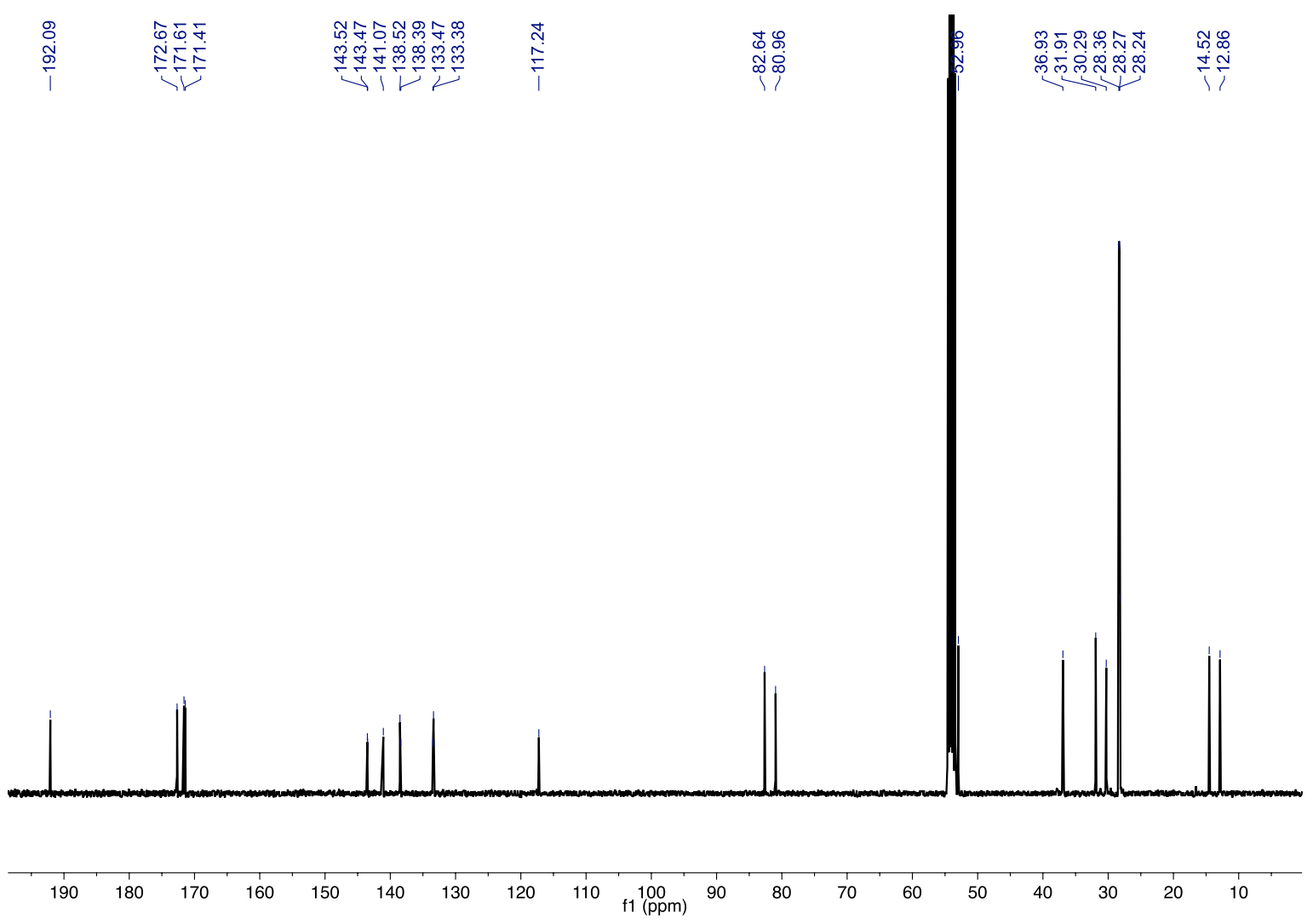

Figure S32. ${ }^{13} \mathrm{C}$ NMR spectrum of 26 in $\mathrm{CD}_{2} \mathrm{Cl}_{2}$. 


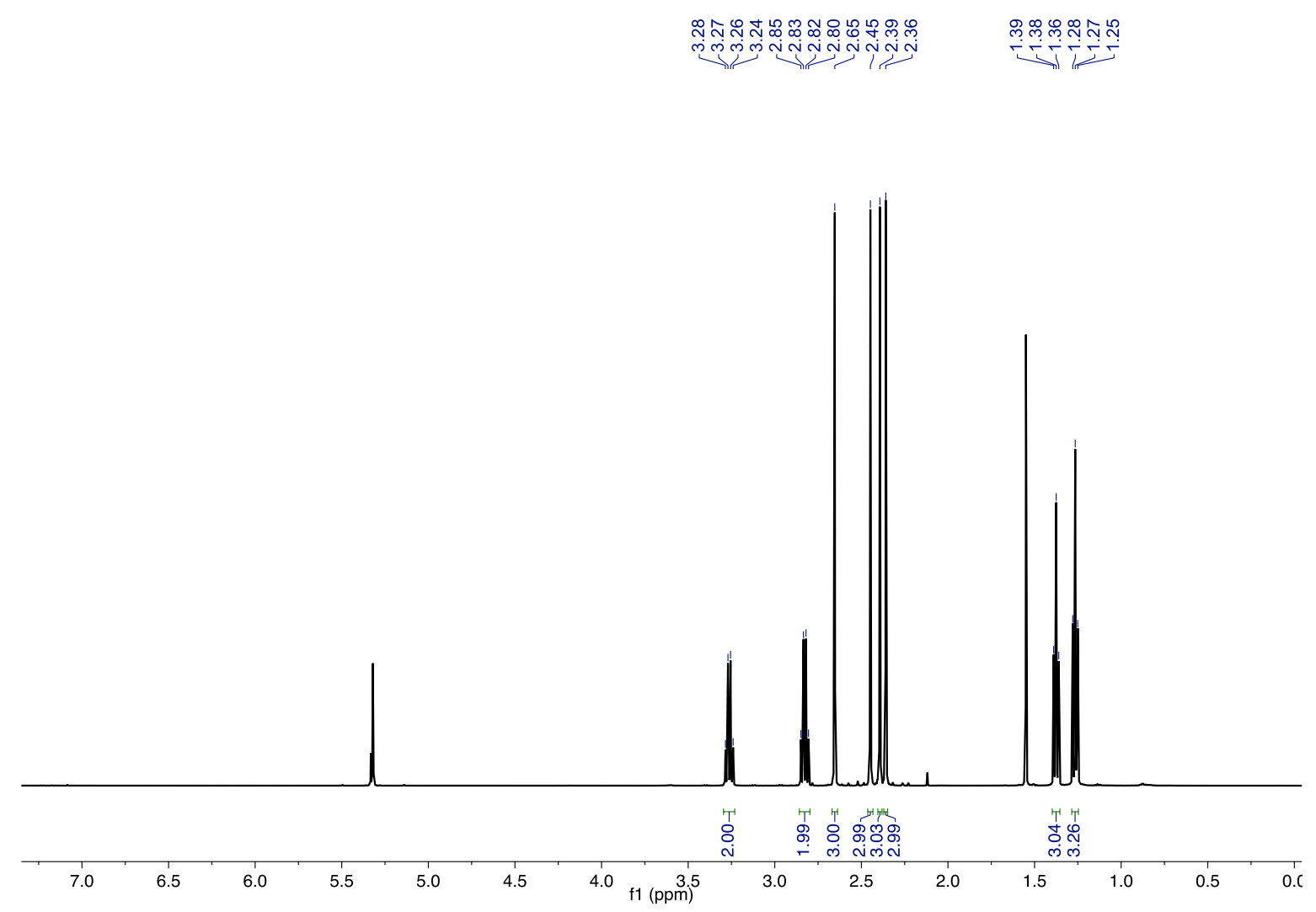

Figure S33. ${ }^{1} \mathrm{H}$ NMR spectrum of 2 in $\mathrm{CD}_{2} \mathrm{Cl}_{2}$.

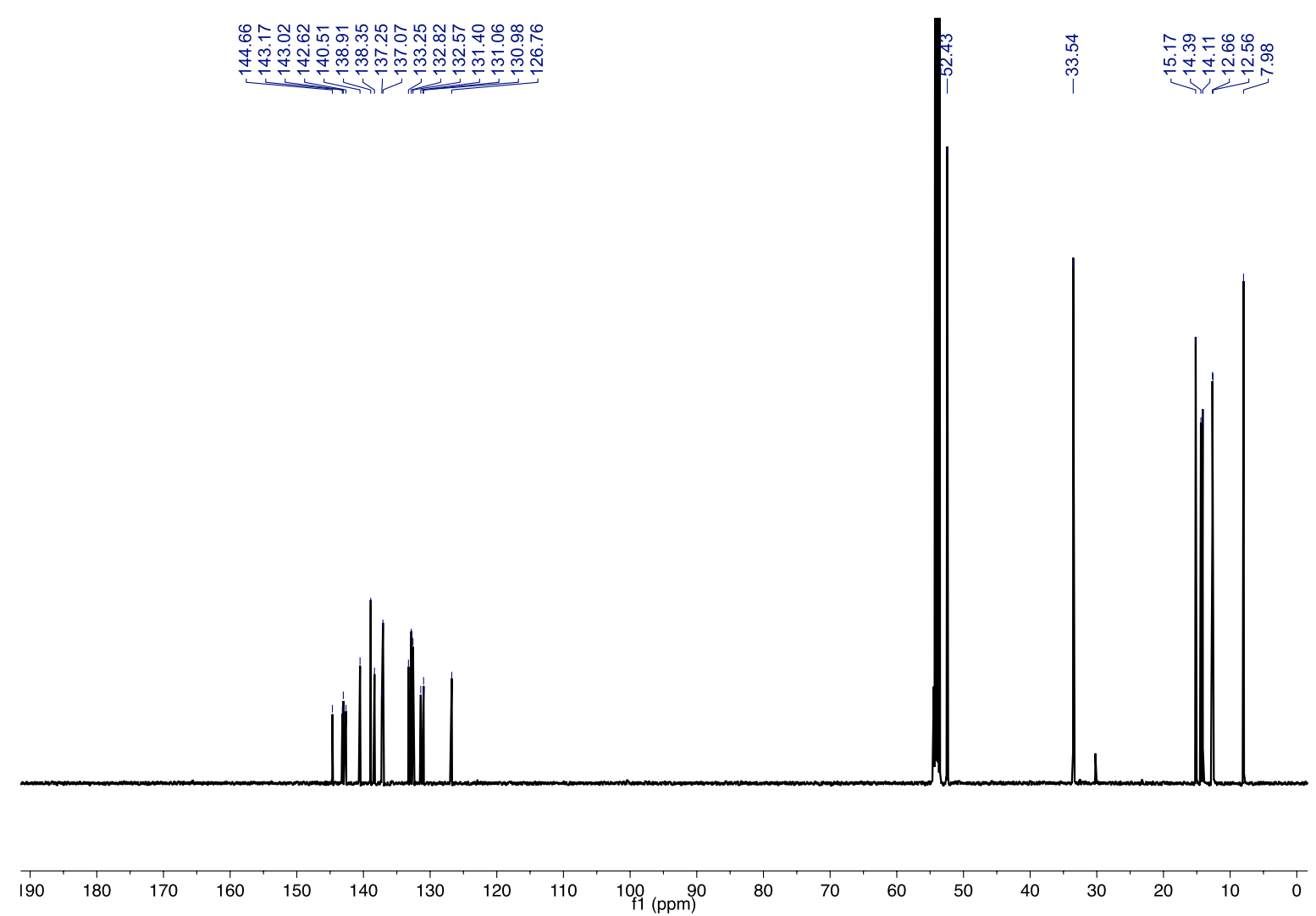

Figure S34. ${ }^{13} \mathrm{C}$ NMR spectrum of 2 in $\mathrm{CD}_{2} \mathrm{Cl}_{2}$. 


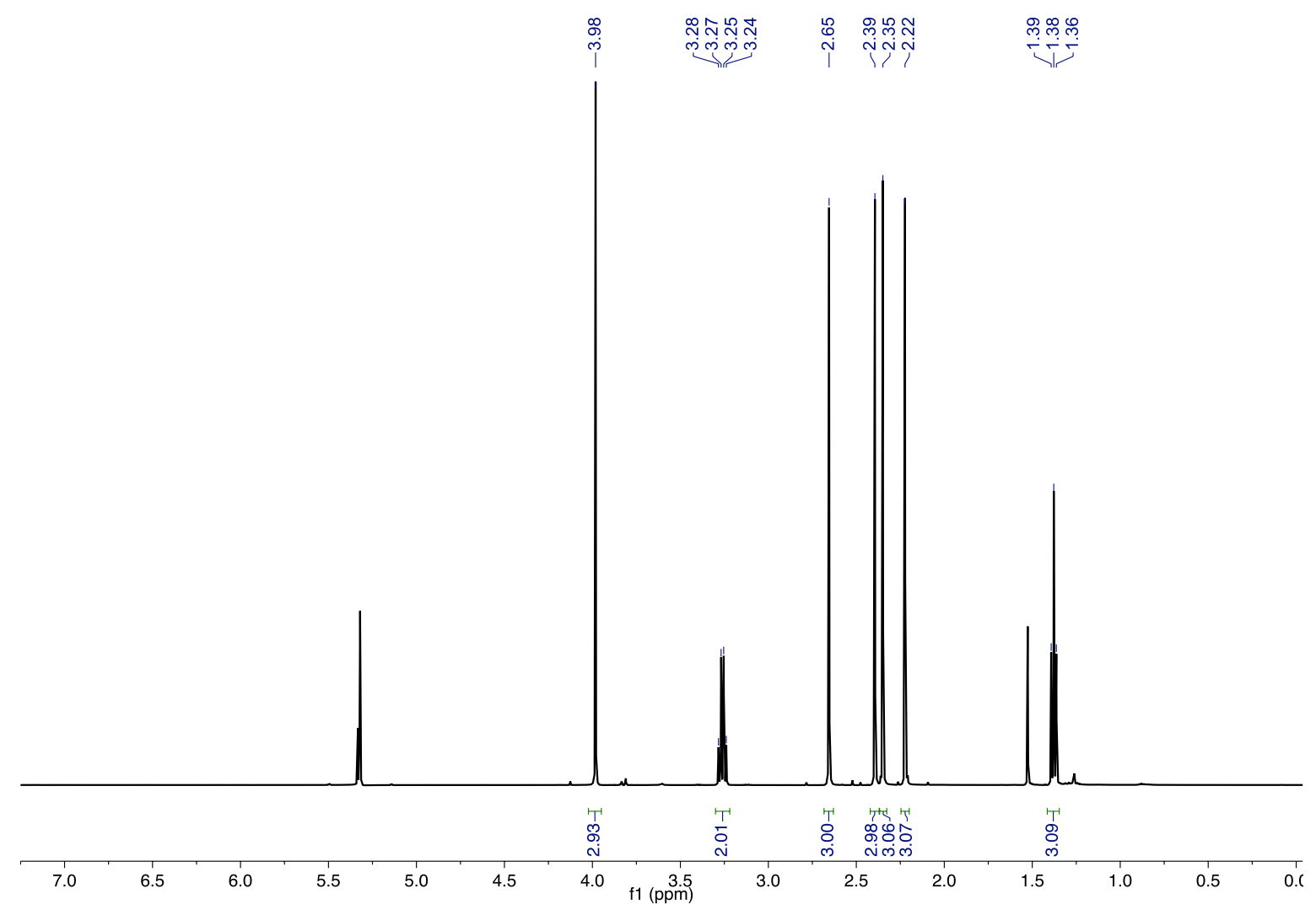

Figure S35. ${ }^{1} \mathrm{H}$ NMR spectrum of 3 in $\mathrm{CD}_{2} \mathrm{Cl}_{2}$.

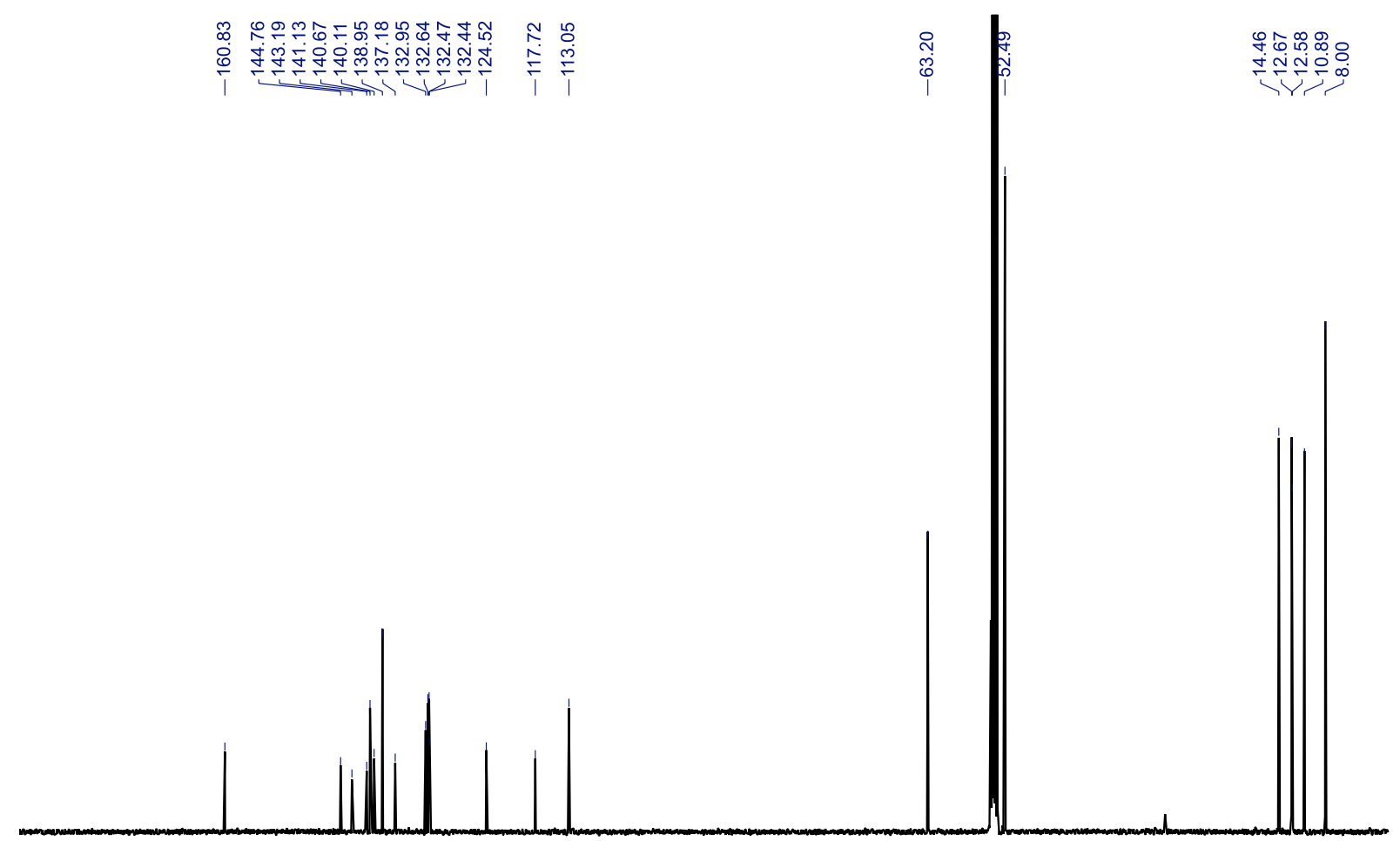

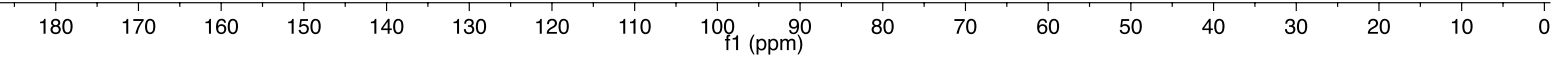

Figure S36. ${ }^{13} \mathrm{C}$ NMR spectrum of 3 in $\mathrm{CD}_{2} \mathrm{Cl}_{2}$. 


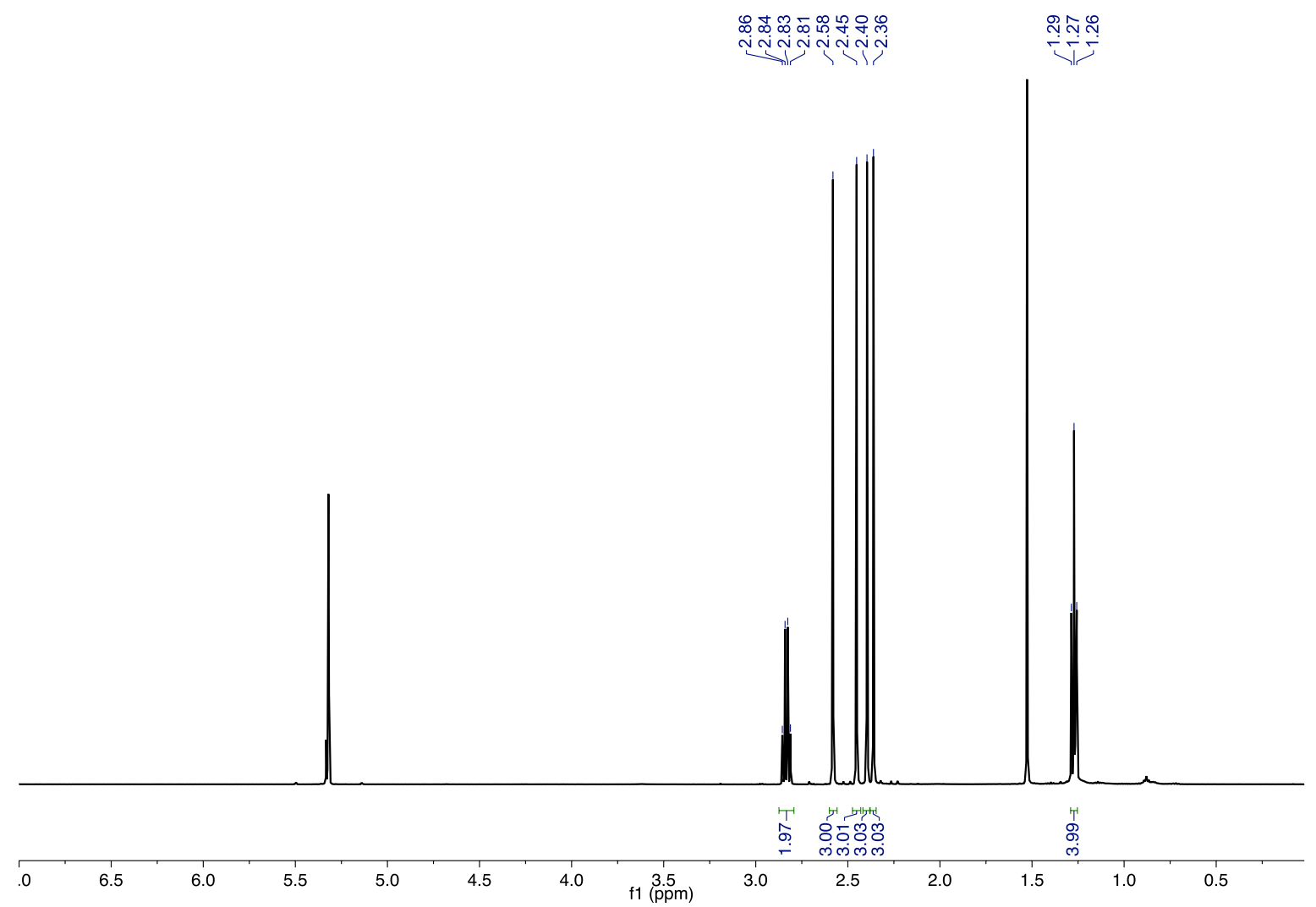

Figure S37. ${ }^{1} \mathrm{H}$ NMR spectrum of 4 in $\mathrm{CD}_{2} \mathrm{Cl}_{2}$.

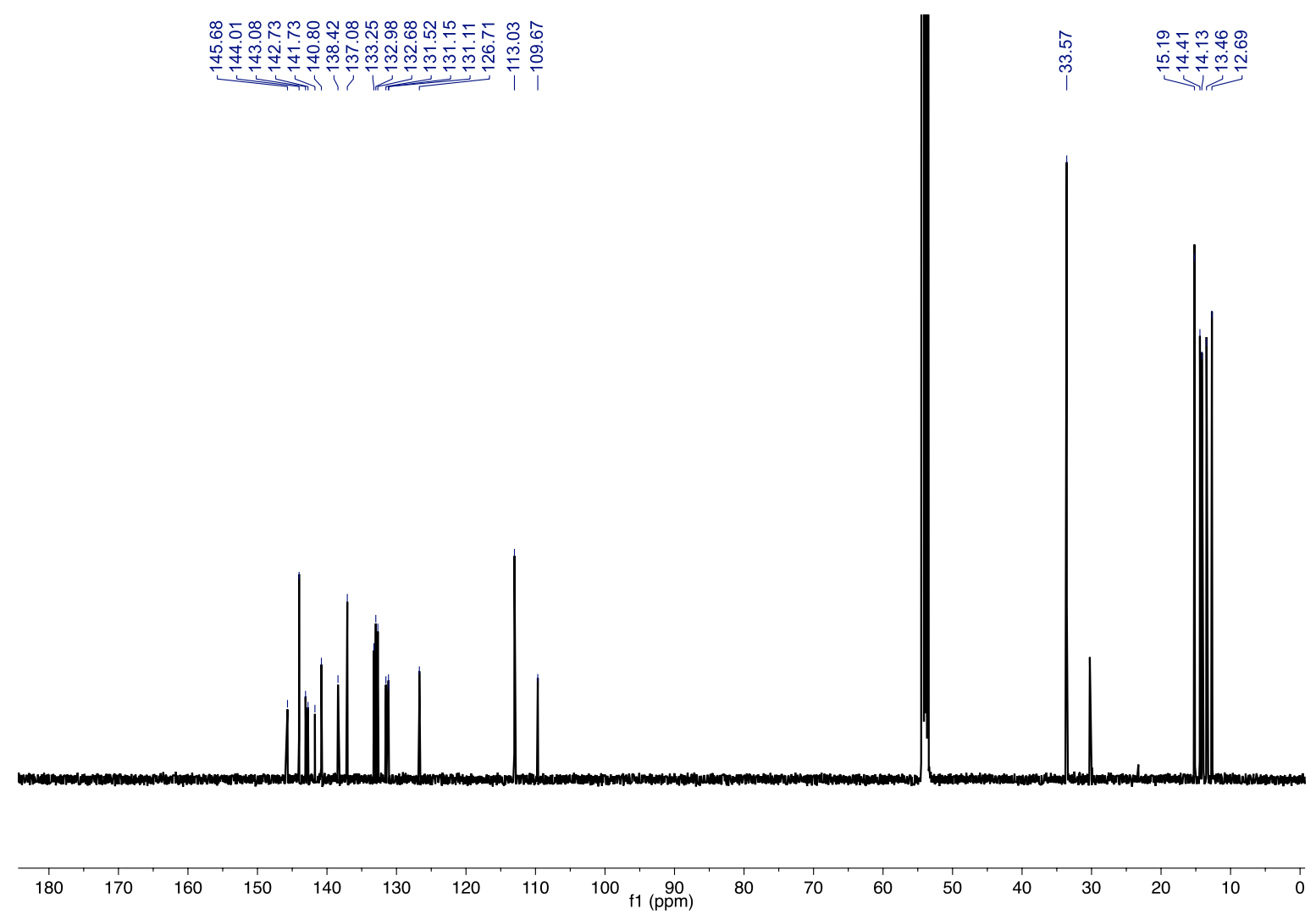

Figure S38. ${ }^{13} \mathrm{C}$ NMR spectrum of 4 in $\mathrm{CD}_{2} \mathrm{Cl}_{2}$. 


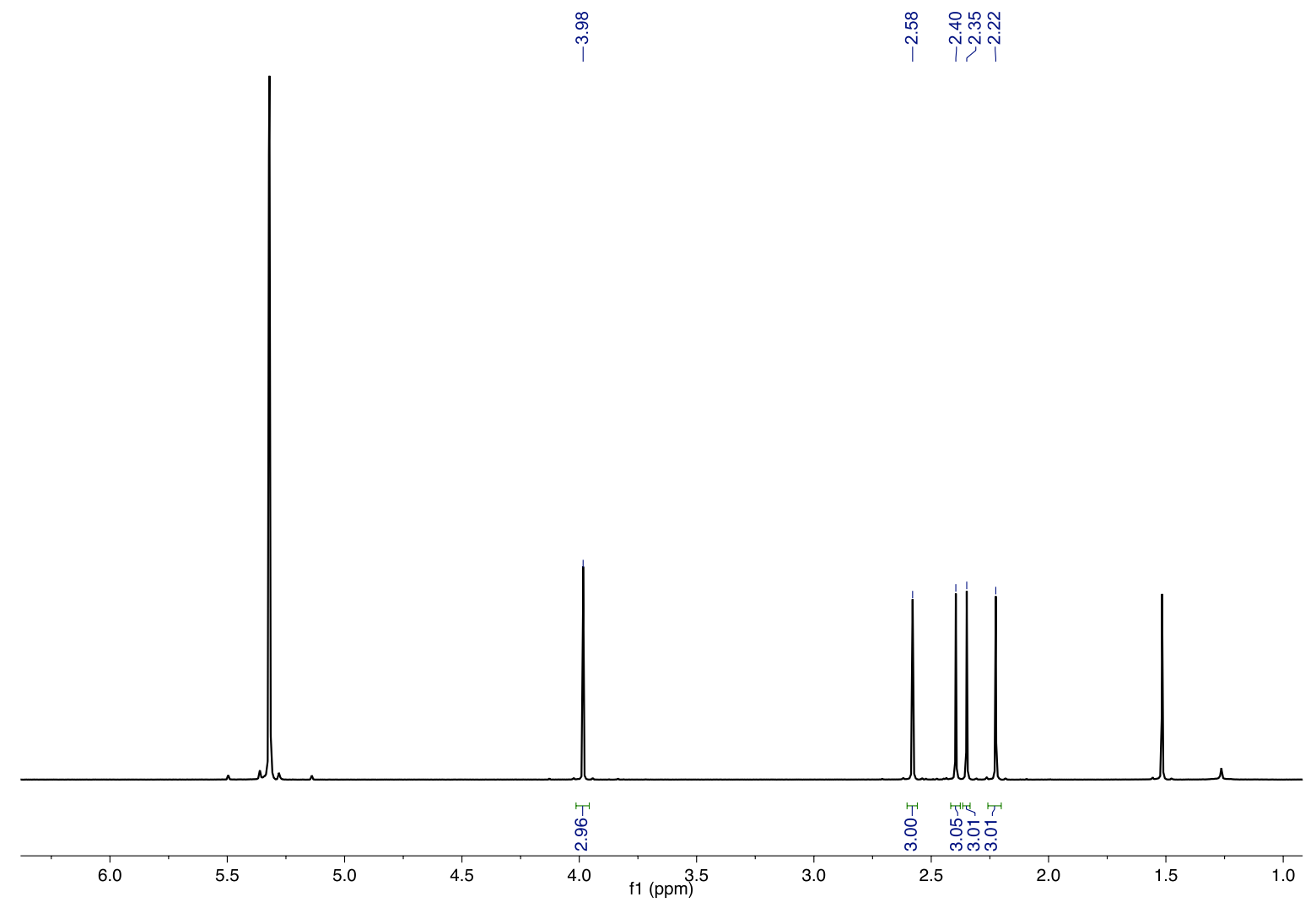

Figure S39. ${ }^{1} \mathrm{H}$ NMR spectrum of 5 in $\mathrm{CD}_{2} \mathrm{Cl}_{2}$.

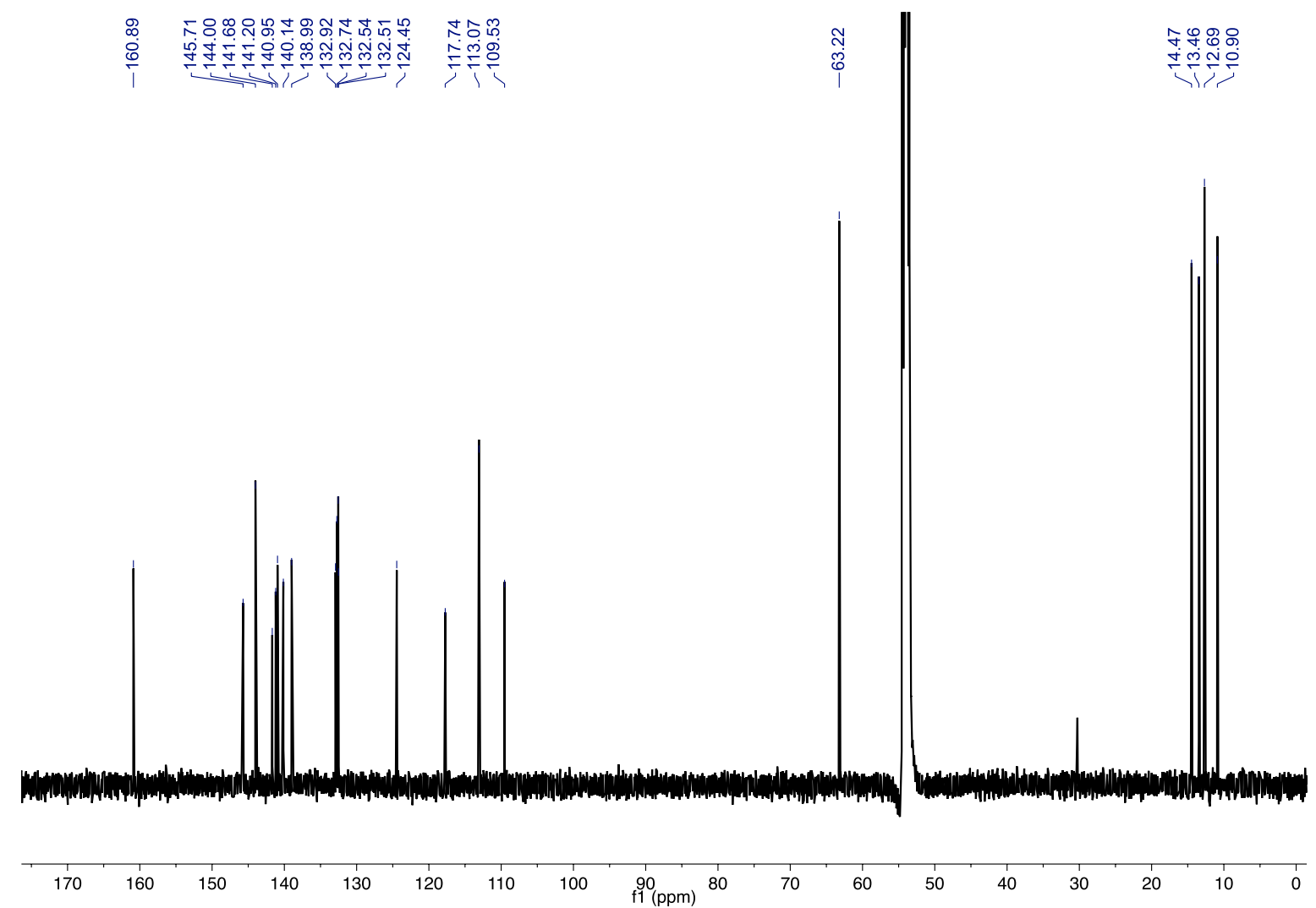

Figure S40. ${ }^{13} \mathrm{C}$ NMR spectrum of 5 in $\mathrm{CD}_{2} \mathrm{Cl}_{2}$. 


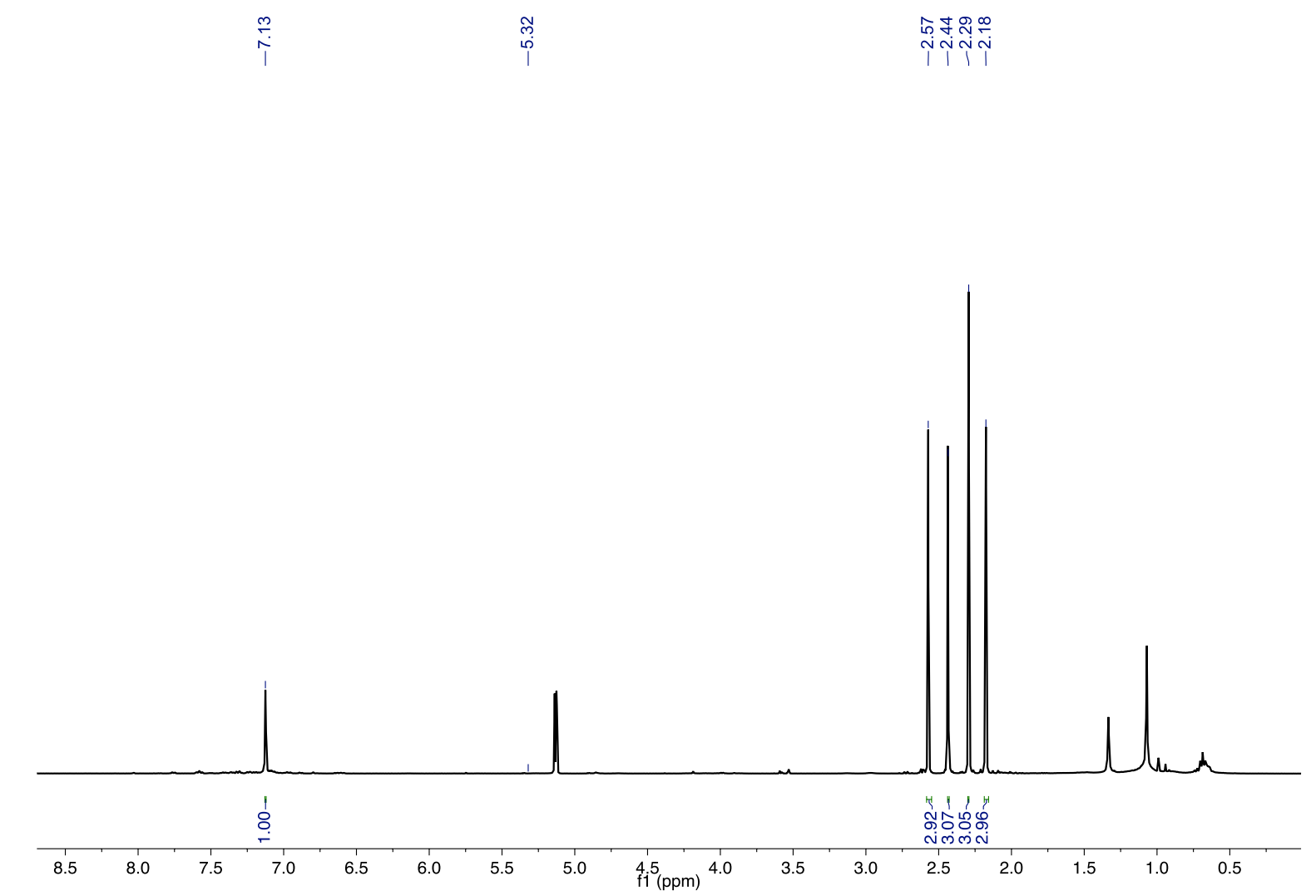

Figure S41. ${ }^{1} \mathrm{H}$ NMR spectrum of 6 in $\mathrm{CD}_{2} \mathrm{Cl}_{2}$.
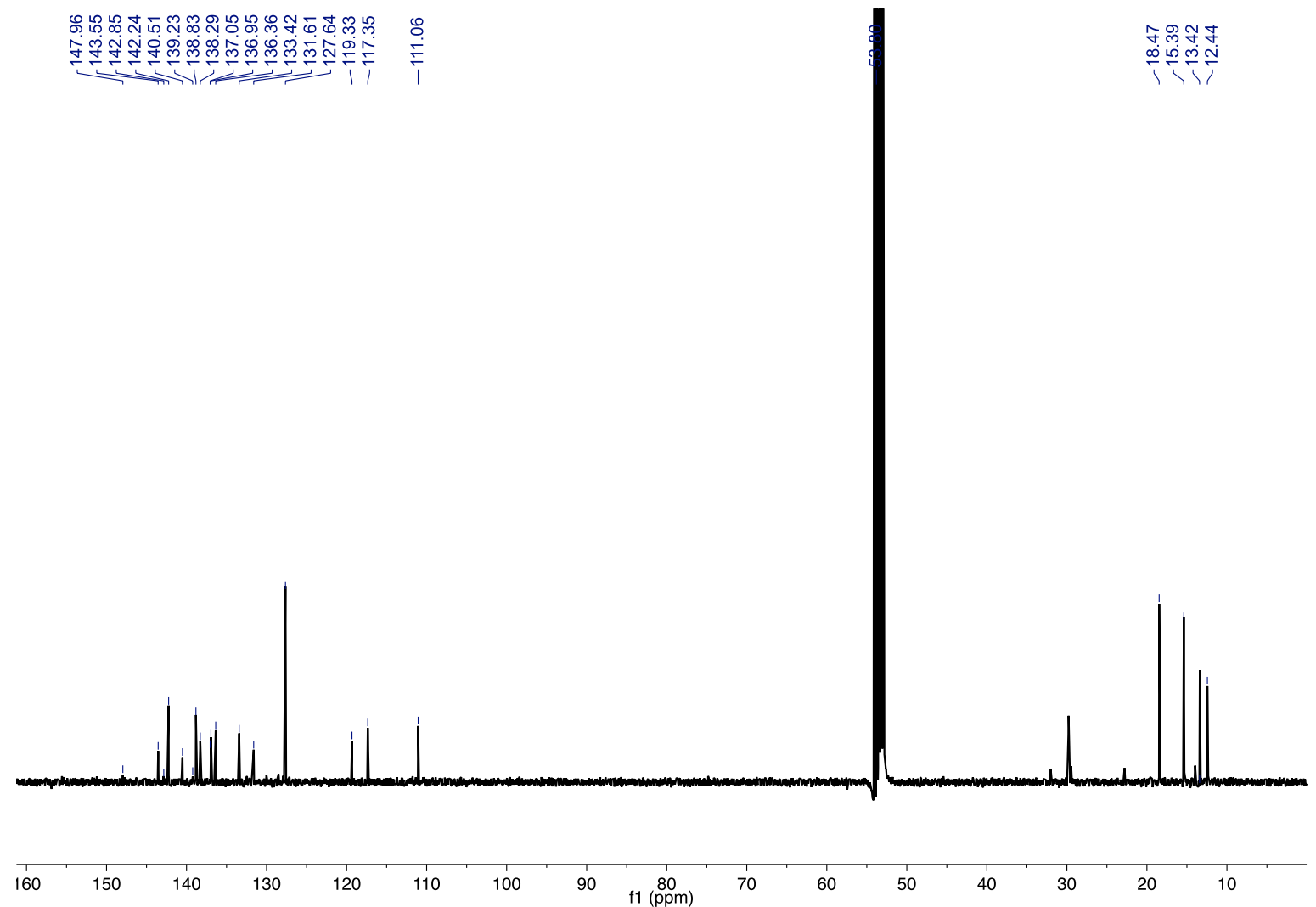

Figure S42. ${ }^{13} \mathrm{C}$ NMR spectrum of 6 in $\mathrm{CD}_{2} \mathrm{Cl}_{2}$. 


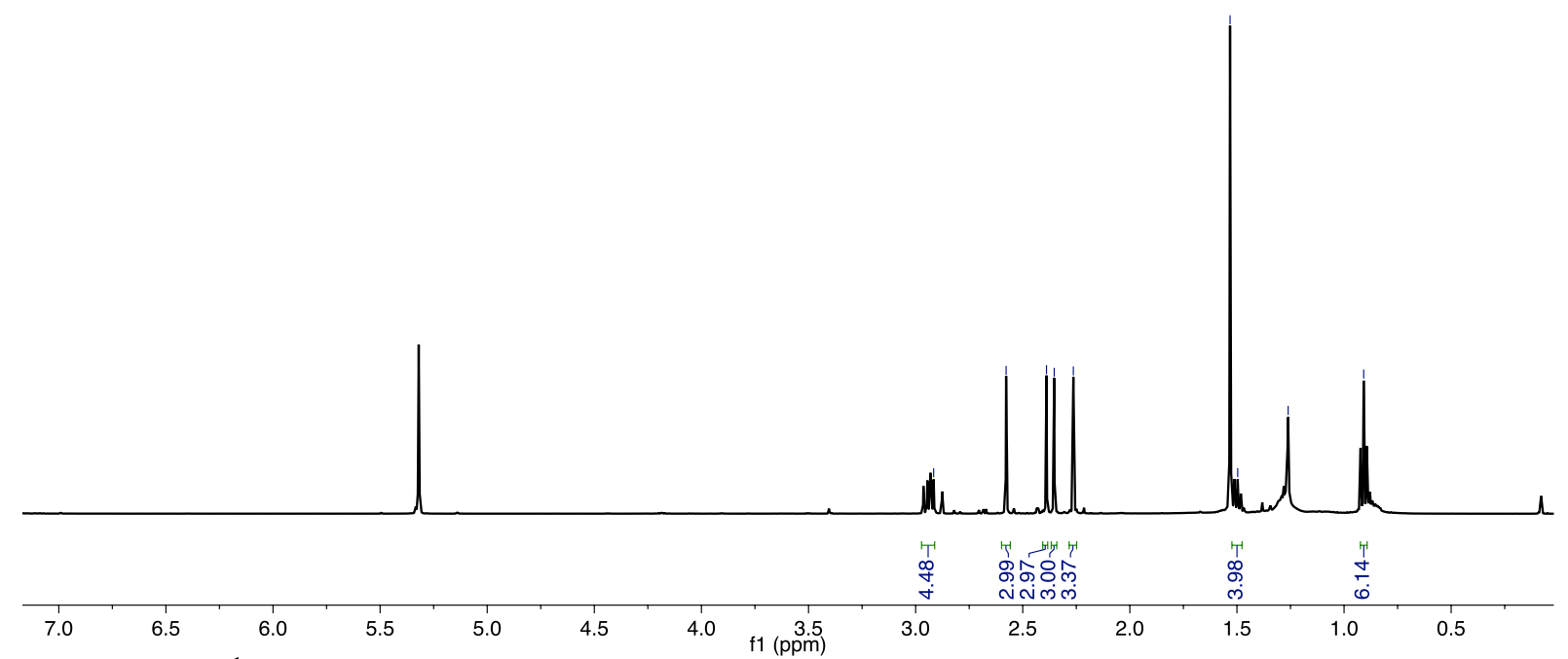

Figure S43. ${ }^{1} \mathrm{H}$ NMR spectrum of 7 in $\mathrm{CD}_{2} \mathrm{Cl}_{2}$.

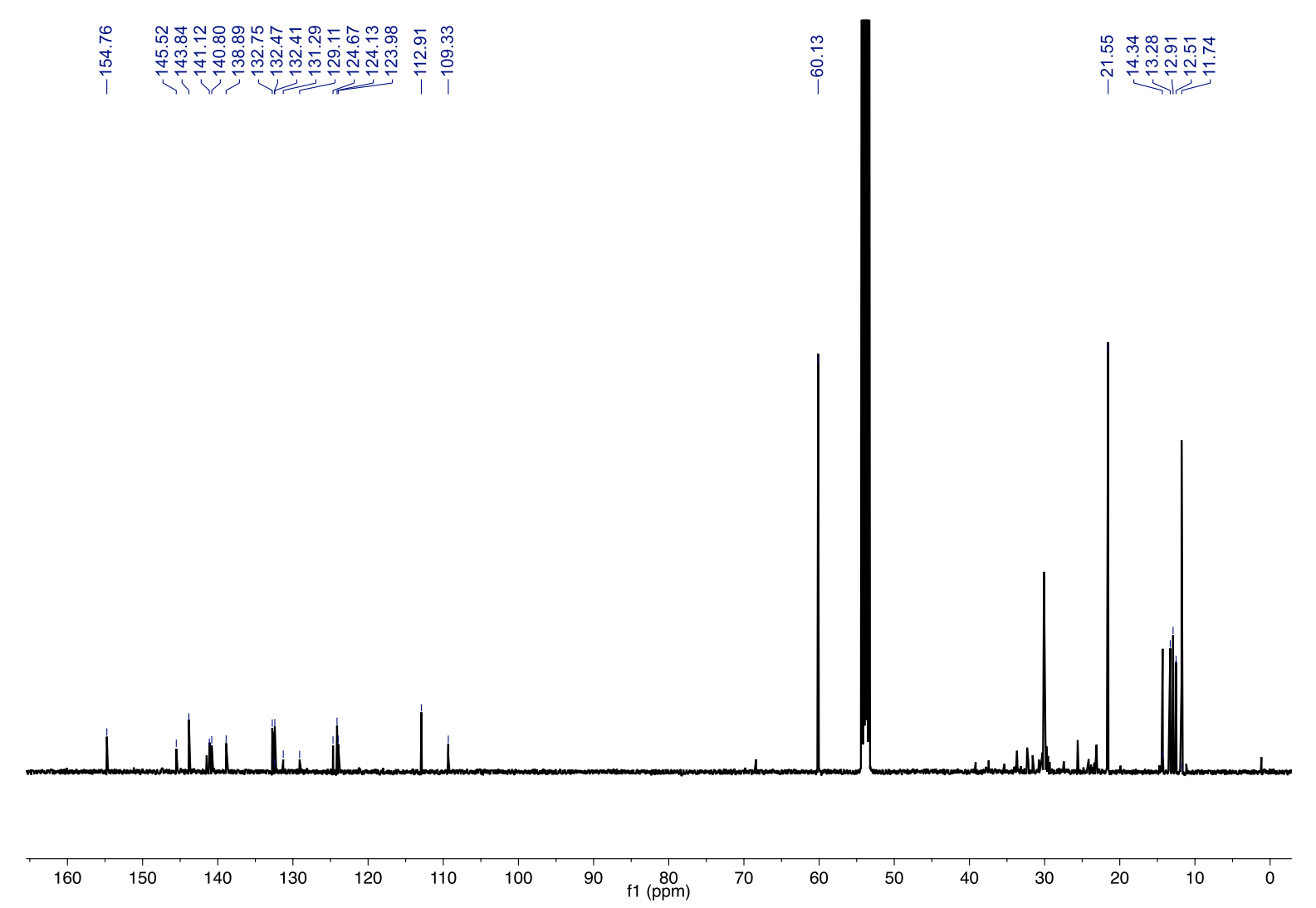

Figure S44. ${ }^{13} \mathrm{C}$ NMR spectrum of 7 in $\mathrm{CD}_{2} \mathrm{Cl}_{2}$. 


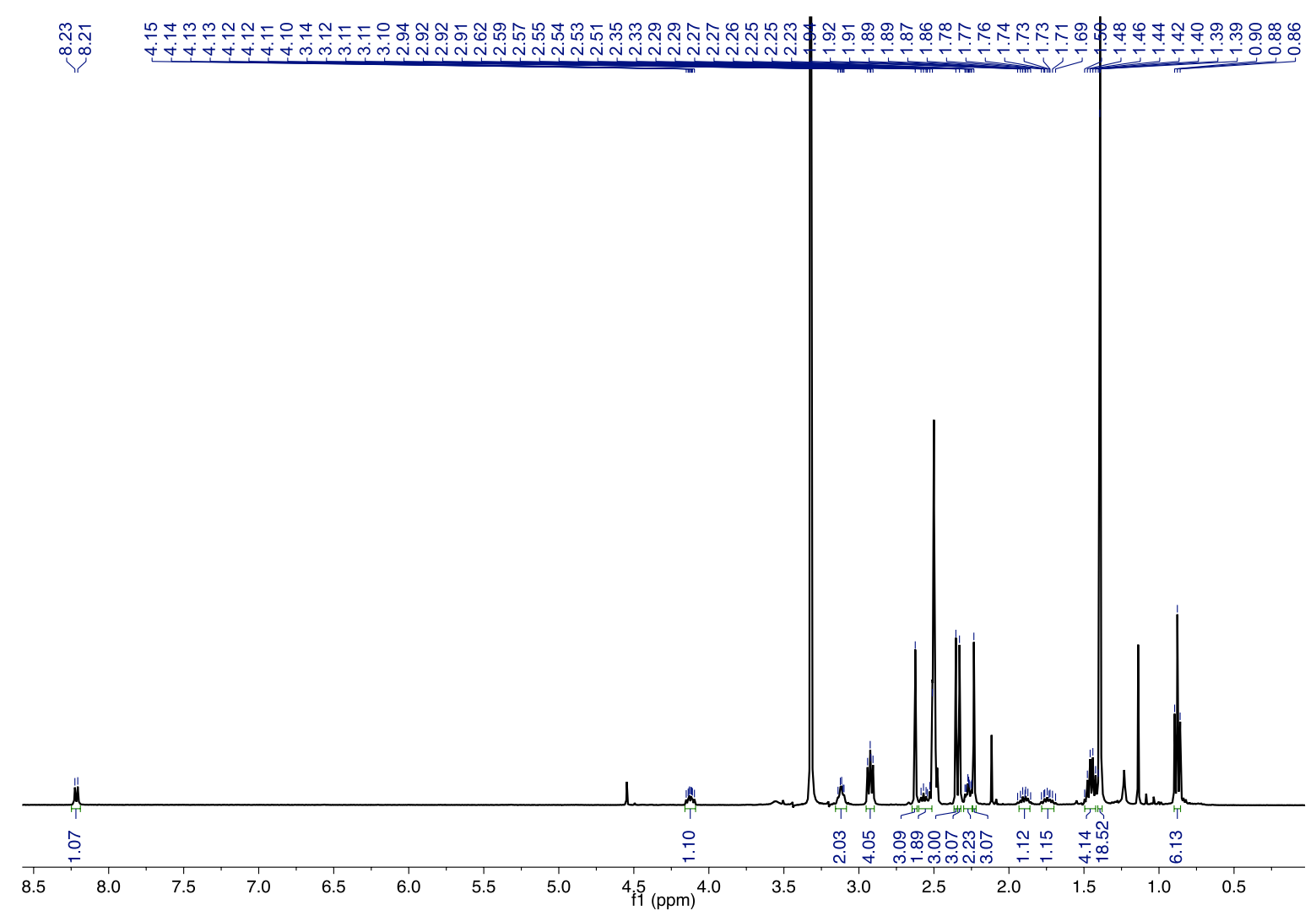

Figure S45. ${ }^{1} \mathrm{H}$ NMR spectrum of 8 in DMSO- $d_{6}$.

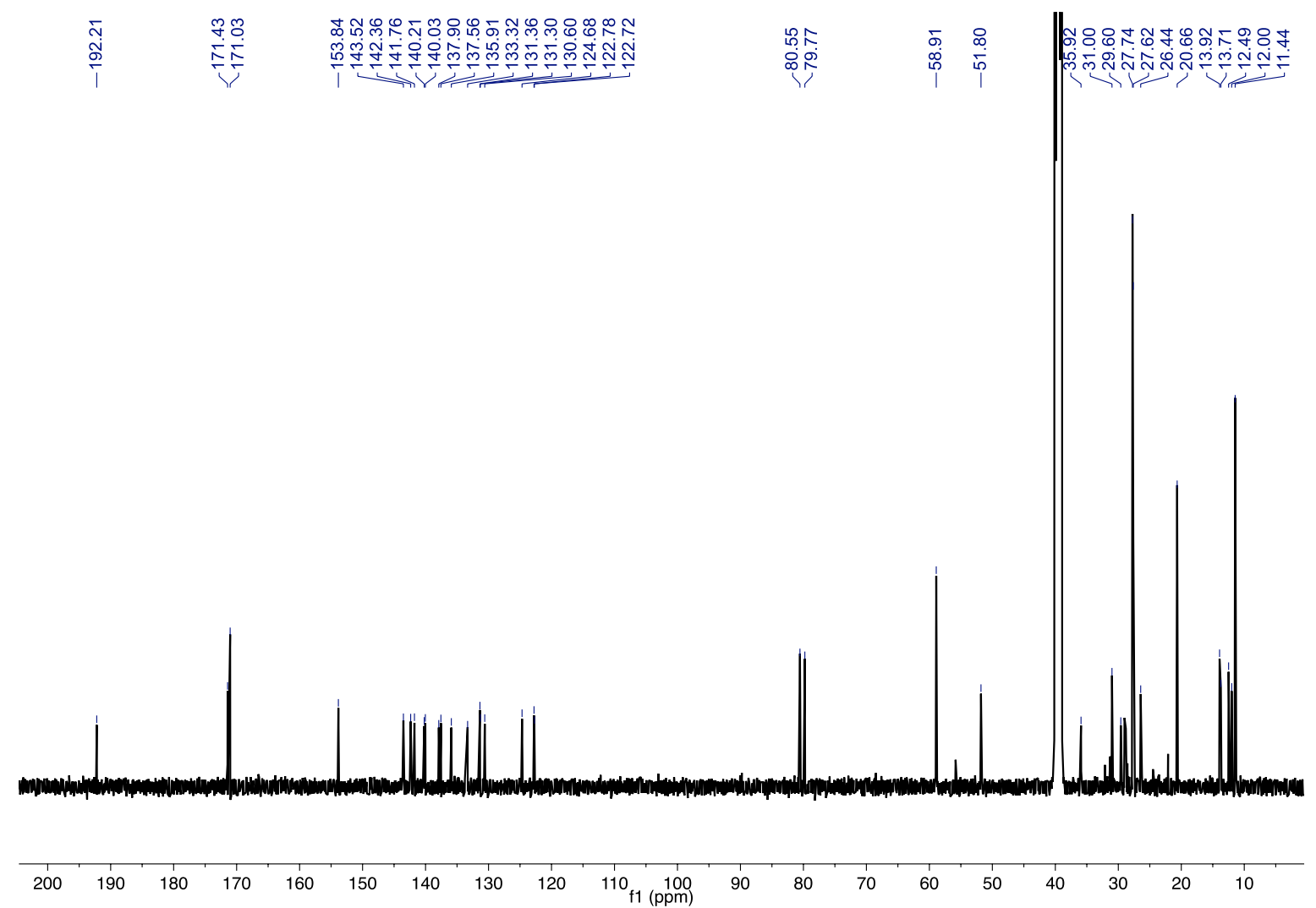

Figure S46. ${ }^{13} \mathrm{C}$ NMR spectrum of 8 in DMSO- $d_{6}$. 


\section{MALDI-TOF MS}
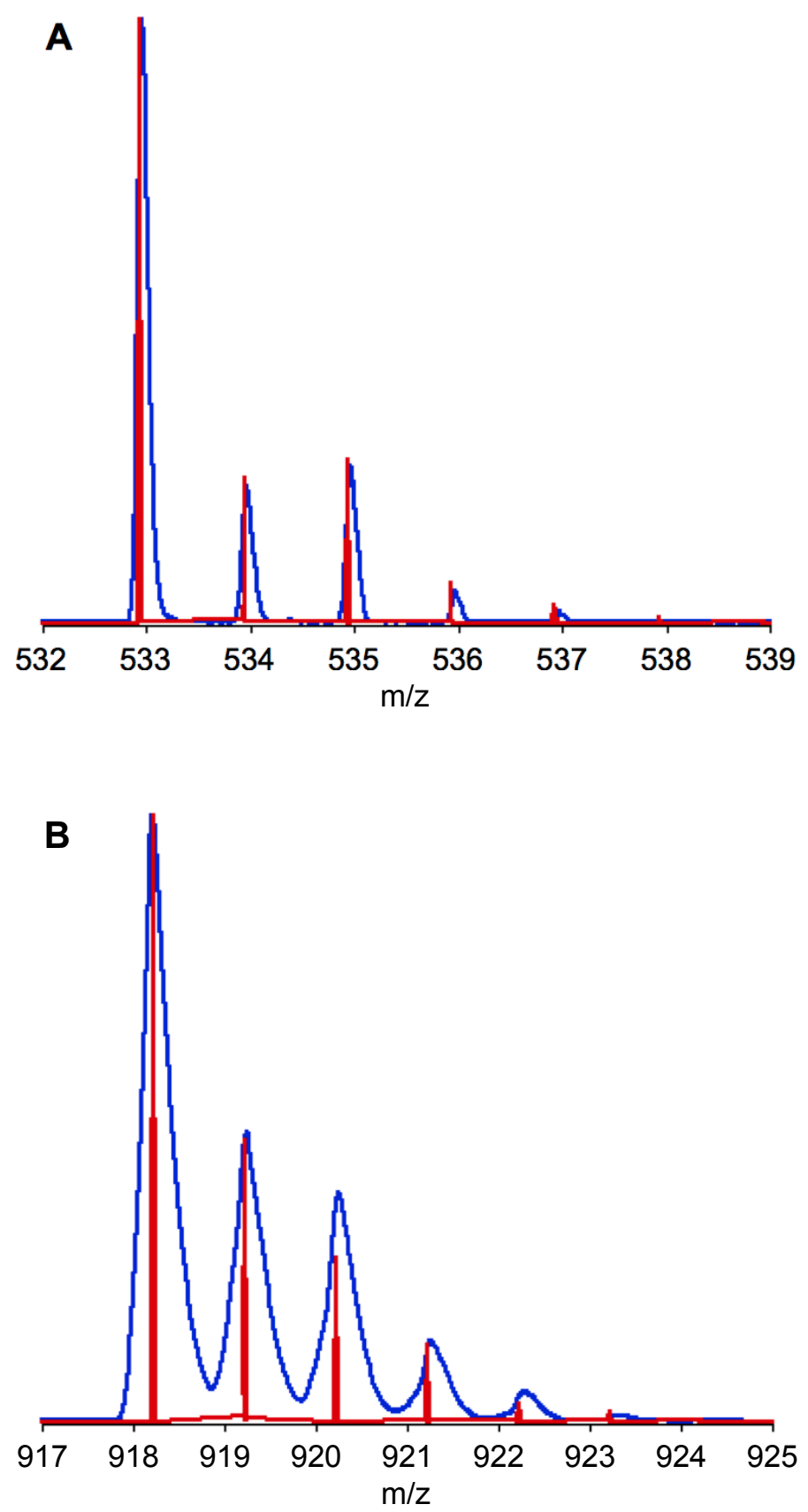

Figure S47. MALDI-TOF MS of compounds 5 (A) and 8 (B). Experimental data (blue) and simulated isotope distributions (red). 